\title{
TOPOLOGICAL APPLICATIONS OF GRADED FROBENIUS $n$-HOMOMORPHISMS
}

\author{
D. V. GUGNIN
}

\begin{abstract}
This paper generalizes the theory of Frobenius $n$-homomorphisms, as expounded by V. M. Buchstaber and E. G. Rees, to graded algebras, and applies the new algebraic technique of graded Frobenius $n$-homomorphisms to two topological problems. The first problem is to find estimates on the cohomological length of the base and of the total space of a wide class of branched coverings of topological spaces, called the Smith-Dold branched coverings. This class of branched coverings contains, in particular, unbranched finite-sheeted coverings and the usual finite-sheeted branched coverings from the theory of smooth manifolds. The second problem concerns a description of cohomology and fundamental groups of $n$-valued topological groups. The main tool there is a generalization of the notion of a graded Hopf algebra, based on the notion of a graded Frobenius $n$-homomorphism.
\end{abstract}

\section{INTRODUCTION}

The goal of this work is threefold. First, we want to generalize the work of V. M. Buchstaber and E. G. Rees on Frobenius $n$-homomorphisms to the case of graded algebras. Secondly, we want to apply the new algebraic techniques to the study of cohomology of branched coverings. Finally, using a generalization, based on the notion of graded $n$-homomorphisms, of the notion of a Hopf algebra, we want to study cohomology and fundamental groups of spaces endowed with a structure of an $n$-valued topological group.

In 1896, Frobenius [22, 23, gave a recursive definition of higher characters of finite groups. In [13, 14, V. M. Buchstaber and E. G. Rees introduced the notion of an $n$ algebra homomorphism and showed that it was completely determined by a recursion analogous to the one of Frobenius. For that reason, those maps were called Frobenius $n$-homomorphisms. For further developments, see [8]-10] and [14, 13.

The definition of an $n$-algebra homomorphism was motivated by the theory of $n$ valued topological groups. $n$-valued formal groups were introduced by V. M. Buchstaber and S. P. Novikov [7] in 1971. Later on, V. M. Buchstaber developed a theory of $n$ valued formal groups and its topological applications. In [6], he discovered an important structure of a 2-valued algebraic group on $S^{2}$. That marked the beginning of a topological theory of $n$-valued groups, which was developed by V. M. Buchstaber and E. G. Rees [12] 14], as well as by A. M. Vershik, A. P. Veselov, A. A. Gaifullin, S. A. Evdokimov, T. E. Panov, I. N. Ponomarenko, A. N. Kholodov, and P. V. Yagodovsky (for a detailed survey, see [5]). The theory of $n$-valued groups, including their representations and actions, has also found applications in dynamical systems [15, 20].

\footnotetext{
2010 Mathematics Subject Classification. Primary 17A42; Secondary 57M12.

Key words and phrases. Graded algebra, graded $n$-homomorphism, Frobenius, Smith-Dold branched covering, cohomological length, $n$-valued topological group.

Supported by the RFFI grants 10-01-92102-YaF-a and 11-01-00694-a, President's Grant for leading scientific schools, Project NSh-5413.2010.1, and the Government Grant 2010-220-01-077, Contract 11.G34.31.0005.
} 
A well-known problem in algebraic topology asks for which classes of continuous maps $f: X \rightarrow Y$ of topological spaces there exists a direct image $f_{!}: H^{*}(X) \rightarrow H^{*}(Y)$ in cohomology such that the composition $f_{!} \circ f^{*}: H^{*}(Y) \rightarrow H^{*}(Y)$ is multiplication by an integer. Various kinds of branched coverings are well known in topology, algebraic geometry, and complex analysis. In 1983, L. Smith [28] introduced the notion of an $n$-fold branched covering, which admitted a direct image in cohomology. In 1986, A. Dold [17 obtained a complete classification of $n$-fold branched coverings (introduced by L. Smith) in terms of actions of finite groups on spaces. Such maps were later called $n$-fold SmithDold branched coverings. In this paper, we study the cohomology of branched coverings of exactly this type. A connection between $n$-fold Smith-Dold branched coverings and (nongraded) $n$-homomorphisms was first established by V. M. Buchstaber and E. G. Rees in 8 .

The following three topologically important classes of continuous maps are known to be $n$-fold Smith-Dold branched coverings:

1) unbranched $n$-fold coverings $f: X \rightarrow Y$;

2) projections $\pi: X \rightarrow X / G$ onto the quotient by an action of a finite group $G$ of order $n$;

3) the usual $n$-fold branched coverings in the theory of smooth manifolds (with branch locus along codimension 2 submanifolds).

This paper consists of three chapters. We now describe the contents of each section and state their main results.

In the first section, we generalize the notion of an $n$-homomorphism to graded algebras. Informally speaking, $n$-homomorphisms are a special class of linear maps between graded associative algebras, which are weakly multiplicative in some special sense. A 1-homomorphism is exactly an algebra homomorphism. Weak multiplicativity for a 2homomorphism $f: A^{*} \rightarrow B^{*}$ between commutative graded algebras means that we can compute the value $f\left(a_{1} a_{2} a_{3}\right)$ of $f$ on the product of any three homogeneous elements $a_{1}, a_{2}, a_{3} \in A^{*}$ as a concrete polynomial in $f\left(a_{1}\right), f\left(a_{2}\right), f\left(a_{3}\right), f\left(a_{1} a_{2}\right), f\left(a_{1} a_{3}\right), f\left(a_{2} a_{3}\right)$. Moreover, the coefficients of that polynomial are rational numbers depending only on the parities of the degrees $\left|a_{1}\right|,\left|a_{2}\right|,\left|a_{3}\right|$.

Recall that the sum $f=f_{1}+f_{2}: A^{*} \rightarrow B^{*}$ of two algebra homomorphisms $f_{i}: A^{*} \rightarrow$ $B^{*}, i=1,2$, is not an algebra homomorphism because the condition $f\left(a_{1} a_{2}\right)=f\left(a_{1}\right) f\left(a_{2}\right)$ $\forall a_{1}, a_{2} \in A^{*}$ does not hold. But it turns out that weak multiplicativity for this class of linear maps does hold. This means that the sum $f=f_{1}+f_{2}$ of two algebra homomorphisms is a 2-homomorphism. Similarly, the sum $f=f_{1}+\cdots+f_{n}: A^{*} \rightarrow B^{*}$ of $n$ algebra homomorphisms is a typical (and the most important for applications) example of an $n$-homomorphism.

In the first section, we define a graded Frobenius recursion (this is the precise definition of weak multiplicativity) and establish basic properties of $n$-homomorphisms. The biggest difficulty in the graded case, as compared with the nongraded one, is the correct definition of the sign in the graded Frobenius recursion, which depends on the parities of the degrees. The main result of that section is Theorem 1.2.3.

Suppose $R^{*}$ is an associative graded ring with identity, $A^{*}$ and $B^{*}$ are graded associative $R^{*}$-algebras with identity, and assume also that $B^{*}$ is commutative.

Theorem 1.2.3. Suppose $A^{*}$ and $B^{*}$ are $R^{*}$-algebras, $B^{*}$ is commutative, $f: A^{*} \rightarrow B^{*}$ is an n-homomorphism and $g: A^{*} \rightarrow B^{*}$ is an m-homomorphism. Then $f+g: A^{*} \rightarrow B^{*}$ is an $(n+m)$-homomorphism.

This theorem implies that the sum $f=f_{1}+\cdots+f_{n}: A^{*} \rightarrow B^{*}$ of $n$ algebra homomorphisms is an $n$-homomorphism. We remark however that, as was shown by the author [24], already in the nongraded commutative case there are $n$-homomorphisms 
$f: A \rightarrow B$ not representable in the form $f=g+h$, where $g: A \rightarrow B$ is a $k$-homomorphism and $h: A \rightarrow B$ is an $(n-k)$-homomorphism, for any $1 \leq k \leq n-1$.

In the second section, we apply the algebraic technique from Section 1 to the study of the cohomology of Smith-Dold branched coverings. Using a result of A. Dold and the classical transfer from group theory, it is not difficult to show that for any $n$-fold Smith-Dold branched covering $f: X \rightarrow Y$ the induced maps $f^{*}: H^{*}(Y ; \mathbb{Q}) \rightarrow H^{*}(X ; \mathbb{Q})$ and $f^{*}: H^{*}\left(Y ; \mathbb{Z}_{p}\right) \rightarrow H^{*}\left(X ; \mathbb{Z}_{p}\right)$ in rational and, respectively, $\mathbb{Z}_{p}, p>n$ cohomology are monomorphisms. The main question, which we partially answer in the second section, is:

For a given $n$ and a fixed algebra $H^{*}(X ; \mathbb{Q})$, how small ("degenerate") could the subalgebra $H^{*}(Y ; \mathbb{Q}) \subset H^{*}(X ; \mathbb{Q})$ (or $\left.H^{*}\left(Y ; \mathbb{Z}_{p}\right) \subset H^{*}\left(X ; \mathbb{Z}_{p}\right), p>n\right)$ be?

It follows from the theory of actions of finite groups and a result of $\mathrm{A}$. Dold that there are direct linear maps $\tau: H^{*}(X ; \mathbb{Q}) \rightarrow H^{*}(Y ; \mathbb{Q})$ and $\tau: H^{*}\left(X ; \mathbb{Z}_{p}\right) \rightarrow H^{*}\left(Y ; \mathbb{Z}_{p}\right)$ in cohomology. It turns out that these maps $\tau$ are $n$-homomorphisms of a special type $(n$ transfers). Let $l(X)$ denote the rational cohomological length of $X$, i.e., the largest integer $m$ such that there are homogeneous elements $a_{1}, a_{2}, \ldots, a_{m} \in H^{* \geq 1}(X ; \mathbb{Q})$ of positive degree such that $a_{1} a_{2} \ldots a_{m} \neq 0$. Similarly, let $l_{p}(X)$ denote the $\mathbb{Z}_{p}$-cohomological length of $X$. Using various properties of $n$-transfer, we have the main result of the second section:

Theorem 2.2.3. Suppose $X$ and $Y$ are locally contractible paracompact spaces and $f: X \rightarrow Y$ is an $n$-fold Smith-Dold branched covering. Then:

$$
l(Y)+1 \geq \frac{l(X)+1}{n}, \quad l_{p}(Y)+1 \geq \frac{l_{p}(X)+1}{n} \quad \forall p>n .
$$

When $n=2$, this bound is sharp.

We remark that this result is somewhat deficient in the sense that it only applies to branched coverings with a relatively small number of sheets. Namely, for $n>\operatorname{dim} X=$ $\operatorname{dim} Y$ (the dimensions of $X$ and $Y$ coincide in all the usual cases, for example, for polyhedra) our estimate becomes trivial, $l(Y) \geq 0$ and $l_{p}(Y) \geq 0 \forall p>n$. In particular, for branched coverings $f: M^{m} \rightarrow N^{m}$ of manifolds, $n$ should not exceed $m$.

In the third section, we study the cohomology and fundamental groups of spaces endowed with a structure of an $n$-valued topological group. An $n$-valued multiplication on a space $X$ is a continuous map $\mu: X \times X \rightarrow \operatorname{Sym}^{n} X$, where $\operatorname{Sym}^{n} X:=X^{n} / S_{n}$ is the $n$-th symmetric power of $X$. An $n$-valued topological group is a Hausdorff space $X$ with base point $e \in X, n$-valued multiplication $\mu: X \times X \rightarrow \operatorname{Sym}^{n} X$, and a continuous map inv: $X \rightarrow X$ (inverse), subject to certain axioms for unit, associativity, and inverse. In particular, the unit axiom is formulated as follows: $\mu(x, e)=\mu(e, x)=[n x] \in \operatorname{Sym}^{n} X$ $\forall x \in X$. A 1-valued group is just a topological group.

It is known that the rational cohomology algebra of a Lie group (or more generally, of its homotopy analog, an $H$-group) is a graded Hopf algebra. In particular, if $X$ is an $H$-group, then $H^{*}(X ; \mathbb{Q})$ is a free commutative algebra on some number of even and odd generators, and is thus isomorphic to the tensor product of an exterior algebra and a polynomial algebra. (Of course, if $X$ is finite-dimensional, there are no even generators.)

In Section 3, we also introduce a notion of a graded $n$-Hopf algebra. The main difference from the usual axioms for a graded Hopf algebra is in the requirement that the diagonal $\Delta: A^{*} \rightarrow A^{*} \otimes A^{*}$ be an $n$-homomorphism. In $\S 3.1$, we prove Theorem 3.1.7, asserting that the rational cohomology algebra $H^{*}(X ; \mathbb{Q})$ of an $n$-valued topological group (and, more generally, of an $n H$-group) is a graded $n$-Hopf algebra. In the special case when the odd-degree cohomology groups $H^{\text {odd }}(X ; \mathbb{Q})$ vanish, this theorem was proved by V. M. Buchstaber and E. G. Rees [13]. Related to this is a theorem of T. E. Panov [27, 
which classifies all four-dimensional simply connected closed manifolds $M^{4}$ such that the rational cohomology algebra $H^{*}\left(M^{4} ; \mathbb{Q}\right)$ admits a structure of a graded 2-Hopf algebra.

Besides $n H$-groups as homotopical analogs of $n$-valued topological groups, in Section 3, we also consider $n H$-monoids (defined only by the axioms of homotopy unit and homotopy associativity) and $n H$-spaces (defined only by the axiom of homotopy unit). A $1 H$-space is just an $H$-space. It is well known that the fundamental group of any $H$-space is abelian, and for any abelian group $A$ there is an $H$-space (or even a loop space) $K(A, 1)=\Omega K(A, 2)$ with fundamental group $A$. In $\S 3.2$, we consider the next, in terms of complexity, question: what are the fundamental groups of $2 \mathrm{H}$-spaces? There we introduce a rather wide class $\mathcal{C}$ of finitely determined groups, which includes, in particular, fundamental groups of compact Riemann surfaces of genus $g \geq 2$. The main result of Section 3 is the following.

Theorem 3.2.4. Suppose $X$ is a connected countable $C W$-complex such that $\operatorname{dim} H^{q}(X ; \mathbb{Q})<\infty \forall q \geq 0$. If $X$ admits a structure of a $2 H$-space, then its fundamental group does not belong to $\mathcal{C}$.

We also give examples of $2 H$-spaces with nonabelian fundamental groups.

\section{THEORY OF GRADED FrobENIUS $n$-HOMOMORPHISMS}

1.1. Graded Frobenius recursion. Henceforth, the base ring will always be a commutative associative graded ring $R^{*}=\bigoplus_{i=0}^{\infty} R^{i}$ with identity $1 \in R^{0}, 1 \neq 0$. An algebra over the base ring will always be a graded associative (but not necessarily commutative) $R^{*}$-algebra $A^{*}$ with identity. We will consider $A^{*}$ as a bimodule over $R^{*}$, i.e., $\lambda a=(-1)^{|\lambda||a|} a \lambda \forall \lambda \in R^{*}, \forall a \in A^{*}$. All maps $f: A^{*} \rightarrow B^{*}$ between two $R^{*}$-algebras will be graded of degree zero and two-sided $R^{*}$-linear, which means:

(1) $f\left(A^{i}\right) \subset B^{i} \quad \forall i \in \mathbb{Z}_{+}$;

(2) $f\left(a_{1}+a_{2}\right)=f\left(a_{1}\right)+f\left(a_{2}\right) \quad \forall a_{1}, a_{2} \in A^{*}$;

(3) $f(\lambda a)=\lambda f(a), f(a \lambda)=f(a) \lambda \quad \forall \lambda \in R^{*}, \forall a \in A^{*}$.

It is not difficult to show that, in the definition of two-sided $R^{*}$-linearity for degree zero maps, it suffices to require linearity only on the left $\left(f(\lambda a)=\lambda f(a) \forall \lambda \in R^{*}, \forall a \in A^{*}\right)$, or linearity only on the right $\left(f(a \lambda)=f(a) \lambda \forall \lambda \in R^{*}, \forall a \in A^{*}\right)$.

In other words, for degree zero maps, one-sided $R^{*}$-linearity implies two-sided $R^{*}$ linearity; so we shall just call such maps $R^{*}$-linear.

Our immediate goal is to define, for any degree zero $R^{*}$-linear map $f: A^{*} \rightarrow B^{*}$ of an $R^{*}$-algebra $A^{*}$ to a commutative $R^{*}$-algebra $B^{*}$ such that $f(a b)=(-1)^{|a||b|} f(b a)$ $\forall a, b \in A^{*}$, and for any natural number $m$, some special degree zero $m$-linear symmetric maps $\Phi_{m}(f): A^{m} \rightarrow B^{*}$.

First, we need some notation. For any $p, q \in \mathbb{N}$, we set $\varepsilon^{p q}=1$ if $p<q$, and $\varepsilon^{p q}=0$ if $p \geq q$. We also set $\varepsilon_{p q}=1$ if $p>q$, and $\varepsilon_{p q}=0$, if $p \leq q$.

Fix some $m \in \mathbb{N}$ and consider an arbitrary permutation $\sigma \in S_{m}$. Represent $\sigma$ as a product of disjoint cycles $\sigma=\gamma_{1} \gamma_{2} \ldots \gamma_{s}, \gamma_{i}=\left(l_{1}^{i} \ldots l_{k_{i}}^{i}\right), 1 \leq i \leq s$, including cycles of length one.

Now we totally order, in some way, the cycles $\gamma_{1}, \ldots, \gamma_{s}$ and, in each cycle $\gamma_{i}, 1 \leq$ $i \leq s$, arbitrarily choose an initial element. Our choices are equivalent to a representation $\sigma=\gamma_{1} \gamma_{2} \ldots \gamma_{s}=\left(l_{1}^{1} \ldots l_{k_{1}}^{1}\right)\left(l_{1}^{2} \ldots l_{k_{2}}^{2}\right) \ldots\left(l_{1}^{s} \ldots l_{k_{s}}^{s}\right)$. The numerical sequence $\left(l_{1}^{1} \ldots l_{k_{1}}^{1} l_{1}^{2} \ldots l_{k_{2}}^{2} \ldots l_{1}^{s} \ldots l_{k_{s}}^{s}\right)$ will be denoted

$$
\left(i_{1}, \ldots, i_{m}\right)=\left(i_{1}, \ldots, i_{k_{1}}, i_{\left(k_{1}+1\right)}, \ldots, i_{\left(k_{1}+k_{2}\right)}, \ldots, i_{\left(k_{1}+k_{2}+\ldots+k_{s-1}+1\right)}, \ldots, i_{m}\right) .
$$

Thus, given the permutation $\sigma$, we have exactly $s ! k_{1} \ldots k_{s}$ ways to obtain a permutation $i=\left(i_{1}, i_{2}, \ldots, i_{m}\right)$ of $\{1,2, \ldots, m\}$. 
Suppose now that $f: A^{*} \rightarrow B^{*}$ is a degree zero $R^{*}$-linear map of $R^{*}$-algebras $\left(B^{*}\right.$ is commutative). We also assume that $f(a b)=(-1)^{|a||b|} f(b a) \forall a, b \in A^{*}$. For any $m \in \mathbb{N}$, any homogeneous elements $a_{1}, \ldots, a_{m} \in A^{*}$, any permutation $\sigma \in S_{m}$, and any permutation

$$
i=\left(i_{1}, \ldots, i_{m}\right)=\left(i_{1}, \ldots, i_{k_{1}}, i_{\left(k_{1}+1\right)}, \ldots, i_{\left(k_{1}+k_{2}\right)}, \ldots, i_{\left(k_{1}+k_{2}+\ldots+k_{s-1}+1\right)}, \ldots, i_{m}\right),
$$

defined by $\sigma$ as above, we can compute

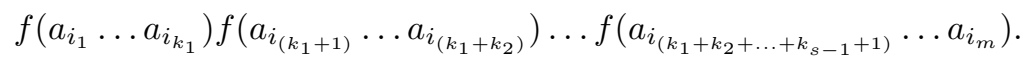

Now we can define maps $\Phi_{m}(f): A^{m} \rightarrow B^{*}$ by an explicit formula.

\section{Definition 1.1.1.}

$$
\begin{aligned}
\Phi_{m}(f)\left(a_{1}, \ldots, a_{m}\right) & =\sum_{\sigma \in S_{m}}(-1)^{\sigma}(-1)^{\sum_{p, q=1}^{m} \varepsilon^{p q} \varepsilon_{i^{-1}(p) i^{-1}(q)}\left|a_{p}\right|\left|a_{q}\right|} \\
& \times f\left(a_{i_{1}} \ldots a_{i_{k_{1}}}\right) f\left(a_{i_{\left(k_{1}+1\right)}} \ldots a_{i_{\left(k_{1}+k_{2}\right)}}\right) \ldots f\left(a_{i_{\left(k_{1}+k_{2}+\ldots+k_{s-1}+1\right)}} \ldots a_{i_{m}}\right) .
\end{aligned}
$$

Before showing that formula $(*)$ is well-defined, we shall prove an auxiliary result about the sign.

Lemma 1.1.2. Suppose $A^{*}$ is a commutative $R^{*}$-algebra, $a_{1}, \ldots, a_{m} \in A^{*}$ are homogeneous elements, and $\sigma$ is a permutation from $S_{m}$. Then:

$$
a_{\sigma(1)} a_{\sigma(2)} \ldots a_{\sigma(m)}=(-1)^{\sum_{p, q=1}^{m} \varepsilon^{p q} \varepsilon_{\sigma^{-1}(p) \sigma^{-1}(q)}\left|a_{p}\right|\left|a_{q}\right|} a_{1} a_{2} \ldots a_{m} .
$$

Proof. What we need to show is that the expression

$$
(-1)^{\sum_{p, q=1}^{m} \varepsilon^{p q} \varepsilon_{\sigma^{-1}(p) \sigma^{-1}(q)}\left|a_{p}\right|\left|a_{q}\right|} a_{\sigma(1)} a_{\sigma(2)} \ldots a_{\sigma(m)},
$$

which we denote by $F_{\sigma}$, is a constant, not depending on $\sigma \in S_{m}$. Then we will have $F_{\sigma}=F_{e}=a_{1} a_{2} \ldots a_{m} \forall \sigma \in S_{m}$.

It is helpful to introduce the following graph $\Gamma_{m}$. The vertices of $\Gamma_{m}$ are all permutations $\sigma \in S_{m}$. Each vertex $\sigma$ of $\Gamma_{m}$ is the source of exactly $m-1$ oriented edges $\delta_{1}, \ldots, \delta_{m-1}$. More precisely, the edge $\delta_{i}, 1 \leq i \leq m-1$, connects $\sigma$ with the following permutation $\tau=\delta_{i}(\sigma): \tau(k)=\sigma(k), 1 \leq k \leq m, k \neq i, i+1, \tau(i)=\sigma(i+1)$, $\tau(i+1)=\sigma(i)$. In other words, $\tau=\delta_{i}(\sigma), 1 \leq i \leq m-1$, differs from $\sigma$ by the transposition of the adjacent indices $\sigma(i)$ and $\sigma(i+1)$. It is clear that if $\tau=\delta_{i}(\sigma)$, then $\sigma=\delta_{i}(\tau)$. Since any permutation $\sigma$ can be obtained from the identity permutation $e$ by a sequence of transformations $\delta_{i}, 1 \leq i \leq m-1$, we see that $\Gamma_{m}$ is strongly connected; i.e., there is a directed path from any vertex to any other vertex. Thus, to prove that $F_{\sigma}=F_{e}$, $\forall \sigma \in S_{m}$ it suffices to show that $F_{\sigma}=F_{\tau}$ if $\tau=\delta_{i}(\sigma), 1 \leq i \leq m-1$.

Now we take a closer look at the sign $(-1)^{\sum_{p, q=1}^{m} \varepsilon^{p q} \varepsilon_{\sigma^{-1}(p) \sigma^{-1}(q)}\left|a_{p}\right|\left|a_{q}\right|}$, which we denote for brevity by $(-1)^{\varepsilon_{\sigma}\left(\left|a_{1}\right|, \ldots,\left|a_{m}\right|\right)}$. It is not difficult to see that, among the $C_{m}^{2}$ summands $\left|a_{p}\right|\left|a_{q}\right|, 1 \leq p<q \leq m$, the ones which enter the sum $\varepsilon_{\sigma}\left(\left|a_{1}\right|, \ldots,\left|a_{m}\right|\right)$ with a nonzero coefficient (the coefficient in that case equals +1 ) are those for which the term $a_{p}$ is to the right of $a_{q}$ in $a_{\sigma(1)} a_{\sigma(2)} \ldots a_{\sigma(m)}$. We need to establish the following equality:

$$
(-1)^{\varepsilon_{\sigma}\left(\left|a_{1}\right|, \ldots,\left|a_{m}\right|\right)} a_{\sigma(1)} a_{\sigma(2)} \ldots a_{\sigma(m)}=(-1)^{\varepsilon_{\tau}\left(\left|a_{1}\right|, \ldots,\left|a_{m}\right|\right)} a_{\tau(1)} a_{\tau(2)} \ldots a_{\tau(m)} .
$$

It can be rewritten as follows:

$$
\begin{gathered}
(-1)^{\varepsilon_{\sigma}\left(\left|a_{1}\right|, \ldots,\left|a_{m}\right|\right)+\varepsilon_{\tau}\left(\left|a_{1}\right|, \ldots,\left|a_{m}\right|\right)} a_{\sigma(1)} a_{\sigma(2)} \ldots a_{\sigma(m)} \\
=a_{\sigma(1)} \ldots a_{\sigma(i-1)}\left(a_{\sigma(i+1)} a_{\sigma(i)}\right) a_{\sigma(i+2)} \ldots a_{\sigma(m)} ; \\
(-1)^{\varepsilon_{\sigma}\left(\left|a_{1}\right|, \ldots,\left|a_{m}\right|\right)+\varepsilon_{\tau}\left(\left|a_{1}\right|, \ldots,\left|a_{m}\right|\right)} a_{\sigma(1)} a_{\sigma(2)} \ldots a_{\sigma(m)}=(-1)^{\left|a_{\sigma(i)}\right|\left|a_{\sigma(i+1)}\right|} a_{\sigma(1)} a_{\sigma(2)} \ldots a_{\sigma(m)} .
\end{gathered}
$$


Thus, for our purposes, it suffices to show that

$$
(-1)^{\varepsilon_{\sigma}\left(\left|a_{1}\right|, \ldots,\left|a_{m}\right|\right)+\varepsilon_{\tau}\left(\left|a_{1}\right|, \ldots,\left|a_{m}\right|\right)}=(-1)^{\left|a_{\sigma(i)}\right|\left|a_{\sigma(i+1)}\right|} .
$$

To this end, we start with the equality

$$
a_{\tau(1)} a_{\tau(2)} \ldots a_{\tau(m)}=a_{\sigma(1)} \ldots a_{\sigma(i-1)}\left(a_{\sigma(i+1)} a_{\sigma(i)}\right) a_{\sigma(i+2)} \ldots a_{\sigma(m)} .
$$

Now look at how the summand $\left|a_{p}\right|\left|a_{q}\right|, 1 \leq p<q \leq m$, enters $\varepsilon_{\sigma}\left(\left|a_{1}\right|, \ldots,\left|a_{m}\right|\right)+$ $\varepsilon_{\tau}\left(\left|a_{1}\right|, \ldots,\left|a_{m}\right|\right)$. We can have the following cases:

( $\alpha)\{p, q\} \cap\{\sigma(i), \sigma(i+1)\}=\varnothing$;

( $\beta)\{p, q\} \cap\{\sigma(i), \sigma(i+1)\}=\{p\}$ or $\{q\}$;

$(\gamma)\{p, q\}=\{\sigma(i), \sigma(i+1)\}$.

In the cases $(\alpha)$ and $(\beta)$, as is easily seen, the summand $\left|a_{p}\right|\left|a_{q}\right|$ enters $\varepsilon_{\sigma}\left(\left|a_{1}\right|, \ldots,\left|a_{m}\right|\right)$ and $\varepsilon_{\tau}\left(\left|a_{1}\right|, \ldots,\left|a_{m}\right|\right)$ with the same coefficient and, therefore, contributes nothing to the sign in question. The case $(\gamma)$ breaks into two subcases:

$(\gamma 1) p=\sigma(i)<q=\sigma(i+1)$.

Here $\left|a_{p}\right|\left|a_{q}\right|=\left|a_{\sigma(i)}\right|\left|a_{\sigma(i+1)}\right|$ enters only $\varepsilon_{\tau}\left(\left|a_{1}\right|, \ldots,\left|a_{m}\right|\right)$ (with coefficient 1). Therefore, (3) holds.

$(\gamma 2) p=\sigma(i+1)<q=\sigma(i)$.

In this subcase, $\left|a_{p}\right|\left|a_{q}\right|=\left|a_{\sigma(i)}\right|\left|a_{\sigma(i+1)}\right|$ enters only $\varepsilon_{\sigma}\left(\left|a_{1}\right|, \ldots,\left|a_{m}\right|\right)$ (with coefficient 1 ). Therefore, (3) holds again.

The lemma is proved.

Proposition 1.1.3. For any $m \geq 1$, formula $(*)$ of Definition 1.1 .1 gives rise to a well-defined degree zero $R^{*}$-linear symmetric map $\Phi_{m}(f): A^{\otimes m} \rightarrow B^{*}$.

Proof. Well-definedness. We want to show that, for a fixed permutation $\sigma \in S_{m}$, the expression

$$
\begin{aligned}
(-1)^{\sum_{p, q=1}^{m} \varepsilon^{p q} \varepsilon_{i^{-1}(p) i-1(q)}\left|a_{p} \| a_{q}\right|} & \\
& \times f\left(a_{i_{1}} \ldots a_{i_{k_{1}}}\right) f\left(a_{i_{\left(k_{1}+1\right)}} \ldots a_{i_{\left(k_{1}+k_{2}\right)}}\right) \ldots f\left(a_{i_{\left(k_{1}+k_{2}+\ldots+k_{s-1}+1\right)} \ldots a_{i_{m}}}\right)
\end{aligned}
$$

does not depend on the choice of the permutation $i=\left(i_{1}, \ldots, i_{m}\right) \in S_{m}$. Denote $(-1)^{\sum_{p, q=1}^{m} \varepsilon^{p q} \varepsilon_{i^{-1}(p) i^{-1}(q)}\left|a_{p}\right|\left|a_{q}\right|}$ by $(-1)^{\varepsilon_{i}\left(\left|a_{1}\right|, \ldots,\left|a_{m}\right|\right)}$, and the whole expression (1) by $F_{\sigma, i}(f)\left(a_{1}, \ldots, a_{m}\right)$. Now fix some permutation $i^{0}=\left(i_{1}^{0}, \ldots, i_{m}^{0}\right) \in S_{m}$ corresponding to $\sigma \in S_{m}$, i.e.,

$$
\begin{gathered}
\sigma=\gamma_{1} \gamma_{2} \ldots \gamma_{s}, \quad \gamma_{j}=\left(l_{1}^{j} \ldots l_{k_{j}}^{j}\right), 1 \leq j \leq s, \\
\left(l_{1}^{1}, \ldots, l_{k_{1}}^{1}, l_{1}^{2}, \ldots, l_{k_{2}}^{2}, \ldots, l_{1}^{s}, \ldots, l_{k_{s}}^{s}\right) \\
=\left(i_{1}^{0}, \ldots, i_{k_{1}}^{0}, i_{\left(k_{1}+1\right)}^{0}, \ldots, i_{\left(k_{1}+k_{2}\right)}^{0}, \ldots, i_{\left(k_{1}+k_{2}+\ldots+k_{s-1}+1\right)}^{0}, \ldots, i_{m}^{0}\right) .
\end{gathered}
$$

Then, beginning with $i^{0}$, we can reach any other permutation $i=\left(i_{1}, \ldots, i_{m}\right) \in S_{m}$ corresponding to $\sigma$, by a sequence of the elementary steps described below. If we could show that (1) is preserved at each step, then it is independent of the choice of permutation $i$, which would show that it is well-defined.

STEP 1 . For some cycle $\gamma_{j}, 1 \leq j \leq s$, we replace the initial element $l_{1}^{j}$ by $l_{2}^{j}$. Now $\gamma_{j}$ rewrites as $\gamma_{j}=\left(l_{2}^{j} l_{3}^{j} \ldots l_{k_{j}}^{j} l_{1}^{j}\right)$. We want to show that $(1)$ remains the same. In that 
formula, besides the sign, only one factor changes

$$
\begin{aligned}
& f\left(a_{i_{\left(k_{1}+\ldots+k_{j-1}+1\right)}^{0}} \ldots a_{i_{\left(k_{1}+\ldots+k_{j}\right)}^{0}}\right) \mapsto f\left(a_{\left.i_{\left(k_{1}+\ldots+k_{j-1}+1\right)} \ldots a_{i_{\left(k_{1}+\ldots+k_{j}\right)}}\right)}\right. \\
& =f\left(a_{i_{\left(k_{1}+\ldots+k_{j-1}+2\right)}^{0}} \cdots a_{i_{\left(k_{1}+\ldots+k_{j}\right)}^{0}} a_{i_{\left(k_{1}+\ldots+k_{j-1}+1\right)}^{0}}\right) \\
& =(-1)^{\left|a_{i_{\left(k_{1}+\ldots+k_{j-1}+1\right)}^{0}}\right| \sum_{r=2}^{k_{j}}\left|a_{i_{\left(k_{1}+\ldots+k_{j-1}+r\right)}^{0}}\right|} f\left(a_{i_{\left(k_{1}+\ldots+k_{j-1}+1\right)}^{0}} \ldots a_{i_{\left(k_{1}+\ldots+k_{j}\right)}^{0}}\right) \text {. }
\end{aligned}
$$

Thus, the new expression and the old one differ only by sign:

$$
\begin{aligned}
F_{\sigma, i}(f)\left(a_{1}, \ldots, a_{m}\right) & =(-1)^{\varepsilon_{i}\left(\left|a_{1}\right|, \ldots,\left|a_{m}\right|\right)}(-1)^{\varepsilon_{i^{0}}\left(\left|a_{1}\right|, \ldots,\left|a_{m}\right|\right)} \\
& \times(-1)^{\left|a_{i_{\left(k_{1}+\ldots+k_{j-1}+1\right)}}\right| \sum_{r=2}^{k_{j}}\left|a_{i_{\left(k_{1}+\ldots+k_{j-1}+r\right)}}\right|} F_{\sigma, i^{0}}(f)\left(a_{1}, \ldots, a_{m}\right) .
\end{aligned}
$$

It remains to show that the resulting sign equals +1 :

(2)

$$
(-1)^{\varepsilon_{i}\left(\left|a_{1}\right|, \ldots,\left|a_{m}\right|\right)}(-1)^{\varepsilon_{i^{0}}\left(\left|a_{1}\right|, \ldots,\left|a_{m}\right|\right)}(-1)^{\left|a_{i_{\left(k_{1}+\ldots+k_{j-1}+1\right)}}\right| \sum_{r=2}^{k_{j}}\left|a_{i_{\left(k_{1}+\ldots+k_{j-1}+r\right)}}\right|}=1 .
$$

Let $F A\left(a_{1}^{\prime}, \ldots, a_{m}^{\prime}\right)$ be a free commutative graded $\mathbb{Q}$-algebra in formal variables $a_{1}^{\prime}, \ldots, a_{m}^{\prime},\left|a_{i}^{\prime}\right|=\left|a_{i}\right|, 1 \leq i \leq m$. By Lemma 1.1.2, we have

$$
\begin{gathered}
(-1)^{\varepsilon_{i}\left(\left|a_{1}\right|, \ldots,\left|a_{m}\right|\right)} a_{i(1)}^{\prime} \ldots a_{i(m)}^{\prime}=a_{1}^{\prime} \ldots a_{m}^{\prime} ; \\
(-1)^{\varepsilon_{i^{0}}\left(\left|a_{1}\right|, \ldots,\left|a_{m}\right|\right)} a_{i^{0}(1)}^{\prime} \ldots a_{i^{0}(m)}^{\prime}=a_{1}^{\prime} \ldots a_{m}^{\prime} .
\end{gathered}
$$

Therefore

$$
a_{i(1)}^{\prime} \ldots a_{i(m)}^{\prime}=(-1)^{\varepsilon_{i}\left(\left|a_{1}\right|, \ldots,\left|a_{m}\right|\right)}(-1)^{\varepsilon_{i^{0}}\left(\left|a_{1}\right|, \ldots,\left|a_{m}\right|\right)} a_{i^{0}(1)}^{\prime} \ldots a_{i^{0}(m)}^{\prime} .
$$

Since

$$
\begin{aligned}
& a_{i_{\left(k_{1}+\ldots+k_{j-1}+1\right)}^{\prime}}^{\prime} \ldots a_{i_{\left(k_{1}+\ldots+k_{j-1}+k_{j}\right)}^{\prime}}^{\prime}=a_{i_{\left(k_{1}+\ldots+k_{j-1}+2\right)}^{0}}^{\prime} \ldots a_{i_{\left(k_{1}+\ldots+k_{j-1}+k_{j}\right)}^{\prime}}^{\prime} a_{i_{\left(k_{1}+\ldots+k_{j-1}+1\right)}^{\prime}}^{\prime}
\end{aligned}
$$

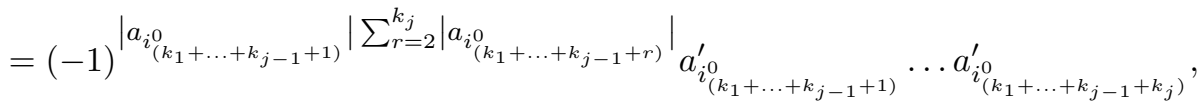

we also have

$$
a_{i(1)}^{\prime} \ldots a_{i(m)}^{\prime}=(-1)^{\left|a_{i_{\left(k_{1}+\ldots+k_{j-1}+1\right)}^{0}}\right| \sum_{r=2}^{k_{j}}\left|a_{i_{\left(k_{1}+\ldots+k_{j-1}+r\right)}}\right|} a_{i^{0}(1)}^{\prime} \ldots a_{i^{0}(m)}^{\prime} .
$$

Combining this formula with (3), we have

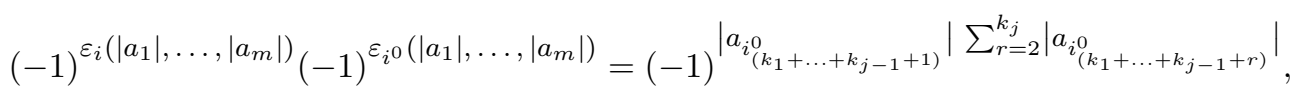

whence formula (2). This completes Step 1.

STEP 2. For some $j, 1 \leq j \leq s-1$, we interchange $\gamma_{j}$ and $\gamma_{j+1}$, without changing their initial elements $l_{1}^{j}$ and $l_{1}^{j+1}$. It is clear that formula (1) will then change the initial sign and that, in the product, the $j$-th and the $(j+1)$-st factors will get transposed; this is equivalent to multiplication by

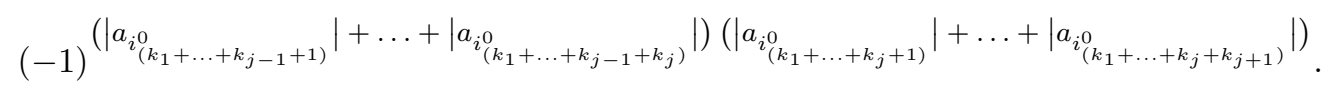


Thus,

(4) $F_{\sigma, i}(f)\left(a_{1}, \ldots, a_{m}\right)=(-1)^{\varepsilon_{i}\left(\left|a_{1}\right|, \ldots,\left|a_{m}\right|\right)}(-1)^{\varepsilon_{i 0}\left(\left|a_{1}\right|, \ldots,\left|a_{m}\right|\right)}$

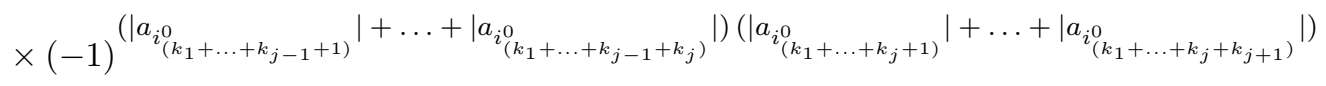

$$
\begin{aligned}
& \times F_{\sigma, i^{0}}(f)\left(a_{1}, \ldots, a_{m}\right) .
\end{aligned}
$$

Once again, let $F A\left(a_{1}^{\prime}, \ldots, a_{m}^{\prime}\right)$ be a free commutative graded $\mathbb{Q}$-algebra in formal variables $a_{1}^{\prime}, \ldots, a_{m}^{\prime},\left|a_{i}^{\prime}\right|=\left|a_{i}\right|, 1 \leq i \leq m$. As before, we have

$$
a_{i(1)}^{\prime} \ldots a_{i(m)}^{\prime}=(-1)^{\varepsilon_{i}\left(\left|a_{1}\right|, \ldots,\left|a_{m}\right|\right)}(-1)^{\varepsilon_{i^{0}}\left(\left|a_{1}\right|, \ldots,\left|a_{m}\right|\right)} a_{i^{0}(1)}^{\prime} \ldots a_{i^{0}(m)}^{\prime} .
$$

It is not difficult to check that the permutation $(i(1), \ldots, i(m))$ is obtained from $\left(i^{0}(1), \ldots, i^{0}(m)\right)$ by setting:

$$
\left\{\begin{array}{l}
i(s)=i^{0}(s), \quad s \leq k_{1}+\ldots+k_{j-1}, \quad s \geq k_{1}+\ldots+k_{j+1}+1, \\
i\left(k_{1}+\ldots+k_{j-1}+t\right)=i^{0}\left(k_{1}+\ldots+k_{j-1}+k_{j}+t\right), \quad 1 \leq t \leq k_{j+1}, \\
i\left(k_{1}+\ldots+k_{j-1}+k_{j+1}+t\right)=i^{0}\left(k_{1}+\ldots+k_{j-1}+t\right), \quad 1 \leq t \leq k_{j} .
\end{array}\right.
$$

It now follows that

$$
\begin{aligned}
& a_{i(1)}^{\prime} \ldots a_{i(m)}^{\prime}
\end{aligned}
$$

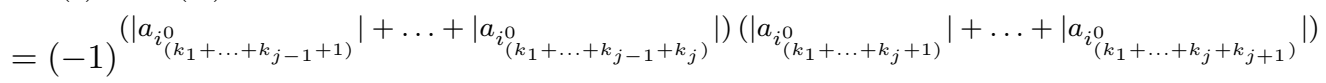

$$
\begin{aligned}
& \times a_{i^{0}(1)}^{\prime} \ldots a_{i^{0}(m)}^{\prime} .
\end{aligned}
$$

Combining this with an earlier formula for $a_{i(1)}^{\prime} \ldots a_{i(m)}^{\prime}$, we have that the sign

$$
\begin{aligned}
& (-1)^{\varepsilon_{i}\left(\left|a_{1}\right|, \ldots,\left|a_{m}\right|\right)}(-1)^{\varepsilon_{i}\left(\left|a_{1}\right|, \ldots,\left|a_{m}\right|\right)}
\end{aligned}
$$

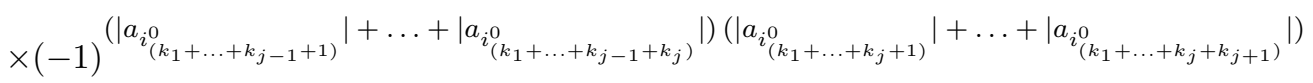

in formula (4) equals +1 . This completes Step 2 and proves the well-definedness of formula (1).

$R^{*}$-linearity. The definition of the map $\Phi_{m}(f): A^{m} \rightarrow B^{*}$ shows that its degree equals zero. The additivity of $\Phi_{m}(f)\left(a_{1}, \ldots, a_{m}\right)$ in each argument is also obvious. Now let us check the left homogeneity in the $s$-th argument, $1 \leq s \leq m$ :

$$
\Phi_{m}(f)\left(a_{1}, \ldots, a_{s-1}, \lambda a_{s}, a_{s+1}, \ldots, a_{m}\right)=(-1)^{|\lambda|\left(\left|a_{1}\right|+\ldots+\left|a_{s-1}\right|\right)} \lambda \Phi_{m}(f)\left(a_{1}, \ldots, a_{m}\right)
$$

$$
\forall \lambda \in R^{*}, \forall a_{1}, \ldots, a_{m} \in A^{*} .
$$

Since the degree of $\Phi_{m}(f): A^{m} \rightarrow B^{*}$ equals zero, this would imply the right (and hence two-sided) homogeneity. We have

$$
\Phi_{m}(f)\left(a_{1}, \ldots, a_{s-1}, \lambda a_{s}, a_{s+1}, \ldots, a_{m}\right)=\sum_{\sigma \in S_{m}}(-1)^{\sigma} F_{\sigma, i}(f)\left(a_{1}, \ldots, \lambda a_{s}, \ldots, a_{m}\right) .
$$

Now fix some $\sigma \in S_{m}$ and choose a permutation $i=(i(1), \ldots, i(m))$ corresponding to $\sigma$ such that $i(1)=s$. Then

$$
\begin{aligned}
& F_{\sigma, i}(f)\left(a_{1}, \ldots, \lambda a_{s}, \ldots, a_{m}\right)=(-1)^{\varepsilon_{i}\left(\left|a_{1}\right|, \ldots,\left|\lambda a_{s}\right|, \ldots,\left|a_{m}\right|\right)} f\left(\lambda a_{s} a_{i_{2}} \ldots a_{i_{k_{1}}}\right) \\
& \times f\left(a_{i_{\left(k_{1}+1\right)}} \ldots a_{i_{\left(k_{1}+k_{2}\right)}}\right) \ldots f\left(a_{i_{\left(k_{1}+\ldots+k_{s-1}+1\right)}} \ldots a_{i_{m}}\right) \\
& =\lambda(-1)^{\varepsilon_{i}\left(\left|a_{1}\right|, \ldots,\left|\lambda a_{s}\right|, \ldots,\left|a_{m}\right|\right)+\varepsilon_{i}\left(\left|a_{1}\right|, \ldots,\left|a_{s}\right|, \ldots,\left|a_{m}\right|\right)} F_{\sigma, i}(f)\left(a_{1}, \ldots, a_{m}\right) \text {. }
\end{aligned}
$$


It remains to compute the sign

$$
(-1)^{\varepsilon_{i}\left(\left|a_{1}\right|, \ldots,\left|\lambda a_{s}\right|, \ldots,\left|a_{m}\right|\right)+\varepsilon_{i}\left(\left|a_{1}\right|, \ldots,\left|a_{s}\right|, \ldots,\left|a_{m}\right|\right) .}
$$

Let $F A\left(\lambda^{\prime}, a_{1}^{\prime}, \ldots, a_{m}^{\prime}\right)$ be a free commutative graded $\mathbb{Q}$-algebra in formal variables $\lambda^{\prime}, a_{1}^{\prime}, \ldots, a_{m}^{\prime},\left|\lambda^{\prime}\right|=|\lambda|,\left|a_{i}^{\prime}\right|=\left|a_{i}\right|, 1 \leq i \leq m$. Then, by Lemma 1.1.2, we have

$$
a_{s}^{\prime} a_{i(2)}^{\prime} \ldots a_{i(m)}^{\prime}=(-1)^{\varepsilon_{i}\left(\left|a_{1}\right|, \ldots,\left|a_{m}\right|\right)} a_{1}^{\prime} \ldots a_{s}^{\prime} \ldots a_{m}^{\prime},
$$

$$
\begin{aligned}
\left(\lambda^{\prime} a_{s}^{\prime}\right) a_{i(2)}^{\prime} \ldots a_{i(m)}^{\prime} & =(-1)^{\varepsilon_{i}\left(\left|a_{1}\right|, \ldots,\left|\lambda a_{s}\right|, \ldots,\left|a_{m}\right|\right)} a_{1}^{\prime} \ldots \lambda^{\prime} a_{s}^{\prime} \ldots a_{m}^{\prime} \\
& =(-1)^{\varepsilon_{i}\left(\left|a_{1}\right|, \ldots,\left|\lambda a_{s}\right|, \ldots,\left|a_{m}\right|\right)}(-1)^{|\lambda|\left|a_{1}\right|+\ldots+|\lambda|\left|a_{s-1}\right|} \lambda^{\prime} a_{1}^{\prime} \ldots a_{s}^{\prime} \ldots a_{m}^{\prime} .
\end{aligned}
$$

Multiplying formula (6) on the left by $\lambda^{\prime}$, we have

$$
\left(\lambda^{\prime} a_{s}^{\prime}\right) a_{i(2)}^{\prime} \ldots a_{i(m)}^{\prime}=(-1)^{\varepsilon_{i}\left(\left|a_{1}\right|, \ldots,\left|a_{m}\right|\right)} \lambda^{\prime} a_{1}^{\prime} \ldots a_{s}^{\prime} \ldots a_{m}^{\prime} .
$$

Comparing (7) and (8), we have that the sign in question

$$
(-1)^{\varepsilon_{i}\left(\left|a_{1}\right|, \ldots,\left|\lambda a_{s}\right|, \ldots,\left|a_{m}\right|\right)+\varepsilon_{i}\left(\left|a_{1}\right|, \ldots,\left|a_{s}\right|, \ldots,\left|a_{m}\right|\right)}
$$

equals

$$
(-1)^{|\lambda|\left|a_{1}\right|+\ldots+|\lambda|\left|a_{s-1}\right|}
$$

Thus,

$$
F_{\sigma, i}(f)\left(a_{1}, \ldots, \lambda a_{s}, \ldots, a_{m}\right)=(-1)^{|\lambda|\left|a_{1}\right|+\ldots+|\lambda|\left|a_{s-1}\right|} \lambda F_{\sigma, i}(f)\left(a_{1}, \ldots, a_{s}, \ldots, a_{m}\right) .
$$

This immediately implies the desired formula (5) and proves the $R^{*}$-linearity of $\Phi_{m}(f)\left(a_{1}, \ldots, a_{m}\right): A^{m} \rightarrow B^{*}$ in each argument. Therefore, we can consider $\Phi_{m}(f)$ as a degree zero $R^{*}$-linear map from $A^{\otimes m}$ to $B^{*}$.

Symmetry. The $R^{*}$-algebra $A^{\otimes m}$ admits a canonical (right) action of the symmetric group $S_{m}$, given by

$$
\tau\left(a_{1} \otimes \ldots \otimes a_{m}\right)=(-1)^{\sum_{p, q=1}^{m} \varepsilon^{p q} \varepsilon_{\tau^{-1}(p) \tau^{-1}(q)}\left|a_{p}\right|\left|a_{q}\right|} a_{\tau(1)} \otimes \ldots \otimes a_{\tau(m)} \quad \forall \tau \in S_{m} .
$$

As usual, we set

$$
\sum_{p, q=1}^{m} \varepsilon^{p q} \varepsilon_{\tau^{-1}(p) \tau^{-1}(q)}\left|a_{p}\right|\left|a_{q}\right|=\varepsilon_{\tau}\left(\left|a_{1}\right|, \ldots,\left|a_{m}\right|\right) .
$$

We need to show that the degree zero $R^{*}$-linear map $\Phi_{m}(f): A^{\otimes m} \rightarrow B^{*}$ is invariant under this action:

$$
\Phi_{m}(f)\left(a_{\tau(1)}, \ldots, a_{\tau(m)}\right)=(-1)^{\varepsilon_{\tau}\left(\left|a_{1}\right|, \ldots,\left|a_{m}\right|\right)} \Phi_{m}(f)\left(a_{1}, \ldots, a_{m}\right) \quad \forall \tau \in S_{m} .
$$

Fix some $\tau \in S_{m}$. Set $b_{s}=a_{\tau(s)}, 1 \leq s \leq m$, which gives $b_{\tau^{-1}(t)}=a_{t}, 1 \leq t \leq m$. Then

$$
\begin{aligned}
\Phi_{m}(f)\left(a_{\tau(1)}, \ldots\right. & \left., a_{\tau(m)}\right)=\Phi_{m}(f)\left(b_{1}, \ldots, b_{m}\right)=\sum_{\sigma \in S_{m}}(-1)^{\sigma}(-1)^{\varepsilon_{i}\left(\left|b_{1}\right|, \ldots,\left|b_{m}\right|\right)} \\
& \times f\left(b_{i_{1}} \ldots b_{i_{k_{1}}}\right) f\left(b_{i_{\left(k_{1}+1\right)}} \ldots b_{i_{\left(k_{1}+k_{2}\right)}}\right) \ldots f\left(b_{i_{\left(k_{1}+\ldots+k_{s-1}+1\right)}} \ldots b_{i_{m}}\right) .
\end{aligned}
$$

Under $\sigma \in S_{m}$, the elements $b_{l}, 1 \leq l \leq m$, are permuted by the rule $\sigma\left(b_{l}\right)=b_{\sigma(l)}$. We want to determine how $\sigma$ acts on the elements $a_{l}, 1 \leq l \leq m$. We have

$$
a_{l}=b_{\tau^{-1}(l)}, \quad \sigma\left(a_{l}\right)=\sigma\left(b_{\tau^{-1}(l)}\right)=b_{\sigma \tau^{-1}(l)}=a_{\tau \sigma \tau^{-1}(l)}, \quad 1 \leq l \leq m .
$$


Thus, $\sigma\left(a_{l}\right)=a_{\tau \sigma \tau^{-1}(l)}, 1 \leq l \leq m$, i.e., each permutation $\sigma \in S_{m}$, viewed as a permutation of $b_{1}, \ldots, b_{m}$, gives rise to the permutation $\sigma^{\prime}=\tau \sigma \tau^{-1}$ of the elements $a_{1}, \ldots, a_{m}$. Notice that $(-1)^{\sigma}=(-1)^{\sigma^{\prime}}$. Therefore, formula (10) can be rewritten as

$$
\begin{aligned}
& \text { (11) } \Phi_{m}(f)\left(b_{1}, \ldots, b_{m}\right)=\sum_{\sigma^{\prime} \in S_{m}}(-1)^{\sigma^{\prime}}\left((-1)^{\varepsilon_{i}\left(\left|b_{1}\right|, \ldots,\left|b_{m}\right|\right)}(-1)^{\varepsilon_{j}\left(\left|a_{1}\right|, \ldots,\left|a_{m}\right|\right)}\right) \\
& \times(-1)^{\varepsilon_{j}\left(\left|a_{1}\right|, \ldots,\left|a_{m}\right|\right)} f\left(a_{j_{1}} \ldots a_{j_{k_{1}}}\right) f\left(a_{j_{\left(k_{1}+1\right)}} \ldots a_{j_{\left(k_{1}+k_{2}\right)}}\right) \ldots f\left(a_{j_{\left(k_{1}+\ldots+k_{s-1}+1\right)}} \ldots a_{j_{m}}\right),
\end{aligned}
$$

where $a_{\tau(s)}=b_{s}, a_{t}=b_{\tau^{-1}(t)}, a_{j_{t}}=b_{i_{t}}, j_{t}=\tau\left(i_{t}\right), 1 \leq t \leq m$, and the permutation $j=(j(1), \ldots, j(m))$ corresponds to $\sigma^{\prime}$. To prove formula (9), we need to show that the resulting sign $(-1)^{\varepsilon_{i}\left(\left|b_{1}\right|, \ldots,\left|b_{m}\right|\right)+\varepsilon_{j}\left(\left|a_{1}\right|, \ldots,\left|a_{m}\right|\right)}$ is exactly $(-1)^{\varepsilon_{\tau}\left(\left|a_{1}\right|, \ldots,\left|a_{m}\right|\right)}$. Let $F A\left(a_{1}^{\prime}, \ldots, a_{m}^{\prime}\right)$ be a free commutative $\mathbb{Q}$-algebra in formal variables $a_{1}^{\prime}, \ldots, a_{m}^{\prime}$, $\left|a_{i}^{\prime}\right|=\left|a_{i}\right|, 1 \leq i \leq m$. Set $b_{s}^{\prime}=a_{\tau(s)}^{\prime}, 1 \leq s \leq m$. By Lemma 1.1.2, we have

$$
\begin{aligned}
b_{i(1)}^{\prime} \ldots b_{i(m)}^{\prime} & =(-1)^{\varepsilon_{i}\left(\left|b_{1}\right|, \ldots,\left|b_{m}\right|\right)} b_{1}^{\prime} \ldots b_{m}^{\prime} \\
& =(-1)^{\varepsilon_{i}\left(\left|b_{1}\right|, \ldots,\left|b_{m}\right|\right)} a_{\tau(1)}^{\prime} \ldots a_{\tau(m)}^{\prime} \\
& =(-1)^{\varepsilon_{i}\left(\left|b_{1}\right|, \ldots,\left|b_{m}\right|\right)+\varepsilon_{\tau}\left(\left|a_{1}\right|, \ldots,\left|a_{m}\right|\right)} a_{1}^{\prime} \ldots a_{m}^{\prime}, \\
b_{i(1)}^{\prime} \ldots b_{i(m)}^{\prime}= & a_{j(1)}^{\prime} \ldots a_{j(m)}^{\prime}=(-1)^{\varepsilon_{j}\left(\left|a_{1}\right|, \ldots,\left|a_{m}\right|\right)} a_{1}^{\prime} \ldots a_{m}^{\prime},
\end{aligned}
$$

which immediately implies the equality of the signs

$$
(-1)^{\varepsilon_{i}\left(\left|b_{1}\right|, \ldots,\left|b_{m}\right|\right)+\varepsilon_{j}\left(\left|a_{1}\right|, \ldots,\left|a_{m}\right|\right)}=(-1)^{\varepsilon_{\tau}\left(\left|a_{1}\right|, \ldots,\left|a_{m}\right|\right)} .
$$

This completes the proof of the proposition.

We want to write down explicit formulas for $\Phi_{1}(f), \Phi_{2}(f)$, and $\Phi_{3}(f)$ :

$$
\begin{gathered}
\Phi_{1}(f)(a)=f(a) \quad \forall a \in A^{*} ; \\
\Phi_{2}(f)\left(a_{1}, a_{2}\right)=f\left(a_{1}\right) f\left(a_{2}\right)-f\left(a_{1} a_{2}\right) \quad \forall a_{1}, a_{2} \in A^{*} ; \\
\Phi_{3}(f)\left(a_{1}, a_{2}, a_{3}\right)=f\left(a_{1}\right) f\left(a_{2}\right) f\left(a_{3}\right)-f\left(a_{1}\right) f\left(a_{2} a_{3}\right)-f\left(a_{1} a_{2}\right) f\left(a_{3}\right) \\
-(-1)^{\left|a_{1}\right|\left|a_{2}\right|} f\left(a_{2}\right) f\left(a_{1} a_{3}\right)+f\left(a_{1} a_{2} a_{3}\right)+(-1)^{\left|a_{1}\right|\left|a_{2}\right|} f\left(a_{2} a_{1} a_{3}\right) \quad \forall a_{1}, a_{2}, a_{3} \in A^{*} .
\end{gathered}
$$

Now we shall prove a key fact.

Proposition 1.1.4. The above maps $\Phi_{m}(f): A^{\otimes m} \rightarrow B^{*}, m \geq 1$, satisfy the following graded Frobenius recursion:

$$
\begin{gathered}
\Phi_{m+1}(f)\left(a_{1}, a_{2}, \ldots, a_{m+1}\right)=f\left(a_{1}\right) \Phi_{m}(f)\left(a_{2}, \ldots, a_{m+1}\right) \\
-\Phi_{m}(f)\left(a_{1} a_{2}, a_{3}, \ldots, a_{m+1}\right)-(-1)^{\left|a_{1}\right|\left|a_{2}\right|} \Phi_{m}(f)\left(a_{2}, a_{1} a_{3}, \ldots, a_{m+1}\right) \\
\quad-(-1)^{\left|a_{1}\right|\left|a_{2}\right|+\left|a_{1}\right|\left|a_{3}\right|} \Phi_{m}(f)\left(a_{2}, a_{3}, a_{1} a_{4}, \ldots, a_{m+1}\right)-\ldots \\
-(-1)^{\left|a_{1}\right|\left|a_{2}\right|+\ldots+\left|a_{1}\right|\left|a_{m}\right|} \Phi_{m}(f)\left(a_{2}, \ldots, a_{m}, a_{1} a_{m+1}\right) \quad \forall a_{1}, \ldots, a_{m} \in A^{*} .
\end{gathered}
$$

Proof. Fix $m \geq 1$. By the definition of $\Phi_{m}(f): A^{\otimes m} \rightarrow B^{*}$, we have

$$
\begin{aligned}
\Phi_{m+1}(f)\left(a_{1}, \ldots, a_{m+1}\right)=\sum_{\sigma \in S_{m+1}} & (-1)^{\sigma}(-1)^{\varepsilon_{i}\left(\left|a_{1}\right|, \ldots,\left|a_{m+1}\right|\right)} \\
& \times f\left(a_{i_{1}} \ldots a_{i_{k_{1}}}\right) \ldots f\left(a_{i_{\left(k_{1}+\ldots+k_{s-1}+1\right)}} \ldots a_{i_{m+1}}\right) .
\end{aligned}
$$

View $S_{m+1}$ as the group Aut $\{1,2, \ldots, m+1\}$, and break the sum $\sum_{\sigma \in S_{m+1}}$ in (1) into $m+1$ summands $\Sigma_{k}=\sum_{\sigma \in S_{m+1}, \sigma(1)=k}(\ldots), 1 \leq k \leq m+1$.

Consider the first sum. We shall identify a permutation $\sigma \in S_{m+1}, \sigma(1)=1$, with $\sigma^{\prime} \in S_{m}=\operatorname{Aut}\{2, \ldots, m+1\}, \sigma^{\prime}(l)=\sigma(l), 2 \leq l \leq m+1$. It is clear that $(-1)^{\sigma}=(-1)^{\sigma^{\prime}}$. 
In formula (1), for each such $\sigma$, we fix a permutation $i=(i(1), \ldots, i(m+1))$ corresponding to $\sigma$ such that $i(1)=1$. Define $i^{\prime} \in S_{m}$ by setting $i^{\prime}(l)=i(l), 2 \leq l \leq m+1$. It is clear that $i^{\prime}=\left(i^{\prime}(2), \ldots, i^{\prime}(m+1)\right)$ corresponds to $\sigma^{\prime}$. Thus, the first sum can be rewritten as

$$
\begin{aligned}
\text { (2) } \quad \Sigma_{1}=\sum_{\sigma \in S_{m+1}, \sigma(1)=1}(-1)^{\sigma} F_{\sigma, i}(f)\left(a_{1}, \ldots, a_{m+1}\right)=\sum_{\sigma^{\prime} \in S_{m}}(-1)^{\sigma^{\prime}}(-1)^{\varepsilon_{i}\left(\left|a_{1}\right|, \ldots,\left|a_{m+1}\right|\right)} \\
\times f\left(a_{1}\right) f\left(a_{i^{\prime}(2)} \ldots a_{i^{\prime}\left(1+k_{2}\right)}\right) \ldots f\left(a_{i^{\prime}\left(k_{1}+\ldots+k_{s-1}+1\right)} \ldots a_{i^{\prime}(m+1)}\right) .
\end{aligned}
$$

Taking $f\left(a_{1}\right)$ out of the sum in $(2)$, we see that if

$$
(-1)^{\varepsilon_{i}\left(\left|a_{1}\right|, \ldots,\left|a_{m+1}\right|\right)}=(-1)^{\varepsilon_{i^{\prime}}\left(\left|a_{2}\right|, \ldots,\left|a_{m+1}\right|\right),}
$$

then $\Sigma_{1}=f\left(a_{1}\right) \Phi_{m}(f)\left(a_{2}, \ldots, a_{m+1}\right)$. As before, let $F A\left(a_{1}^{\prime}, \ldots, a_{m+1}^{\prime}\right),\left|a_{s}^{\prime}\right|=\left|a_{s}\right|$, $1 \leq s \leq m+1$ be a free algebra. By Lemma 1.1.2, we have

$$
\begin{gathered}
a_{1}^{\prime} a_{i^{\prime}(2)}^{\prime} \ldots a_{i^{\prime}(m+1)}^{\prime}=a_{1}^{\prime}(-1)^{\varepsilon_{i^{\prime}}\left(\left|a_{2}\right|, \ldots,\left|a_{m+1}\right|\right)} a_{2}^{\prime} \ldots a_{m+1}^{\prime}, \\
a_{1}^{\prime} a_{i^{\prime}(2)}^{\prime} \ldots a_{i^{\prime}(m+1)}^{\prime}=a_{i(1)}^{\prime} \ldots a_{i(m+1)}^{\prime}=(-1)^{\varepsilon_{i}\left(\left|a_{1}\right|, \ldots,\left|a_{m+1}\right|\right)} a_{1}^{\prime} a_{2}^{\prime} \ldots a_{m+1}^{\prime},
\end{gathered}
$$

which imply the equality $(-1)^{\varepsilon_{i}\left(\left|a_{1}\right|, \ldots,\left|a_{m+1}\right|\right)}=(-1)^{\varepsilon_{i^{\prime}}\left(\left|a_{2}\right|, \ldots,\left|a_{m+1}\right|\right)}$.

Thus, we have proved that $\Sigma_{1}=f\left(a_{1}\right) \Phi_{m}(f)\left(a_{2}, \ldots, a_{m+1}\right)$. Now we fix $k, 2 \leq k \leq$ $m+1$ and, using a similar argument, show that

$$
\Sigma_{k}=-(-1)^{\left|a_{1}\right|\left|a_{2}\right|+\ldots+\left|a_{1}\right|\left|a_{k-1}\right|} \Phi_{m}(f)\left(a_{2}, \ldots, a_{k-1}, a_{1} a_{k}, a_{k+1}, \ldots, a_{m+1}\right) .
$$

We view each permutation $\sigma \in S_{m+1}=\operatorname{Aut}\{1, \ldots, m+1\}$ as a directed graph with vertices $\{1, \ldots, m+1\}$. The vertices $i$ and $j$ are connected by a directed edge $r(i, j)$ if $\sigma(i)=j$. Now collapse the vertices 1 and $k$ into one new vertex with formal label $1 k$, remove the edge $r(1, k)$, and consider the remaining edges as the edges of the new graph. The resulting graph is the graph of some uniquely determined permutation $\sigma^{\prime}$ of the elements $\{2,3, \ldots, k-1,1 k, k+1, \ldots, m+1\}$. It is clear that the obtained correspondence

$$
\mu:\left\{\sigma \in S_{m+1} \mid \sigma(1)=k\right\} \rightarrow S_{m}=\operatorname{Aut}\{2,3, \ldots, m+1\}, \quad \mu(\sigma)=\sigma^{\prime},
$$

is a bijection and that $(-1)^{\sigma}=-(-1)^{\sigma^{\prime}}$. For our permutation $\sigma \in S_{m+1}, \sigma(1)=k$, choose the corresponding permutation $i=(i(1), \ldots, i(m+1))$ such that $i(1)=1$, $i(2)=k$, and set $b_{s}=a_{s}, 2 \leq s \leq m+1, s \neq k, b_{1 k}=a_{1} a_{k}$. We have $m$ elements $b_{2}, \ldots, b_{k-1}, b_{1 k}, b_{k+1}, \ldots, b_{m+1}$ which label the vertices of the new graph. The permutation $\sigma^{\prime} \in S_{m}=\operatorname{Aut}\{2,3, \ldots, k-1,1 k, k+1, \ldots, m+1\}$ acts on these elements. It is clear that, for the permutation $j \in S_{m}$ corresponding to $\sigma^{\prime}$, we can take the one defined by $j(2)=1 k, j(s)=i(s), 3 \leq s \leq m+1$. With that choice, we have

$$
\begin{aligned}
& f\left(a_{i(1)} a_{i(2)} \ldots a_{i\left(k_{1}\right)}\right) f\left(a_{i\left(k_{1}+1\right)} \ldots a_{i\left(k_{1}+k_{2}\right)}\right) \ldots f\left(a_{i\left(k_{1}+\ldots+k_{s-1}+1\right)} \ldots a_{i(m+1)}\right) \\
& =f\left(\left(a_{1} a_{k}\right) \ldots a_{i\left(k_{1}\right)}\right) f\left(a_{i\left(k_{1}+1\right)} \ldots a_{i\left(k_{1}+k_{2}\right)}\right) \ldots f\left(a_{i\left(k_{1}+\ldots+k_{s-1}+1\right)} \ldots a_{i(m+1)}\right) \\
& =f\left(b_{j(2)} \ldots b_{j\left(k_{1}\right)}\right) f\left(b_{j\left(k_{1}+1\right)} \ldots b_{j\left(k_{1}+k_{2}\right)}\right) \ldots f\left(b_{j\left(k_{1}+\ldots+k_{s-1}+1\right)} \ldots b_{j(m+1)}\right) .
\end{aligned}
$$

Thus, our sum $\Sigma_{k}$ can be rewritten as

$$
\begin{aligned}
& \Sigma_{k}=\sum_{\sigma^{\prime} \in S_{m}}-(-1)^{\sigma^{\prime}}\left((-1)^{\varepsilon_{i}\left(\left|a_{1}\right|, \ldots,\left|a_{m+1}\right|\right)}\right. \\
& \left.\times(-1)^{\varepsilon_{j}\left(\left|b_{2}\right|, \ldots,\left|b_{k-1}\right|,\left|b_{1 k}\right|,\left|b_{k+1}\right|, \ldots,\left|b_{m+1}\right|\right)}\right) F_{\sigma^{\prime}, j}(f)\left(b_{2}, \ldots, b_{k-1}, b_{1 k}, b_{k+1}, \ldots, b_{m+1}\right) \\
& =\sum_{\sigma^{\prime} \in S_{m}}-(-1)^{\varepsilon_{i}\left(\left|a_{1}\right|, \ldots,\left|a_{m+1}\right|\right)+\varepsilon_{j}\left(\left|b_{2}\right|, \ldots,\left|b_{m+1}\right|\right)} \\
& \quad \times(-1)^{\sigma^{\prime}} F_{\sigma^{\prime}, j}(f)\left(a_{2}, \ldots, a_{k-1}, a_{1} a_{k}, a_{k+1}, \ldots, a_{m+1}\right) .
\end{aligned}
$$


Therefore, to prove the equality

$$
\Sigma_{k}=-(-1)^{\left|a_{1}\right|\left|a_{2}\right|+\ldots+\left|a_{1}\right|\left|a_{k-1}\right|} \Phi_{m}(f)\left(a_{2}, \ldots, a_{k-1}, a_{1} a_{k}, a_{k+1}, \ldots, a_{m+1}\right),
$$

it suffices to show that

$$
(-1)^{\varepsilon_{i}\left(\left|a_{1}\right|, \ldots,\left|a_{m+1}\right|\right)+\varepsilon_{j}\left(\left|b_{2}\right|, \ldots,\left|b_{k-1}\right|,\left|b_{1 k}\right|,\left|b_{k+1}\right|, \ldots,\left|b_{m+1}\right|\right)}=(-1)^{\left|a_{1}\right|\left|a_{2}\right|+\ldots+\left|a_{1}\right|\left|a_{k-1}\right|} .
$$

As usual, let $F A\left(a_{1}^{\prime}, \ldots, a_{m+1}^{\prime}\right),\left|a_{s}^{\prime}\right|=\left|a_{s}\right|, 1 \leq s \leq m+1$ be a free algebra and set $b_{1 k}^{\prime}=a_{1}^{\prime} a_{k}^{\prime}, b_{s}^{\prime}=a_{s}^{\prime}, 2 \leq s \leq m+1, s \neq k$. By Lemma 1.1.2, we have

$$
\begin{gathered}
a_{i(1)}^{\prime} \ldots a_{i(m+1)}^{\prime}=(-1)^{\varepsilon_{i}\left(\left|a_{1}\right|, \ldots,\left|a_{m+1}\right|\right)} a_{1} a_{2} \ldots a_{m+1} \\
a_{i(1)}^{\prime} \ldots a_{i(m+1)}^{\prime} \\
=b_{j(2)}^{\prime} b_{j(3)}^{\prime} \ldots b_{j(m+1)}^{\prime} \\
=(-1)^{\varepsilon_{j}\left(\left|b_{2}\right|, \ldots,\left|b_{k-1}\right|,\left|b_{1 k}\right|,\left|b_{k+1}\right|, \ldots,\left|b_{m+1}\right|\right)} b_{2}^{\prime} \ldots b_{k-1}^{\prime} b_{1 k}^{\prime} b_{k+1}^{\prime} \ldots b_{m+1}^{\prime} .
\end{gathered}
$$

Moreover,

$$
\begin{aligned}
b_{2}^{\prime} b_{3}^{\prime} \ldots b_{k-1}^{\prime} b_{1 k}^{\prime} b_{k+1}^{\prime} \ldots b_{m+1}^{\prime}=a_{2} \ldots a_{k-1} a_{1} a_{k} a_{k+1} \ldots a_{m+1} \\
\quad=(-1)^{\left|a_{1}\right|\left|a_{2}\right|+\ldots+\left|a_{1}\right|\left|a_{k-1}\right|} a_{1} a_{2} \ldots a_{m+1} .
\end{aligned}
$$

Now formulas (4) and (5) immediately imply the equality

$$
(-1)^{\varepsilon_{i}\left(\left|a_{1}\right|, \ldots,\left|a_{m+1}\right|\right)+\varepsilon_{j}\left(\left|b_{2}\right|, \ldots,\left|b_{k-1}\right|,\left|b_{1 k}\right|,\left|b_{k+1}\right|, \ldots,\left|b_{m+1}\right|\right)}=(-1)^{\left|a_{1}\right|\left|a_{2}\right|+\ldots+\left|a_{1}\right|\left|a_{k-1}\right|},
$$

which, in turn, proves the equality

$$
\Sigma_{k}=-(-1)^{\left|a_{1}\right|\left|a_{2}\right|+\ldots+\left|a_{1}\right|\left|a_{k-1}\right|} \Phi_{m}(f)\left(a_{2}, \ldots, a_{k-1}, a_{1} a_{k}, a_{k+1}, \ldots, a_{m+1}\right)
$$

for any $k, 2 \leq k \leq m+1$. Since $\Phi_{m+1}(f)\left(a_{1}, \ldots, a_{m+1}\right)=\Sigma_{1}+\sum_{k=2}^{m+1} \Sigma_{k}$, Proposition 1.1.4 is completely proved.

\subsection{Graded Frobenius $n$-homomorphisms.}

Definition 1.2.1. Suppose $A^{*}$ and $B^{*}$ are $R^{*}$-algebras, $B^{*}$ is commutative, and $f: A^{*} \rightarrow$ $B^{*}$ is a degree zero $R^{*}$-linear map. Then $f$ is called a Frobenius $n$-homomorphism if:

(1) $f(a b)=(-1)^{|a||b|} f(b a) \quad \forall a, b \in A^{*}$;

(2) $f(1)=n$;

(3) $\Phi_{n+1}(f)\left(a_{1}, a_{2}, \ldots, a_{n+1}\right)=0 \quad \forall a_{1}, \ldots, a_{n+1} \in A^{*}$.

Remark 1. It follows easily from Proposition 1.1.4 that for any $n$-homomorphism $f: A^{*} \rightarrow$ $B^{*}$ we have $\Phi_{n+k}(f)\left(a_{1}, a_{2}, \ldots, a_{n+k}\right)=0 \forall k \geq 1, \forall a_{1}, \ldots, a_{n+k} \in A^{*}$.

Remark 2. The concepts of 1-homomorphism and algebra homomorphism coincide.

Suppose $A^{*}$ and $B^{*}$ are $R^{*}$-algebras, $B^{*}$ is commutative, $f, g: A^{*} \rightarrow B^{*}$ are degree zero $R^{*}$-linear maps such that $f(a b)=(-1)^{|a||b|} f(b a) \forall a, b \in A^{*}$, and $g(a b)=(-1)^{|a||b|} g(b a)$ $\forall a, b \in A^{*}$.

Fix an arbitrary natural number $m$ and let $E$ denote the set $\{1,2, \ldots, m\}$. For any homogeneous elements $a_{1}, \ldots, a_{m} \in A^{*}$ and any $I \subset E, I=\left\{i_{1}, \ldots, i_{k}\right\}, 1 \leq i_{1}<$ $i_{2}<\ldots<i_{k} \leq m$, set $\Phi_{|I|}(f)\left(a_{i} \mid i \in I\right):=\Phi_{|I|}(f)\left(a_{i_{1}}, a_{i_{2}}, \ldots, a_{i_{k}}\right)$. If $I=\varnothing$, we set $\Phi_{|\varnothing|}(f)\left(a_{i} \mid i \in \varnothing\right)=1$.

Lemma 1.2.2. Under the above assumptions, $\forall I \subset E, \forall k_{1}, \ldots, k_{m} \in \mathbb{Z}_{+}$there are numbers $\nu_{I ; k_{1}, \ldots, k_{m}} \in\{0,1\}$, such that

$$
\begin{gathered}
\Phi_{m}(f+g)\left(a_{1}, \ldots, a_{m}\right)=\sum_{I \subset E}(-1)^{\nu_{I ;\left|a_{1}\right|, \ldots,\left|a_{m}\right|} \mid} \Phi_{|I|}(f)\left(a_{i} \mid i \in I\right) \Phi_{|E \backslash I|}(g)\left(a_{i} \mid i \in E \backslash I\right) \\
\forall a_{1}, \ldots, a_{m} \in A^{*}
\end{gathered}
$$


Proof. By the definition of $\Phi_{m}(f)$,

$$
\begin{aligned}
\Phi_{m}(f+g)\left(a_{1}, \ldots, a_{m}\right) & \\
= & \sum_{\sigma \in S_{m}}(-1)^{\sigma}(-1)^{\varepsilon_{i}\left(\left|a_{1}\right|, \ldots,\left|a_{m}\right|\right)}\left(f\left(a_{i(1)} \ldots a_{i\left(k_{1}\right)}\right)+g\left(a_{i(1)} \ldots a_{i\left(k_{1}\right)}\right)\right) \\
& \quad \times\left(f\left(a_{i\left(k_{1}+1\right)} \ldots a_{i\left(k_{1}+k_{2}\right)}\right)+g\left(a_{i\left(k_{1}+1\right)} \ldots a_{i\left(k_{1}+k_{2}\right)}\right)\right) \times \ldots \\
\quad & \times\left(f\left(a_{i\left(k_{1}+\ldots+k_{s-1}+1\right)} \ldots a_{i(m)}\right)+g\left(a_{i\left(k_{1}+\ldots+k_{s-1}+1\right)} \ldots a_{i(m)}\right)\right) .
\end{aligned}
$$

Fix some $\sigma \in S_{m}$ and look at the product of the $s$ parenthesized expressions, together with the preceding $\operatorname{sign}(-1)^{\varepsilon_{i}\left(\left|a_{1}\right|, \ldots,\left|a_{m}\right|\right)}$. Our permutation $\sigma$ is the product of $s$ disjoint cycles $\gamma_{1}, \ldots, \gamma_{s}$. A choice of the permutation $i=(i(1), \ldots, i(m))$ is equivalent to a choice of order on the set of cycles $\left\{\gamma_{1}, \ldots, \gamma_{s}\right\}$ and a choice of an initial element $i_{k_{1}+\ldots+k_{t-1}+1}$ in each cycle $\gamma_{t}, 1 \leq t \leq s$. Each parenthesized expression in our product is labeled by some cycle $\gamma_{t}, 1 \leq t \leq s$, and the order of those expressions corresponds to the order of the cycles. If we distribute all $s$ factors, we will have the following sum of $2^{s}$ terms:

$$
\begin{aligned}
\sum_{\left(h_{1}, \ldots, h_{s}\right) \in\{f, g\}^{s}}(-1)^{\varepsilon_{i}\left(\left|a_{1}\right|, \ldots,\left|a_{m}\right|\right)} h_{1}\left(a_{i(1)} \ldots a_{i\left(k_{1}\right)}\right) & h_{2}\left(a_{i\left(k_{1}+1\right)} \ldots a_{i\left(k_{1}+k_{2}\right)}\right) \times \ldots \\
& \times h_{s}\left(a_{i\left(k_{1}+\ldots+k_{s-1}+1\right)} \ldots a_{i(m)}\right),
\end{aligned}
$$

where the vector of symbols $\left(h_{1}, \ldots, h_{s}\right)$ runs through $\{f, g\}^{s}$. In fact, in each of these $2^{s}$ terms, for each cycle $\gamma_{t}$ we choose either $h_{t}=f$ or $g$. Assume such a choice is made for each cycle $\gamma_{t}, 1 \leq t \leq s$, and examine our product

$$
\begin{aligned}
(-1)^{\varepsilon_{i}\left(\left|a_{1}\right|, \ldots,\left|a_{m}\right|\right)} h_{1}\left(a_{i(1)} \ldots a_{i\left(k_{1}\right)}\right) h_{2}\left(a_{i\left(k_{1}+1\right)}\right. & \left.\ldots a_{i\left(k_{1}+k_{2}\right)}\right) \times \ldots \\
& \times h_{s}\left(a_{i\left(k_{1}+\ldots+k_{s-1}+1\right)} \ldots a_{i(m)}\right) .
\end{aligned}
$$

Now order our cycles $\gamma_{1}, \ldots, \gamma_{s}$ in some other way, i.e., set $\gamma_{u}=\gamma_{j^{-1}(u)}^{\prime}, 1 \leq u \leq s$, where $j \in \operatorname{Aut}\{1, \ldots, s\}$. In each cycle $\gamma_{t}^{\prime}=\gamma_{j(t)}$, choose a (possibly) different initial element. This is equivalent to a choice of another permutation $i^{\prime}=\left(i^{\prime}(1), \ldots, i^{\prime}(m)\right) \in$ $S_{m}$, corresponding to $\sigma$. Then, in the new sum of the $2^{s}$ terms corresponding to $i^{\prime}$, the summand corresponding to (1) will be

$$
\begin{aligned}
(-1)^{\varepsilon_{i^{\prime}}\left(\left|a_{1}\right|, \ldots,\left|a_{m}\right|\right)} h_{j(1)}\left(a_{i^{\prime}(1)} \ldots a_{i^{\prime}\left(k_{j(1)}\right)}\right) h_{j(2)} & \left(a_{i^{\prime}\left(k_{j(1)}+1\right)} \ldots a_{i^{\prime}\left(k_{j(1)}+k_{j(2)}\right)}\right) \times \ldots \\
& \times h_{j(s)}\left(a_{i^{\prime}\left(k_{j(1)}+\ldots+k_{j(s-1)}+1\right)} \ldots a_{i^{\prime}(m)}\right) .
\end{aligned}
$$

It is not difficult to check that these two summands are equal. Indeed, it is clear that for any new permutation $i^{\prime}$, such summands differ by a factor $(-1)^{\varepsilon_{\left(i, i^{\prime}\right)}\left(\left|a_{1}\right|, \ldots,\left|a_{m}\right|\right)}$. To see that, notice that a permutation of two adjacent cycles $\gamma_{t}$ and $\gamma_{t+1}$ removes a certain $\operatorname{sign}\left(B^{*}\right.$ is a commutative algebra) and a choice of the initial element in each cycle $\gamma_{t}$ also removes a sign because $h_{t}(a b)=(-1)^{|a||b|} h_{t}(b a), 1 \leq t \leq s$. We also see that the sign $(-1)^{\varepsilon_{\left(i, i^{\prime}\right)}\left(\left|a_{1}\right|, \ldots,\left|a_{m}\right|\right)}$ equals (by construction) the ratio of the following elements:

$$
\begin{gathered}
(-1)^{\varepsilon_{i}\left(\left|a_{1}\right|, \ldots,\left|a_{m}\right|\right)} a_{i(1)}^{\prime} \ldots a_{i(m)}^{\prime}, \\
(-1)^{\varepsilon_{i^{\prime}}\left(\left|a_{1}\right|, \ldots,\left|a_{m}\right|\right)} a_{i^{\prime}(1)}^{\prime} \ldots a_{i^{\prime}(m)}^{\prime}
\end{gathered}
$$

in the free algebra $F A\left(a_{1}^{\prime}, \ldots, a_{m}^{\prime}\right),\left|a_{i}^{\prime}\right|=\left|a_{i}\right|, 1 \leq i \leq m$. By Lemma 1.1.2, these elements coincide with $a_{1}^{\prime} \ldots a_{m}^{\prime}$; i.e., the $\operatorname{sign}(-1)^{\varepsilon_{\left(i, i^{\prime}\right)}\left(\left|a_{1}\right|, \ldots,\left|a_{m}\right|\right)}$ is always +1 . Thus, we have proved that for any two permutations $i=(i(1), \ldots, i(m))$ and $i^{\prime}=\left(i^{\prime}(1), \ldots, i^{\prime}(m)\right)$ corresponding to $\sigma$, the summands corresponding to each other in the two sums are equal. Therefore, for each of the $2^{s}$ terms, we can assign its own permutation $i=$ $(i(1), \ldots, i(m))$. 
More precisely, for a given term $\left(h_{1}, \ldots, h_{s}\right) \in\{f, g\}^{s}$, choose an ordering of the cycles $\gamma_{1}^{\prime}=\gamma_{j(1)}, \ldots, \gamma_{s}^{\prime}=\gamma_{j(s)}$ in such a way that the vector $\left(h_{j(1)}, \ldots, h_{j(s)}\right)$ is of the form $(f, \ldots, f, g, \ldots, g)$, i.e., first all the $f$, then all the $g$. Thus, we have the following formula (here $E=\{1, \ldots, m\}$ ):

$$
\begin{gathered}
\Phi_{m}(f+g)\left(a_{1}, \ldots, a_{m}\right)=\sum_{\sigma \in S_{m}} \sum_{I \subset E, \sigma(I)=I}(-1)^{\sigma}(-1)^{\varepsilon_{i}\left(\left|a_{1}\right|, \ldots,\left|a_{m}\right|\right)} f\left(a_{i(1)} \ldots a_{i\left(k_{1}\right)}\right) \times \ldots \\
\times f\left(a_{i\left(k_{1}+\ldots+k_{r-1}+1\right)} \ldots a_{i\left(k_{1}+\ldots+k_{r}\right)}\right) g\left(a_{i\left(k_{1}+\ldots+k_{r}+1\right)} \ldots a_{i\left(k_{1}+\ldots+k_{r}+k_{r+1}\right)}\right) \times \ldots \\
\times g\left(a_{i\left(k_{1}+\ldots+k_{s-1}+1\right)} \ldots a_{i(m)}\right)
\end{gathered}
$$

where the inner sum of the $2^{s}$ elements is written in the form $\sum_{I \subset E, \sigma(I)=I}(\ldots)$ because a choice of the cycles $\gamma_{t}$ for which $h_{t}=f$ is equivalent to a choice of the union of those cycles as a $\sigma$-invariant subset $I \subset E$ of indices. Consider an arbitrary $I \subset E$ and write in the increasing order the indices from $I$ and $E \backslash I$ :

$$
\begin{gathered}
\left\{i_{0}^{\prime}(1), i_{0}^{\prime}(2), \ldots, i_{0}^{\prime}(r)\right\}=I, \quad 1 \leq i_{0}^{\prime}(1)<\ldots<i_{0}^{\prime}(r) \leq m, \quad|I|=r, \\
\left\{i_{0}^{\prime \prime}(1), i_{0}^{\prime \prime}(2), \ldots, i_{0}^{\prime \prime}(m-r)\right\}=E \backslash I, \quad 1 \leq i_{0}^{\prime \prime}(1)<\ldots<i_{0}^{\prime \prime}(m-r) \leq m .
\end{gathered}
$$

This will be the initial ordering of these index sets. It is clear that a choice of permutation $\sigma \in S_{m}$ such that $\sigma(I)=I$ is equivalent to a choice of two permutations $\sigma_{1} \in \operatorname{Aut}(I)$ and $\sigma_{2} \in \operatorname{Aut}(E \backslash I)$. Moreover, $(-1)^{\sigma}=(-1)^{\sigma_{1}}(-1)^{\sigma_{2}}$. Now fix a pair of permutations $\sigma_{1} \in \operatorname{Aut}(I)$ and $\sigma_{2} \in \operatorname{Aut}(E \backslash I)$ and consider the corresponding permutations $i^{\prime}=\left(i^{\prime}(1), \ldots, i^{\prime}(r)\right) \in \operatorname{Aut}(I)$ and $i^{\prime \prime}=\left(i^{\prime \prime}(1), \ldots, i^{\prime \prime}(m-r)\right) \in \operatorname{Aut}(E \backslash I)$. A general permutation $i=(i(1), \ldots, i(m))$ corresponding to $\sigma=\left(\sigma_{1}, \sigma_{2}\right)$ is of the form $i=\left(i^{\prime}, i^{\prime \prime}\right)=\left(i^{\prime}(1), \ldots, i^{\prime}(r), i^{\prime \prime}(1), \ldots, i^{\prime \prime}(m-r)\right)$. Thus, if in formula (2) we interchange two signs in the sum, we have

$$
\begin{aligned}
& \Phi_{m}(f+g)\left(a_{1}, \ldots, a_{m}\right)=\sum_{I \subset E} \sum_{\sigma_{1} \in \operatorname{Aut}(I), \sigma_{2} \in \operatorname{Aut}(E \backslash I)}(-1)^{\sigma_{1}}(-1)^{\sigma_{2}} \\
& \times\left((-1)^{\varepsilon_{i}\left(\left|a_{1}\right|, \ldots,\left|a_{m}\right|\right)}(-1)^{\varepsilon_{i^{\prime}}\left(\left|a_{i_{0}^{\prime}(1)}\right|, \ldots,\left|a_{i_{0}^{\prime}(r)}\right|\right)}(-1)^{\varepsilon_{i^{\prime \prime}}\left(\left|a_{i_{0}^{\prime \prime}(1)}\right|, \ldots,\left|a_{i_{0}^{\prime \prime}(m-r)}\right|\right)}\right) \\
& \times(-1)^{\varepsilon_{i^{\prime}}\left(\left|a_{i_{0}^{\prime}(1)}\right|, \ldots,\left|a_{i_{0}^{\prime}(r)}\right|\right)}(-1)^{\varepsilon_{i^{\prime \prime}}\left(\left|a_{i_{0}^{\prime \prime}(1)}\right|, \ldots,\left|a_{i_{0}^{\prime \prime}(m-r)}\right|\right)} \\
& \times f\left(a_{i^{\prime}(1)} \ldots a_{i^{\prime}\left(k_{1}\right)}\right) \ldots f\left(a_{i^{\prime}\left(k_{1}+\ldots+k_{\left(s^{\prime}-1\right)}+1\right)} \ldots a_{i^{\prime}(r)}\right)
\end{aligned}
$$

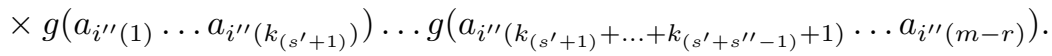

If we now prove that the sign

$$
(-1)^{\varepsilon_{i}\left(\left|a_{1}\right|, \ldots,\left|a_{m}\right|\right)}(-1)^{\varepsilon_{i^{\prime}}\left(\left|a_{i_{0}^{\prime}(1)}\right|, \ldots,\left|a_{i_{0}^{\prime}(r)}\right|\right)}(-1)^{\varepsilon_{i^{\prime \prime}}\left(\left|a_{i_{0}^{\prime \prime}(1)}\right|, \ldots,\left|a_{i_{0}^{\prime \prime}(m-r)}\right|\right)}
$$

equals $(-1)^{\nu_{I} ;\left|a_{1}\right|, \ldots,\left|a_{m}\right|}$ for some number $\nu_{I ;\left|a_{1}\right|, \ldots,\left|a_{m}\right|} \in\{0,1\}$, depending on $I \subset E$ and the degrees $\left|a_{1}\right|, \ldots,\left|a_{m}\right|$, then, moving it past the sign of the inner sum in (3), we would have the assertion of the lemma:

$$
\begin{aligned}
\Phi_{m}(f+g)\left(a_{1}, \ldots, a_{m}\right)=\sum_{I \subset E}(-1)^{\nu_{I ;\left|a_{1}\right|, \ldots,\left|a_{m}\right|}} \Phi_{|I|}(f)\left(a_{i_{0}^{\prime}(1)}, \ldots, a_{i_{0}^{\prime}(r)}\right) \\
\\
\times \Phi_{|E \backslash I|}(g)\left(a_{i_{0}^{\prime \prime}(1)}, \ldots, a_{i_{0}^{\prime \prime}(m-r)}\right) .
\end{aligned}
$$

We want to prove now that sign (4) depends only on $I \subset E$ and the degrees $\left|a_{1}\right|, \ldots$, $\left|a_{m}\right|$. Consider a free commutative graded algebra $F A\left(a_{1}^{\prime}, \ldots, a_{m}^{\prime}\right)$ over $\mathbb{Q}$ in formal 
variables $a_{1}^{\prime}, \ldots, a_{m}^{\prime},\left|a_{i}^{\prime}\right|=\left|a_{i}\right|, 1 \leq i \leq m$. By Lemma 1.1.2,

$$
\begin{aligned}
& a_{i(1)}^{\prime} \ldots a_{i(m)}^{\prime}=(-1)^{\varepsilon_{i}\left(\left|a_{1}\right|, \ldots,\left|a_{m}\right|\right)} a_{1}^{\prime} \ldots a_{m}^{\prime}, \\
& a_{i(1)}^{\prime} \ldots a_{i(m)}^{\prime}=a_{i^{\prime}(1)}^{\prime} \ldots a_{i^{\prime}(r)}^{\prime} a_{i^{\prime \prime}(1)}^{\prime} \ldots a_{i^{\prime \prime}(m-r)}^{\prime} \\
& =(-1)^{\varepsilon_{i^{\prime}}\left(\left|a_{i_{0}^{\prime}(1)}\right|, \ldots,\left|a_{i_{0}^{\prime}(r)}\right|\right)}(-1)^{\varepsilon_{i^{\prime \prime}}\left(\left|a_{i_{0}^{\prime \prime}(1)}\right|, \ldots,\left|a_{i_{0}^{\prime \prime}(m-r)}\right|\right)} a_{i_{0}^{\prime}(1)}^{\prime} \ldots a_{i_{0}^{\prime}(r)}^{\prime} a_{i_{0}^{\prime \prime}(1)}^{\prime} \ldots a_{i_{0}^{\prime \prime}(m-r)}^{\prime}, \\
& \\
& a_{i_{0}^{\prime}(1)}^{\prime} \ldots a_{i_{0}^{\prime}(r)}^{\prime} a_{i_{0}^{\prime \prime}(1)}^{\prime} \ldots a_{i_{0}^{\prime \prime}(m-r)}^{\prime}=(-1)^{\varepsilon_{i_{0}=\left(i_{0}^{\prime}, i_{0}^{\prime \prime}\right)}\left(\left|a_{1}\right|, \ldots,\left|a_{m}\right|\right)} a_{1}^{\prime} \ldots a_{m}^{\prime} .
\end{aligned}
$$

Set $(-1)^{\varepsilon_{i_{0}=\left(i_{0}^{\prime}, i_{0}^{\prime \prime}\right)}\left(\left|a_{1}\right|, \ldots,\left|a_{m}\right|\right)}=(-1)^{\nu_{I ;}\left|a_{1}\right|, \ldots,\left|a_{m}\right|}$. It now follows at once from the above formulas that (4) equals $(-1)^{\nu_{I} ;\left|a_{1}\right|, \ldots,\left|a_{m}\right|}$. This proves the lemma.

Theorem 1.2.3. Suppose $A^{*}$ and $B^{*}$ are $R^{*}$-algebras, $B^{*}$ is commutative, $f: A^{*} \rightarrow B^{*}$ is an $n$-homomorphism, and $g: A^{*} \rightarrow B^{*}$ is an m-homomorphism. Then $f+g: A^{*} \rightarrow B^{*}$ is an $(n+m)$-homomorphism.

Proof. We need to verify conditions (1), (2) and (3) of Definition 1.2.1 for $f+g: A^{*} \rightarrow B^{*}$. Conditions (1) and (2) obviously hold. Now let us verify condition (3). Indeed, by the previous lemma,

$$
\begin{gathered}
\text { (*) } \Phi_{n+m+1}(f+g)\left(a_{1}, \ldots, a_{n+m+1}\right) \\
=\sum_{I \subset E=\{1, \ldots, n+m+1\}}(-1)^{\nu_{I ;\left|a_{1}\right|, \ldots,\left|a_{n+m+1}\right|} \Phi_{|I|}(f)\left(a_{i} \mid i \in I\right) \Phi_{|E \backslash I|}(g)\left(a_{i} \mid i \in E \backslash I\right)} \\
\forall a_{1}, \ldots, a_{n+m+1} \in A^{*} .
\end{gathered}
$$

We can have two possibilities for $I \subset E$ : 1) $|I| \geq n+1$, in which case the factor $\Phi_{|I|}(f)\left(a_{i} \mid i \in I\right)$ vanishes, and 2) $|I| \leq n,|E \backslash I| \geq m+1$, in which case the factor $\Phi_{|E \backslash I|}(g)\left(a_{i} \mid i \in E \backslash I\right)$ vanishes. Thus, for any $I \subset E$, the corresponding summand in (*) is zero and therefore, $\Phi_{n+m+1}(f+g)\left(a_{1}, \ldots, a_{n+m+1}\right)=0 \forall a_{1}, \ldots, a_{n+m+1} \in A^{*}$, as required.

Remark. In the nongraded case, this theorem was proved by V. M. Buchstaber and E. G. Rees in 10.

Theorem 1.2 .3 allows us to construct new $n$-homomorphisms from old ones. In particular, the map $f=f_{1}+\ldots+f_{n}: A^{*} \rightarrow B^{*}$, where $f_{i}: A^{*} \rightarrow B^{*}, 1 \leq i \leq n$, are algebra homomorphisms, is an $n$-homomorphism. The next result also allows us to construct new $n$-homomorphisms. Suppose $A^{*}$ and $A^{\prime *}$ are (possibly, noncommutative) $R^{*}$-algebras and $B^{*}$ and $B^{\prime *}$ are commutative $R^{*}$-algebras.

Proposition 1.2.4. (i) Suppose $j: A^{*} \rightarrow A^{*}$ is an algebra homomorphism and $f: A^{*} \rightarrow$ $B^{*}$ is an n-homomorphism. Then $f \circ j: A^{* *} \rightarrow B^{*}$ is an n-homomorphism.

(ii) Suppose $f: A^{*} \rightarrow B^{*}$ is an n-homomorphism and $j: B^{*} \rightarrow B^{*}$ is an algebra homomorphism. Then $j \circ f: A^{*} \rightarrow B^{\prime *}$ is an n-homomorphism. Moreover, if $j: B^{*} \rightarrow B^{\prime *}$ is a monomorphism and, for a degree zero $R^{*}$-linear map $f: A^{*} \rightarrow B^{*}$, the composition $j \circ f: A^{*} \rightarrow B^{\prime *}$ is an n-homomorphism, then $f: A^{*} \rightarrow B^{*}$ itself is an n-homomorphism.

Proof. (i) It is clear that $f \circ j: A^{\prime *} \rightarrow B^{*}$ is a degree zero $R^{*}$-linear map. We want to check that it satisfies the conditions of Definition 1.2.1. For (1) and (2), we have:

(1) $(f \circ j)(a b)=f(j(a b))=f(j(a) j(b))=f\left((-1)^{|a||b|} j(b) j(a)\right)=(-1)^{|a||b|} \times f(j(b a))=$ $(-1)^{|a||b|}(f \circ j)(b a) \forall a, b \in A^{\prime *}$

(2) $(f \circ j)(1)=f(j(1))=f(1)=n$. 
Now we check (3):

$$
\begin{aligned}
& \Phi_{n+1}(f \circ j)\left(a_{1}, \ldots, a_{n+1}\right) \\
& =\sum_{\sigma \in S_{n+1}}(-1)^{\sigma}(-1)^{\varepsilon_{i}\left(\left|a_{1}\right|, \ldots,\left|a_{n+1}\right|\right)} f\left(j\left(a_{i_{1}} \ldots a_{i_{k_{1}}}\right)\right) \ldots f\left(j\left(a_{i_{\left(k_{1}+\ldots+k_{s-1}+1\right)}} \ldots a_{i_{n+1}}\right)\right) \\
& =\sum_{\sigma \in S_{n+1}}(-1)^{\sigma}(-1)^{\varepsilon_{i}\left(\left|j\left(a_{1}\right)\right|, \ldots,\left|j\left(a_{n+1}\right)\right|\right)} f\left(j\left(a_{i_{1}}\right) \ldots j\left(a_{i_{k_{1}}}\right)\right) \times \ldots \\
& \quad \times f\left(j \left(a_{\left.\left.i_{\left(k_{1}+\ldots+k_{s-1}+1\right)}\right) \ldots j\left(a_{i_{n+1}}\right)\right)}\right.\right. \\
& =\Phi_{n+1}(f)\left(j\left(a_{1}\right), \ldots, j\left(a_{n+1}\right)\right)=0 \quad \forall a_{1}, \ldots, a_{n+1} \in A^{\prime *} .
\end{aligned}
$$

(ii) We begin with the first assertion. It is clear that $j \circ f: A^{*} \rightarrow B^{\prime *}$ is a degree zero $R^{*}$-linear map. We want to check that it satisfies the conditions of Definition 1.2.1. For (1) and (2) we have:

(1) $(j \circ f)(a b)=j(f(a b))=j\left((-1)^{|a||b|} f(b a)\right)=(-1)^{|a||b|}(j \circ f)(b a) \forall a, b \in A^{*}$;

(2) $(j \circ f)(1)=j(f(1))=j(n)=n j(1)=n$.

Now we check $(3)$ :

$$
\begin{array}{r}
\Phi_{n+1}(j \circ f)\left(a_{1}, \ldots, a_{n+1}\right)=\sum_{\sigma \in S_{n+1}}(-1)^{\sigma}(-1)^{\varepsilon_{i}\left(\left|a_{1}\right|, \ldots,\left|a_{n+1}\right|\right)} j\left(f\left(a_{i_{1}} \ldots a_{i_{k_{1}}}\right)\right) \times \ldots \\
\times j\left(f\left(a_{i_{\left(k_{1}+\ldots+k_{s-1}+1\right)}} \ldots a_{i_{n+1}}\right)\right) \\
=j\left(\Phi_{n+1}(f)\left(a_{1}, \ldots, a_{n+1}\right)\right)=j(0)=0 \quad \forall a_{1}, \ldots, a_{n+1} \in A^{*} .
\end{array}
$$

Thus, we have proved the first assertion of (ii). Suppose now that $j: B^{*} \rightarrow B^{\prime *}$ is a monomorphism, and that, for a degree zero $R^{*}$-linear map $f: A^{*} \rightarrow B^{*}$, the composition $j \circ f: A^{*} \rightarrow B^{\prime *}$ is an $n$-homomorphism. By the definition of an $n$-homomorphism and since $j$ is a monomorphism, we have

$$
\begin{gathered}
(j \circ f)(a b)=(-1)^{|a||b|}(j \circ f)(b a), \quad j(f(a b))=j\left((-1)^{|a||b|} f(b a)\right), \\
f(a b)=(-1)^{|a||b|} f(b a) \quad \forall a, b \in A^{*} ; \\
(j \circ f)(1)=n, \quad j(f(1))=j(n)=n, \quad f(1)=n ; \\
\Phi_{n+1}(j \circ f)\left(a_{1}, \ldots, a_{n+1}\right)=j\left(\Phi_{n+1}(f)\left(a_{1}, \ldots, a_{n+1}\right)\right)=0 \quad \forall a_{1}, \ldots, a_{n+1} \in A^{*}, \\
\Phi_{n+1}(f)\left(a_{1}, \ldots, a_{n+1}\right)=0 \quad \forall a_{1}, \ldots, a_{n+1} \in A^{*} .
\end{gathered}
$$

This finishes the proof of the proposition.

\section{Cohomology of BRAnChed COVERINGS}

\subsection{Definition and basic properties of $n$-transfer.}

Definition 2.1.1. Suppose $B^{*}$ and $A^{*}$ are commutative graded rings with identities, $1_{A} \neq 0,1_{B} \neq 0,1 / n ! \in A^{0}, B^{0}$, and $i: B^{*} \rightarrow A^{*}$ is a ring homomorphism, making $A^{*}$ into a $B^{*}$-algebra. A degree zero $B^{*}$-linear map $\tau: A^{*} \rightarrow B^{*}$ is called an $n$-transfer (with respect to $\left.i: B^{*} \rightarrow A^{*}\right)$, if:

(1) $\tau: A^{*} \rightarrow B^{*}$ is an $n$-homomorphism of $B^{*}$-algebras;

(2) $i \circ \tau=\operatorname{Id}_{A^{*}}+g$, where $g: A^{*} \rightarrow A^{*}$ is a uniquely defined $(n-1)$-homomorphism.

Remark. In the nongraded case, the notion of $n$-transfer was introduced by V. M. Buchstaber and E. G. Rees in [8].

We begin by describing the additive information carried by an $n$-transfer.

Proposition 2.1.2. Suppose $i: B^{*} \rightarrow A^{*}, \tau: A^{*} \rightarrow B^{*}$ is an $n$-transfer. Then the ring homomorphism $i$ is an embedding and the $B^{*}$-linear map $\tau / n: A^{*} \rightarrow B^{*} \subset A^{*}$ is a 
projector to the submodule $B^{*} \subset A^{*}$. Suppose $L^{*}=\operatorname{Ker} \tau \subset A^{*}$. Then we have a direct sum decomposition $A^{*}=B^{*} \oplus L^{*}$ of $B^{*}$-submodules.

Proof. Consider the composition $\tau \circ i: B^{*} \rightarrow B^{*}$. Since $i: B^{*} \rightarrow A^{*}$ is a $B^{*}$-linear 1-homomorphism and $\tau: A^{*} \rightarrow B^{*}$ is a $B^{*}$-linear $n$-homomorphism, the composition $\tau \circ i: B^{*} \rightarrow B^{*}$ is an $n$-homomorphism of $B^{*}$-algebras. Therefore, $\tau \circ i(b)=\tau \circ i(b \cdot 1)=$ $b \cdot(\tau \circ i(1))=n b \forall b \in B^{*}$. Since $1 / n ! \in B^{0}$, the ring homomorphism $i: B^{*} \rightarrow A^{*}$ is an embedding, and thus we can identify $B^{*}$ with a subring of $A^{*}$.

Then $(\tau / n)(b)=b \forall b \in B^{*} \subset A^{*}$. It now follows that $\tau / n: A^{*} \rightarrow B^{*} \subset A^{*}$ is a $B^{*}$-linear projector to the $B^{*}$-submodule $B^{*}$. Thus, we have a direct sum decomposition $A^{*}=B^{*} \oplus L^{*}$ of $B^{*}$-submodules, where $L^{*}=\operatorname{Ker}(\tau / n)=\operatorname{Ker} \tau$.

Main example. Suppose $A^{*}$ is a commutative graded ring with identity, $1 \neq 0, G$ an arbitrary group, $H \subset G$ a subgroup of index $[G: H]=n$, and $1 / n ! \in A^{0}$. Suppose $G$ acts on $A^{*}$ by automorphisms. Then we have a tower of rings $i: A^{G} \hookrightarrow A^{H} \subset A^{*}$. In this case, the classical transfer $\tau: A^{H} \rightarrow A^{G}, \tau(a)=g_{1}(a)+g_{2}(a)+\ldots+g_{n}(a) \forall a \in A^{H}$ is defined, where $G=\left\{g_{1} H\right\} \sqcup\left\{g_{2} H\right\} \sqcup \ldots \sqcup\left\{g_{n} H\right\}$. We want to show that the classical group-theoretic transfer is an $n$-transfer in the sense of our definition.

First we show that $\tau: A^{H} \rightarrow A^{*}, \tau(a)=g_{1}(a)+\ldots+g_{n}(a) \forall a \in A^{H}$ is well-defined, i.e., $\tau$ is independent of the choice of the representatives $g_{i}$ from all cosets $\left\{g_{i} H\right\}_{1 \leq i \leq n}$. Suppose $g_{i}^{\prime}=g_{i} h_{i}, h_{i} \in H, 1 \leq i \leq n$ is another choice. We have $g_{i}^{\prime}(a)=g_{i}\left(h_{i}(a)\right)=g_{i}(a)$ $\forall a \in A^{H}, 1 \leq i \leq n$. Therefore $\left.g_{i}^{\prime}\right|_{A^{H}}=\left.g_{i}\right|_{A^{H}} \forall g_{i}^{\prime} \in\left\{g_{i} H\right\}, 1 \leq i \leq n$. Next we prove that $\operatorname{Im} \tau \subset A^{G}$. Pick arbitrary elements $a \in A^{H}, g \in G$. We have $g\left(g_{1}(a)+\ldots+g_{n}(a)\right)=$ $g g_{1}(a)+\ldots+g g_{n}(a)$. We need to show that the cosets $\left\{g g_{i} H\right\}_{1 \leq i \leq n}$ are distinct (and therefore $g g_{i}=g_{\sigma(i)}^{\prime},\left\{g_{\sigma(i)}^{\prime} H\right\}=\left\{g_{\sigma(i)} H\right\}, 1 \leq i \leq n$, for some permutation $\left.\sigma \in S_{n}\right)$. Indeed, if $\left\{g g_{i} H\right\}=\left\{g g_{j} H\right\}$ for some $i \neq j$, then $g g_{i}=g g_{j} h$ and $g_{i}=g_{j} h$ for some $h \in H$, which cannot happen.

Thus, $g(\tau(a))=g_{\sigma(1)}^{\prime}(a)+\ldots+g_{\sigma(n)}^{\prime}(a)=\tau(a) \forall a \in A^{H}, \forall g \in G$. Therefore, $\operatorname{Im} \tau \subset A^{G}$. But for any $a \in A^{G}$, we obviously have $\tau(a)=g_{1}(a)+\ldots+g_{n}(a)=n a$. Whence $\tau(a / n)=a \forall a \in A^{G}$. Therefore $\operatorname{Im} \tau=A^{G}$. We have checked that the group-theoretic transfer is well-defined. Now we need to show that $\tau: A^{H} \rightarrow A^{G}$ is an $n$-transfer in the sense of our definition. For any $\alpha \in A^{G}$ and $a \in A^{H}$, we have $\tau(\alpha a)=g_{1}(\alpha a)+\ldots+g_{n}(\alpha a)=g_{1}(\alpha) g_{1}(a)+\ldots+g_{n}(\alpha) g_{n}(a)=\alpha \tau(a)$. Therefore, $\tau: A^{H} \rightarrow A^{G}$ is an $A^{G}$-linear map of degree zero. Since the composition of $\tau: A^{H} \rightarrow A^{G}$ and the embedding $A^{G} \subset A^{*}$ splits into a sum of $n$ algebra homomorphisms $\tau=g_{1}+\ldots+$ $g_{n}: A^{H} \rightarrow A^{*}$, the map $\tau$ is an $n$-homomorphism. Choosing the identity $e \in G$ for $g_{1}$, we have $\tau(a)=a+\left(g_{2}(a)+\ldots+g_{n}(a)\right)=\operatorname{Id}(a)+g(a) \forall a \in A^{H}$, where $g: A^{H} \rightarrow A^{H} \subset A^{*}$, and $g=g_{2}+\ldots+g_{n}$ is, by virtue of splitting, an $A^{G}$-linear $(n-1)$-homomorphism.

Lemma 2.1.3. Suppose $A^{*}$ is a commutative $R^{*}$-algebra and $1 / n ! \in R^{0}$. Suppose $f: A^{*} \rightarrow A^{*}$ is an $n$-homomorphism and let $a_{1}, \ldots, a_{n+1} \in A^{*}$ be homogeneous elements such that

$$
f\left(a_{i_{1}} a_{i_{2}} \ldots a_{i_{k}}\right)=-a_{i_{1}} a_{i_{2}} \ldots a_{i_{k}} \quad \forall 1 \leq k \leq n, \forall 1 \leq i_{1}<i_{2}<\ldots<i_{k} \leq n+1 .
$$

Then $f\left(a_{1} a_{2} \ldots a_{n+1}\right)=n a_{1} a_{2} \ldots a_{n+1}$.

Proof. Suppose $A^{*}$ is a commutative $R^{*}$-algebra, $1 / n ! \in R^{0}, f: A^{*} \rightarrow A^{*}$ is an $n$ homomorphism, $a_{1}, \ldots, a_{n+1} \in A^{*}$ are homogeneous elements, and $\mu \in R^{0}$ is a scalar such that $f\left(a_{i_{1}} a_{i_{2}} \ldots a_{i_{k}}\right)=\mu a_{i_{1}} a_{i_{2}} \ldots a_{i_{k}} \forall 1 \leq k \leq n, \forall 1 \leq i_{1}<i_{2}<\ldots<i_{k} \leq n+1$. Since the algebra $A^{*}$ is commutative, it follows that for any pairwise distinct indices $\left\{i_{1}, \ldots, i_{k}\right\} \subset\{1,2, \ldots, n+1\} \forall 1 \leq k \leq n$ (in a not necessarily increasing order) we still have $f\left(a_{i_{1}} a_{i_{2}} \ldots a_{i_{k}}\right)=\mu a_{i_{1}} a_{i_{2}} \ldots a_{i_{k}}$. 
For any permutation $\sigma \in S_{n+1}$, let $\operatorname{NumCyc}(\sigma)$ denote the number of nonintersecting cycles in the decomposition of $\sigma$, including cycles of length one.

Expanding the graded Frobenius recursion at the $(n+1)$-st step, we have

$$
\begin{aligned}
& \Phi_{n+1}(f)\left(a_{1}, \ldots, a_{n+1}\right)=\sum_{\sigma \in S_{n+1}}(-1)^{\sigma}(-1)^{\sum_{p, q} \varepsilon^{p q} \varepsilon_{i-1(p) i}-1(q)}\left|a_{p} \| a_{q}\right|
\end{aligned}
$$

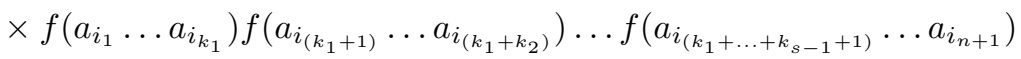

$$
\begin{aligned}
& =\sum_{\sigma \in S_{n+1}, \operatorname{NumCyc}(\sigma)=1}(\ldots)+\sum_{\sigma \in S_{n+1}, \operatorname{NumCyc}(\sigma) \geq 2}(\ldots)
\end{aligned}
$$

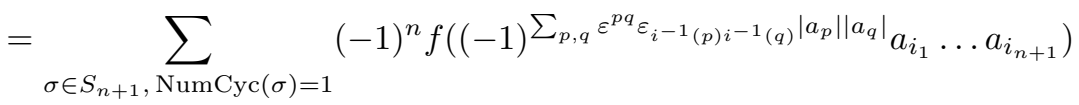

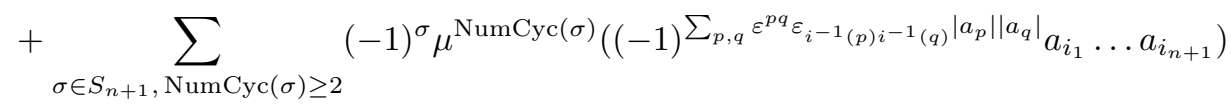

$$
\begin{aligned}
& =n !(-1)^{n} f\left(a_{1} \ldots a_{n+1}\right)+\left(\sum_{\sigma \in S_{n+1}, \operatorname{NumCyc}(\sigma) \geq 2}(-1)^{\sigma} \mu^{\mathrm{NumCyc}(\sigma)}\right) a_{1} \ldots a_{n+1} \text {. }
\end{aligned}
$$

The last equality follows from Lemma 1.1.2. Since $\Phi_{n+1}(f)\left(a_{1}, \ldots, a_{n+1}\right)=0$ and $1 / n ! \in$ $R^{0}$, we have $f\left(a_{1} a_{2} \ldots a_{n+1}\right)=\left(c_{2} \mu^{2}+\ldots+c_{n+1} \mu^{n+1}\right) a_{1} \ldots a_{n+1}$, where the $c_{i}$ are rather explicit rational constants of the form $N / n !, N \in \mathbb{Z}$.

Assume now that $f\left(a_{1} \ldots a_{n+1}\right)=\mu a_{1} \ldots a_{n+1}$. Then $\left(\mu-c_{2} \mu^{2}-\ldots-c_{n+1} \mu^{n+1}\right) a_{1} \ldots$ $a_{n+1}=0$. Denote the polynomial $\mu-c_{2} \mu^{2}-\ldots-c_{n+1} \mu^{n+1}$ by $q(\mu)$. First, we consider the case $R^{*}=\mathbb{C}$ and construct a commutative nongraded algebra $A$ over $\mathbb{C}$, an $n$ homomorphism $f: A \rightarrow A$, and a nonzero idempotent $a \in A, a^{2}=a, a \neq 0$ such that $f(a)=k a$, where $k$ is any of the numbers $\{1,2, \ldots, n\}$.

For $A$ we choose the algebra $A=C\left(X_{n}\right)$ of $\mathbb{C}$-valued functions on an $n$-element set $X_{n}=\{1, \ldots, n\}$. Clearly, this is the algebra of continuous $\mathbb{C}$-valued functions on the compact Hausdorff space $X_{n}$.

Suppose $X$ and $Y$ are compact Hausdorff spaces, and $C(X)$ and $C(Y)$ are the algebras of all continuous $\mathbb{C}$-valued functions on them. V. M. Buchstaber and E. G. Rees showed in [8] that any continuous $n$-homomorphism $\varphi: C(X) \rightarrow C(Y)$ is given by a uniquely determined continuous map $F^{\varphi}: Y \rightarrow \operatorname{Sym}^{n} X$ according to the formula

$$
\varphi(f)(y)=f\left(F_{1}^{\varphi}(y)\right)+f\left(F_{2}^{\varphi}(y)\right)+\ldots+f\left(F_{n}^{\varphi}(y)\right) \quad \forall f \in C(X), \forall y \in Y,
$$

where $\operatorname{Sym}^{n} X:=X^{n} / S_{n}$ is the $n$-th symmetric power of $X$ and the $n$-element $F^{\varphi}(y) \in$ $\operatorname{Sym}^{n} X$ is denoted by $\left[F_{1}^{\varphi}(y), \ldots, F_{n}^{\varphi}(y)\right]$.

By this result of V. M. Buchstaber and E. G. Rees, any $n$-homomorphism $f: C\left(X_{n}\right) \rightarrow$ $C\left(X_{n}\right)$ can be given by a map $F: X_{n} \rightarrow \operatorname{Sym}^{n} X_{n}$. Now fix an arbitrary natural $k$, $1 \leq k \leq n$ and define a map $F: X_{n} \rightarrow \operatorname{Sym}^{n} X_{n}: F(l)=\{1, \ldots, n\}=X_{n} \forall 1 \leq l \leq k$, $F(l)=n \cdot\{n\} \forall k<l \leq n$. The algebra $C\left(X_{n}\right)$ is nothing but the algebra $\bar{C}^{n}$ with a coordinate-wise multiplication. Consider the following idempotent $a \in \mathbb{C}^{n}$, where $a=(1, \ldots, 1,0, \ldots, 0)$, with first $k$ components equal to 1 . It is clear that $f(a)=$ $(k, \ldots, k, 0, \ldots, 0)=k a$. Now set $a_{1}=a_{2}=\ldots=a_{n+1}=a$, where $a$ is the above idempotent. It is easy to see that $f\left(a_{i_{1}} \ldots a_{i_{s}}\right)=k a_{i_{1}} \ldots a_{i_{s}} \forall 1 \leq s \leq n+1, \forall i_{j} \in$ $\{1, \ldots, n+1\}$. Therefore, we are in an already considered situation, and $q(k) a=0$, whence $q(k)=0$.

Thus, we have shown that the $n$ numbers $\{1, \ldots, n\}$ are roots of the polynomial $q(\mu)=\mu-c_{2} \mu^{2}-\ldots-c_{n+1} \mu^{n+1}$. It is clear that $\mu=0$ is also a root of $q(\mu)$. Since the degree of $q(\mu)$ is at most $n+1$ and the first nonzero monomial equals $\mu$, we have an 
explicit formula for $q(\mu)$ :

$$
q(\mu)=(-1)^{n} \frac{1}{n !} \mu(\mu-1) \ldots(\mu-n) .
$$

Suppose now that the conditions of the lemma hold; i.e., we have an $n$-homomorphism $f: A^{*} \rightarrow A^{*}$ of a commutative $R^{*}$-algebra $A^{*}, 1 / n ! \in R^{0}$, and homogeneous elements $a_{1}, \ldots, a_{n+1} \in A^{*}$ such that $f\left(a_{i_{1}} \ldots a_{i_{k}}\right)=-a_{i_{1}} \ldots a_{i_{k}} \forall 1 \leq k \leq n, \forall 1 \leq i_{1}<i_{2}<$ $\ldots<i_{k} \leq n+1$. Then, by the above,

$$
f\left(a_{1} \ldots a_{n+1}\right)=\left(c_{2}(-1)^{2}+\ldots+c_{n+1}(-1)^{n+1}\right) a_{1} a_{2} \ldots a_{n+1} .
$$

It is easy to see that

$$
\begin{aligned}
c_{2}(-1)^{2}+\ldots+c_{n+1}(-1)^{n+1} & =-q(-1)-1 \\
& =(-1)^{n} \frac{1}{n !}(-1)(-2) \ldots(-(n+1))-1=n .
\end{aligned}
$$

Thus, $f\left(a_{1} \ldots a_{n+1}\right)=n a_{1} a_{2} \ldots a_{n+1}$, which proves the lemma.

Lemma 2.1.4. Suppose $A^{*}$ and $B^{*}$ are $R^{*}$-algebras, $B^{*}$ is commutative, $I^{*} \subset A^{*}$ is a homogenous two-sided ideal, $J^{*} \subset B^{*}$ a homogenous ideal, and $f: A^{*} \rightarrow B^{*}$ is an $n$-homomorphism such that $f\left(I^{*}\right) \subset J^{*}$. Then the induced $R^{*}$-linear map $\bar{f}: A^{*} / I^{*} \rightarrow$ $B^{*} / J^{*}$ is also an $n$-homomorphism of algebras.

Proof. We shall verify all conditions from the definition of an $n$-homomorphism. For arbitrary homogeneous elements $\bar{a}_{1}, \bar{a}_{2} \in A^{*} / I^{*}$, choose their representatives $a_{1}, a_{2} \in A^{*}$, $\pi_{I^{*}}\left(a_{1}\right)=\bar{a}_{1}, \pi_{I^{*}}\left(a_{2}\right)=\bar{a}_{2}$. Then

$$
\begin{aligned}
\bar{f}\left(\bar{a}_{1} \bar{a}_{2}\right) & =\pi_{J^{*}}\left(f\left(a_{1} a_{2}\right)\right)=\pi_{J^{*}}\left((-1)^{\left|a_{1}\right|\left|a_{2}\right|} f\left(a_{2} a_{1}\right)\right) \\
& =(-1)^{\left|\bar{a}_{1}\right|\left|\bar{a}_{2}\right|} \pi_{J^{*}}\left(f\left(a_{2} a_{1}\right)\right)=(-1)^{\left|\bar{a}_{1}\right|\left|\bar{a}_{2}\right|} \bar{f}\left(\bar{a}_{2} \bar{a}_{1}\right),
\end{aligned}
$$

and condition (1) holds.

(2) $\bar{f}\left(1_{A^{*} / I^{*}}\right)=\pi_{J^{*}}\left(f\left(1_{A^{*}}\right)\right)=\pi_{J^{*}}\left(n \cdot 1_{B^{*}}\right)=n \cdot 1_{B^{*} / J^{*}}$.

(3) For arbitrary homogeneous elements $\bar{a}_{1}, \ldots, \bar{a}_{n+1} \in A^{*} / I^{*}$, choose their representatives $a_{1}, \ldots, a_{n+1} \in A^{*}, \pi_{I^{*}}\left(a_{i}\right)=\bar{a}_{i} \forall 1 \leq i \leq n+1$. It is easy to see that $\Phi_{n+1}(\bar{f})\left(\bar{a}_{1}, \ldots, \bar{a}_{n+1}\right)=\pi_{J^{*}}\left(\Phi_{n+1}(f)\left(a_{1}, \ldots, a_{n+1}\right)\right)=\pi_{J^{*}}(0)=0$. Thus condition $(3)$ also holds.

Proposition 2.1.5. Suppose $i: B^{*} \rightarrow A^{*}, \tau: A^{*} \rightarrow B^{*}$ is an n-transfer. Suppose also that $I^{*} \subset B^{*}$ is a homogeneous ideal, $J^{*}=A^{*} I^{*}$ is its extension to $A^{*}$, and $a_{1}, a_{2}, \ldots, a_{n} \in A^{*}$ are homogeneous elements such that

$$
\tau\left(a_{i_{1}} a_{i_{2}} \ldots a_{i_{k}}\right) \in I^{*} \quad \forall 1 \leq k \leq n, \forall 1 \leq i_{1}<i_{2}<\ldots<i_{k} \leq n .
$$

Then $a_{1} a_{2} \ldots a_{n} \in J^{*}$.

Proof. Consider the ideal $J^{*} \cap B^{*} \subset B^{*}$ and the following commutative diagram:

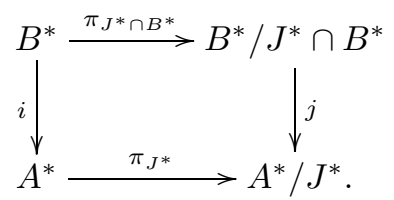

It is clear that the algebra homomorphism $j: B^{*} / J^{*} \cap B^{*} \rightarrow A^{*} / J^{*}$ is a monomorphism. For any $a \in J^{*}$ we have $a=\sum_{i=1}^{N} b_{i} a_{i}, b_{i} \in I^{*}, 1 \leq i \leq N$. As $\tau$ is $B^{*}$-linear, we have $\tau(a)=\sum_{i=1}^{N} b_{i} \tau\left(a_{i}\right) \in I^{*} \subset J^{*} \cap B^{*}$. Thus, $\tau\left(J^{*}\right) \subset J^{*} \cap B^{*}$.

By Lemma 2.1.4, the induced map $\bar{\tau}: A^{*} / J^{*} \rightarrow B^{*} / J^{*} \cap B^{*}$ is a $B^{*}$-linear $n$-homomorphism. Consider arbitrary elements $\bar{b} \in B^{*} / J^{*} \cap B^{*}$ and $\bar{a} \in A^{*} / J^{*}$, and choose their 
representatives $b \in B^{*}, \pi_{J^{*} \cap B^{*}}(b)=\bar{b}$ and $a \in A^{*}$ and $\pi_{J^{*}}(a)=\bar{a}$. Then $\bar{\tau}(\bar{b} \bar{a})=$ $\bar{\tau}(j(\bar{b}) \bar{a})=\pi_{J^{*} \cap B^{*}}(\tau(i(b) a))=\pi_{J^{*} \cap B^{*}}(b \tau(a))=\bar{b} \bar{\tau}(\bar{a})$. Thus, the map $\bar{\tau}: A^{*} / J^{*} \rightarrow$ $B^{*} / J^{*} \cap B^{*}$ is a $\left(B^{*} / J^{*} \cap B^{*}\right)$-linear $n$-homomorphism.

For the original $n$-transfer $\tau: A^{*} \rightarrow B^{*}$, we had $i \circ \tau(a)=a+g(a) \forall a \in A^{*}$, where $g: A^{*} \rightarrow A^{*}$ is a uniquely determined $(n-1)$-homomorphism. Consider an arbitrary element $a \in J^{*}$. As was proved above, $\tau(a) \in J^{*} \cap B^{*}$. Therefore, $i \circ \tau(a) \in J^{*}$, and thus $g(a)=i \circ \tau(a)-a \in J^{*} \forall a \in J^{*}$, i.e., $g\left(J^{*}\right) \subset J^{*}$. By Lemma 2.1.4, the induced map $\bar{g}: A^{*} / J^{*} \rightarrow A^{*} / J^{*}$ is an $(n-1)$-homomorphism. Passing to the quotient of $i \circ \tau=\operatorname{Id}_{A^{*}}+g$ by the ideal $J^{*}$, we have

$$
j \circ \bar{\tau}=\operatorname{Id}_{A^{*} / J^{*}}+\bar{g} .
$$

In summary, the constructed map $\bar{\tau}: A^{*} / J^{*} \rightarrow B^{*} / J^{*} \cap B^{*}$ is an $n$-transfer with respect to the inclusion $j: B^{*} / J^{*} \cap B^{*} \rightarrow A^{*} / J^{*}$. Suppose now that $a_{1}, \ldots, a_{n} \in A^{*}$ are homogeneous elements such that $\tau\left(a_{i_{1}} \ldots a_{i_{k}}\right) \in I^{*} \forall 1 \leq k \leq n, \forall 1 \leq i_{1}<i_{2}<\ldots<$ $i_{k} \leq n$. We want to show that $a_{1} a_{2} \ldots a_{n} \in J^{*}$.

Set $\pi_{J^{*}}\left(a_{s}\right)=\bar{a}_{s} \in A^{*} / J^{*}, 1 \leq s \leq n$. Since $\tau\left(a_{i_{1}} \ldots a_{i_{k}}\right) \in I^{*} \subset J^{*} \cap B^{*}$, we have $\bar{\tau}\left(\bar{a}_{i_{1}} \ldots \bar{a}_{i_{k}}\right)=0$. Since $j \circ \bar{\tau}=\operatorname{Id}_{A^{*} / J^{*}}+\bar{g}$, we also have $\bar{g}\left(\bar{a}_{i_{1}} \ldots \bar{a}_{i_{k}}\right)=-\bar{a}_{i_{1}} \ldots \bar{a}_{i_{k}}$ $\forall 1 \leq k \leq n, \forall 1 \leq i_{1}<i_{2}<\ldots<i_{k} \leq n$, where $\bar{g}: A^{*} / J^{*} \rightarrow A^{*} / J^{*}$ is the $(n-1)$ homomorphism constructed earlier. It now follows from Lemma 2.1.3 that $\bar{g}\left(\bar{a}_{1} \ldots \bar{a}_{n}\right)=$ $(n-1) \bar{a}_{1} \ldots \bar{a}_{n}$. But we also have $\bar{g}\left(\bar{a}_{1} \ldots \bar{a}_{n}\right)=-\bar{a}_{1} \ldots \bar{a}_{n}$. Thus, $n \bar{a}_{1} \ldots \bar{a}_{n}=0$. Since $1 / n ! \in A^{0}$ and $A^{*} / J^{*}$ is an $A^{*}$-algebra, we have $0=\bar{a}_{1} \ldots \bar{a}_{n}=\pi_{J^{*}}\left(a_{1} \ldots a_{n}\right)$, and, therefore, $a_{1} \ldots a_{n} \in J^{*}$.

We define the multiplicative length $l\left(A^{*}\right)$ of a graded commutative ring $A^{*}$ to be the largest integer $m$ for which there exist homogeneous elements $a_{1}, a_{2}, \ldots, a_{m} \in A^{* \geq 1}$ of positive degrees such that $a_{1} a_{2} \ldots a_{m} \neq 0$ (if such elements exist for any $m$, we set $\left.l\left(A^{*}\right)=\infty\right)$. It is clear that $l\left(A^{*}\right)=0$ if and only if $A^{q}=\{0\} \forall q \geq 1$.

Theorem 2.1.6. Suppose $i: B^{*} \rightarrow A^{*}, \tau: A^{*} \rightarrow B^{*}$ is an $n$-transfer. Then we have a sharp bound

$$
l\left(B^{*}\right)+1 \geq \frac{l\left(A^{*}\right)+1}{n} .
$$

The equality happens, for example, in the case of a classical transfer $i: A^{\mathbb{Z}_{n}} \rightarrow A^{*}$, $\tau: A^{*} \rightarrow A^{\mathbb{Z}_{n}}$, where for $A^{*}$ we can choose a finite-dimensional connected graded algebra over $\mathbb{Q}$ with an action of a cyclic group $\mathbb{Z}_{n}$ with any fixed $n \geq 2$ and $l\left(A^{*}\right) \geq 0$.

Proof. Consider the ideal $I^{*}=B^{* \geq 1}$ of $B^{*}$ generated by the homogeneous elements of positive degrees. Then, for any homogeneous elements $a_{1}, \ldots, a_{n} \in A^{*}$ of positive degrees $\left|a_{i}\right| \geq 1,1 \leq i \leq n$, we have $\tau\left(a_{i_{1}} \ldots a_{i_{k}}\right) \in B^{* \geq 1}=I^{*} \forall 1 \leq k \leq n, \forall 1 \leq i_{1}<i_{2}<$ $\ldots<i_{k} \leq n$. Therefore, by Proposition 2.1.5, we have $a_{1} \ldots a_{n} \in \bar{A}^{*} I^{*}=\bar{A}^{*} B^{*} \geq 1$. In other words, $a_{1} \ldots a_{n}=\sum_{i=1}^{N} b_{i} a_{i}^{\prime}$, for some $b_{i} \in B^{*},\left|b_{i}\right| \geq 1,1 \leq i \leq N$. Consider now $\left(l\left(B^{*}\right)+1\right) n$ arbitrary homogeneous elements $a_{1}, \ldots, a_{\left(l\left(B^{*}\right)+1\right) n} \in A^{*}$ of positive degrees, and write their product in the form $a_{1} \ldots a_{\left(l\left(B^{*}\right)+1\right) n}=\prod_{0 \leq k \leq l\left(B^{*}\right)}\left(a_{n k+1} a_{n k+2} \ldots a_{n k+n}\right)$. Each factor $a_{n k+1} a_{n k+2} \ldots a_{n k+n}, 0 \leq k \leq l\left(B^{*}\right)$, as we saw before, becomes a finite sum of the products $b_{i} a_{i}^{\prime}$, where $\left|b_{i}\right| \geq 1$. Therefore, the entire product $a_{1} \ldots a_{\left(l\left(B^{*}\right)+1\right) n}$ breaks into a finite sum, all of whose summands contain a product of $l\left(B^{*}\right)+1$ elements from $B^{* \geq 1}$, and, therefore, each such summand is zero. Therefore, the entire product $a_{1} \ldots a_{\left(l\left(B^{*}\right)+1\right) n}$ is zero.

This yields the desired bound

$$
n\left(l\left(B^{*}\right)+1\right) \geq l\left(A^{*}\right)+1, \quad l\left(B^{*}\right)+1 \geq \frac{l\left(A^{*}\right)+1}{n} .
$$


Now fix arbitrary $N \geq 0$ and $n \geq 2$. We shall construct connected finite-dimensional $\mathbb{Q}$-algebras $A_{N}^{*}$ with an action of the cyclic group $\mathbb{Z}_{n}$ such that $l\left(A_{N}^{*}\right)=N$ and $l\left(A^{\mathbb{Z}_{n}}\right)+1$ is the smallest integer greater than or equal to $\frac{l\left(A_{N}^{*}\right)+1}{n}=\frac{N+1}{n}$. This would show that the obtained estimate is sharp. Consider the cyclotomic field $K_{n}=\mathbb{Q}\left(e^{2 \pi i / n}\right)$, i.e., the splitting field of $x^{n}-1$ over $\mathbb{Q}$. As is known, $K_{n} \supset \mathbb{Q}$ is a finite extension of $\mathbb{Q}$ and $\operatorname{dim}_{\mathbb{Q}} K_{n}=\varphi(n)$, where $\varphi(n)$ is the Euler function. Let $\tilde{A}_{N}^{*}=\Lambda_{K_{n}}\left(\alpha_{1}, \ldots, \alpha_{N}\right)$ be the exterior algebra on $N$ degree-one formal generators $\alpha_{1}, \ldots, \alpha_{N}$ over $K_{n}$. It is clear that $\tilde{A}_{N}^{*}$ is a connected finite-dimensional algebra over $K_{n}$, but since $K_{n}$ contains $\mathbb{Q}$ and $\operatorname{dim}_{\mathbb{Q}} K_{n}=\varphi(n)<\infty, \tilde{A}_{N}^{*}$ is also a finite-dimensional algebra over $\mathbb{Q}$. Now we need an auxiliary construction.

Suppose $A^{*}$ is a commutative graded algebra with identity over a nongraded ring $R$, and $\lambda \in R$ is an invertible scalar. Define a map $\varphi_{\lambda}: A^{*} \rightarrow A^{*}$ by setting $\varphi_{\lambda}(a)=\lambda^{|a|} a$. First we check that it is $R$-linear. By definition, $\varphi_{\lambda}\left(A^{i}\right) \subset A^{i}$. If $a, a^{\prime} \in A^{i}, i \in \mathbb{Z}_{+}$, then

$$
\varphi_{\lambda}\left(a+a^{\prime}\right)=\lambda^{i}\left(a+a^{\prime}\right)=\lambda^{i} a+\lambda^{i} a^{\prime}=\varphi_{\lambda}(a)+\varphi_{\lambda}\left(a^{\prime}\right) .
$$

Furthermore, $\varphi_{\lambda}(\mu a)=\lambda^{|\mu a|}(\mu a)=\lambda^{|a|} \mu a=\mu\left(\lambda^{|a|} a\right)=\mu \varphi_{\lambda}(a)$. Finally,

$$
\begin{gathered}
\varphi_{\lambda}(1)=\lambda^{0} \cdot 1=1 \\
\varphi_{\lambda}(a b)=\lambda^{|a b|} a b=\lambda^{|a|} \lambda^{|b|} a b=\left(\lambda^{|a|} a\right)\left(\lambda^{|b|} b\right)=\varphi_{\lambda}(a) \varphi_{\lambda}(b)
\end{gathered}
$$

$\forall a \in A^{i}, i \in \mathbb{Z}_{+}, \forall b \in A^{j}, j \in \mathbb{Z}_{+}$. Thus, we have shown that $\varphi_{\lambda}: A^{*} \rightarrow A^{*}$ is an algebra endomorphism. But $\varphi_{\lambda} \circ \varphi_{\lambda^{-1}}=\varphi_{1}=\operatorname{Id}_{A^{*}}, \varphi_{\lambda^{-1}} \circ \varphi_{\lambda}=\varphi_{1}=\operatorname{Id}_{A^{*}}$. Therefore, for any invertible scalar $\lambda \in R$, the map $\varphi_{\lambda}: A^{*} \rightarrow A^{*}$ is an automorphism.

Now we return to the cyclotomic field $K_{n}=\mathbb{Q}\left(e^{2 \pi i / n}\right)$ and the algebra $\tilde{A}_{N}^{*}$. Set $\varepsilon=e^{2 \pi i / n} \in K_{n}$, a primitive $n$-th root of unity. By the just described construction, we have the automorphism $\varphi_{\varepsilon}: \tilde{A}_{N}^{*} \rightarrow \tilde{A}_{N}^{*}$, corresponding to the invertible scalar $\varepsilon \in K_{n}$. Clearly, $\varphi_{\varepsilon}^{n}=\varphi_{\varepsilon^{n}}=\varphi_{1}=\operatorname{Id}_{\tilde{A}_{N}^{*}}$. Therefore, the automorphism $\varphi_{\varepsilon}$ gives rise to an action of the cyclic group $\mathbb{Z}_{n}$. Now we want to determine the invariant subalgebra $\tilde{A}_{N}^{\mathbb{Z}_{n}}$. Since $\varphi_{\varepsilon}(a)=\varepsilon^{i} a \forall a \in A^{i}, \forall i \geq 0$, the invariant homogeneous elements are precisely those whose degrees are divisible by $n$. In other words, $\tilde{A}_{N}^{\mathbb{Z}_{n}}=K_{n} \oplus \tilde{A}_{N}^{n} \oplus \tilde{A}_{N}^{2 n} \oplus \ldots$.

It is clear that $l\left(\tilde{A}_{N}^{*}\right)=N$. To determine the multiplicative length $l\left(\tilde{A}_{N}^{\mathbb{Z}_{n}}\right)$, suppose $N / n=s+r / n, s \geq 0,0 \leq r<n$ and set $m=l\left(\tilde{A}_{N}^{\mathbb{Z}_{n}}\right)$. Suppose $a_{1}, \ldots, a_{m} \in \tilde{A}_{N}^{\mathbb{Z}_{n}}$ are elements of degrees $\left|a_{j}\right|=n k_{j}, k_{j} \in \mathbb{N}, 1 \leq j \leq m$ such that $a_{1} \ldots a_{m} \neq 0$. It is not difficult to check that for $a_{1}, \ldots, a_{m}$ we can choose exterior monomials of the form $\alpha_{i_{1}} \wedge \ldots \wedge \alpha_{i_{n k_{j}}}, 1 \leq i_{1}<\ldots<i_{n k_{j}} \leq N, 1 \leq j \leq m$. Since $m$ is maximal, it follows that all $a_{1}, \ldots, a_{m}$ are in $\tilde{A}_{N}^{n}$. It is now quite easy to check that $m=l\left(\tilde{A}_{N}^{\mathbb{Z}_{n}}\right)=s$. Our estimate

$$
l\left(\tilde{A}_{N}^{\mathbb{Z}_{n}}\right)+1 \geq \frac{l\left(\tilde{A}_{N}^{*}\right)+1}{n}
$$

becomes $s+1 \geq \frac{N+1}{n}=s+\frac{r+1}{n}, 1 \leq r+1 \leq n$, and we see that $l\left(\tilde{A}_{N}^{\mathbb{Z}_{n}}\right)+1$ is the smallest of the integers which are greater than or equal to $\frac{l\left(\tilde{A}_{N}^{*}\right)+1}{n}$.

Thus, over the cyclotomic field $K_{n}$ we have constructed connected finite-dimensional algebras $\tilde{A}_{N}^{*}, l\left(\tilde{A}_{N}^{*}\right)=N$, with an action of the cyclic group $\mathbb{Z}_{n}$, for which the estimate $l\left(\tilde{A}_{N}^{\mathbb{Z}_{n}}\right)+1 \geq \frac{l\left(\tilde{A}_{N}^{*}\right)+1}{n}$ is sharp. But since $K_{n} \supset \mathbb{Q}$ is a finite extension of $\mathbb{Q}$, we can view $K_{n}$-algebras $\tilde{A}_{N}^{*}$ as finite-dimensional algebras over $\mathbb{Q}$. We denote them by $A_{N}^{\prime *}$ and notice that the action of the cyclic group by $K_{n}$-linear automorphisms on $\tilde{A}_{N}^{*}$ is, of course, an action by $\mathbb{Q}$-linear automorphisms on $A_{N}^{\prime *}$. Finally, to obtain a connected $\mathbb{Q}$-algebra, consider the following subalgebra $A_{N}^{*}$ of $A_{N}^{\prime *}$ :

$$
A_{N}^{0}=\mathbb{Q} \subset K_{n}=A_{N}^{\prime 0}, \quad A_{N}^{i}=A_{N}^{\prime i} \quad \forall i>0 .
$$


It is clear that we have isomorphisms $A_{N}^{* \geq 1} \cong A_{N}^{* \geq 1} \cong \tilde{A}_{N}^{* \geq 1}$ and $\left(A_{N}^{\mathbb{Z}_{n}}\right)^{* \geq 1} \cong$ $\left(A_{N}^{\prime \mathbb{Z}_{n}}\right)^{* \geq 1} \cong\left(\tilde{A}_{N}^{\mathbb{Z}_{n}}\right)^{* \geq 1}$ of graded rings. Therefore, $l\left(A_{N}^{*}\right)=l\left(\tilde{A}_{N}^{*}\right)=N$ and $l\left(A_{N}^{\mathbb{Z}_{n}}\right)=$ $l\left(\tilde{A}_{N}^{\mathbb{Z}_{n}}\right)$. Hence our estimate $l\left(A_{N}^{\mathbb{Z}_{n}}\right)+1 \geq \frac{l\left(A_{N}^{*}\right)+1}{n}$ for $A_{N}^{*}$ coincides with the estimate for $\tilde{A}_{N}^{*}$ and is therefore also sharp.

\subsection{Smith-Dold branched coverings.}

Definition 2.2.1. Suppose $X$ and $Y$ are Hausdorff spaces. A continuous map $f: X \rightarrow Y$ is called an $n$-fold branched covering if $f$ is surjective, open-closed, and for any point $y \in Y$ the set $f^{-1}(y)$ consists of no more than $n$ points.

Remark. Clearly, a 1-fold branched covering is just a homeomorphism. It is not difficult to show that a 2 -fold branched covering is always a quotient by an action of $\mathbb{Z}_{2}$.

Let $\exp _{n}(X)$ be the set of all nonempty subsets with at most $n$ elements in a Hausdorff space $X$, endowed with the standard Vietoris topology. Let $\operatorname{Sym}^{n} X=X^{n} / S_{n}$ be the $n$-th symmetric power of $X$ endowed with the quotient topology. Clearly, there is a canonical projection ("dropping the coefficients")

$$
\begin{gathered}
\langle\cdot\rangle: \operatorname{Sym}^{n} X \rightarrow \exp _{n}(X), \\
\left\langle\left[k_{1} x_{1}, \ldots, k_{s} x_{s}\right]\right\rangle=\left\{x_{1}, \ldots, x_{s}\right\}, \quad x_{i} \neq x_{j}, i \neq j, k_{1}+\ldots+k_{s}=n .
\end{gathered}
$$

It is also clear that $\operatorname{Sym}^{1} X=\exp _{1}(X)=X$ and $\operatorname{Sym}^{2} X \cong \exp _{2}(X)$.

Definition 2.2.2. Suppose $X$ and $Y$ are Hausdorff spaces. A continuous map $f: X \rightarrow$ $Y$ is called an $n$-fold Smith-Dold branched covering if there exists a continuous map $g: Y \rightarrow \operatorname{Sym}^{n} X$ such that $\langle g(y)\rangle=f^{-1}(y) \forall y \in Y$.

Branched coverings of this type were introduced by L. Smith 28] in 1983 as a generalization of regular coverings, which allowed an extension of (co)homological transfer. In 1986, A. Dold [17] gave a complete classification of branched coverings, introduced by L. Smith, in terms of actions of finite groups on topological spaces. Later on, maps of this type were called Smith-Dold branched coverings. The following key result of A. Dold [17] will be of fundamental importance to us.

Theorem. (i) Suppose $X$ is a Hausdorff space, $G$ a finite group acting on $X$, and $H \subset G$ is a subgroup of index $n$. Then the canonical projection $\pi_{G, H}: X / H \rightarrow X / G$ is an $n$-fold Smith-Dold branched covering.

(ii) Suppose $X$ and $Y$ are Hausdorff spaces, and $f: X \rightarrow Y, g: Y \rightarrow \operatorname{Sym}^{n} X$ is an $n$-fold Smith-Dold branched covering. Then there exists a canonical Hausdorff space $W$ with an $S_{n}$-action such that $X=W / S_{n-1}, Y=W / S_{n}$ and the map $f: X \rightarrow Y$ coincides with the map $\pi_{S_{n}, S_{n-1}}: W / S_{n-1} \rightarrow W / S_{n}$.

It is not difficult to check that if, in Definition 2.2.2, $\operatorname{Sym}^{n} X$ is replaced by $\exp _{n}(X)$, then we recover Definition 2.2.1. It now follows that for $n=1,2$, any branched covering is a Smith-Dold branched covering. However, for $n \geq 3$ the situation is completely different; namely, there are $n$-fold branched coverings (even those of a graph over another graph), which are not $N$-fold Smith-Dold branched coverings for any $N$.

We want to give an example of such a map. Let $X \subset S^{1} \times I^{1}$ be the union of the following three subsets of a cylinder:

$$
X_{1}=S^{1} \times\{0\}, \quad X_{2}=S^{1} \times\{1\}, \quad X_{3}=\left\{\left(e^{i \pi t}, t\right) \mid 0 \leq t \leq 1\right\} .
$$

For $Y$ we take the circle $Y=\left\{e^{i \varphi} \mid 0 \leq \varphi \leq 2 \pi\right\}$ and let $f: X \rightarrow Y$ be the projection $X \ni\left(e^{i \varphi}, t\right) \mapsto e^{i \varphi} \in Y$. It is clear that $f: X \rightarrow Y$ is surjective, at most 3-fold, and the complete preimage map $f^{-1}: Y \rightarrow \exp _{3}(X)$ is continuous. Thus, $f$ is a 3 -fold branched 
covering. We shall show that $f: X \rightarrow Y$ is not an $N$-fold Smith-Dold branched covering for any $N$.

Assume the opposite. Suppose $g: Y \rightarrow \operatorname{Sym}^{N} X$ is a continuous map such that $f^{-1}(y)=\langle g(y)\rangle \forall y \in Y$. Thus $g$ assigns a natural multiplicity to each point of the preimage of $y \in Y$ in a continuous way.

For any $e^{i \varphi} \in Y, 0<\varphi<2 \pi$, one of the points in the preimage is $\left(e^{i \varphi}, 0\right)$. The map $g: Y \rightarrow \operatorname{Sym}^{N} X$ assigns to $\left(e^{i \varphi}, 0\right), 0<\varphi<2 \pi$ some natural multiplicity $1 \leq K_{\varphi} \leq N$. Since $g$ is continuous, it is clear that $K_{\varphi}=$ const $=k \forall 0<\varphi<2 \pi$. We want to determine the possible values of the multiplicity $l$ of the point $(1,0) \in X$. On the one hand, $l$ has to be equal to $k$, because a little bit to the left from $1=e^{2 \pi i} \in Y$ we have $y_{-\varepsilon}=e^{(2 \pi-\varepsilon) i}$, and at the unique point of the preimage of $y_{-\varepsilon}$ near $(1,0)$ the multiplicity is $k$, which has to be $l$, the multiplicity of $(1,0)$. Thus, $l=k$. On the other hand, $l$ has to be equal to the sum of the multiplicities of all points of the preimage $y_{\varepsilon}=e^{\varepsilon i}$, $0<\varepsilon \ll 1$, close to $(1,0)$. But there are only two such points: $\left(e^{\varepsilon i}, 0\right)$ and $\left(e^{\varepsilon i}, \varepsilon / \pi\right)$. Therefore, $l$ equals $k+$ (multiplicity of $\left(e^{\varepsilon i}, \varepsilon / \pi\right)$ ). Thus, $l=k$ and $l \geq k+1$.

The obtained contradiction shows that the above 3 -fold branched covering $f: X \rightarrow Y$ of a graph over a circle is not an $N$-fold Smith-Dold branched covering for any $N$. This example can easily be modified to make the degree of the branched covering $f: X \rightarrow Y$ equal to any $n \geq 3$.

It is clear that any finite regular (nonbranched) covering $f: X \rightarrow Y$ of Hausdorff spaces is an $n$-fold Smith-Dold branched covering, where $n$ is the degree of $f: X \rightarrow Y$. Moreover, the map $g: Y \rightarrow \operatorname{Sym}^{n} X$ assigns to each point $y \in Y$ all $n$ points of its preimage $f^{-1}(y)$ with multiplicity one.

In the proof of the main result of the second section we shall use the following two wellknown theorems on cohomology of paracompact spaces with coefficients in a constant sheaf. They can both be found, for example, in [4].

Theorem A. Suppose $X$ is a paracompact space, $G$ a finite group acting on $X$, and $K$ a field of characteristic zero or $p$, with $(p,|G|)=1$. Let $\pi: X \rightarrow X / G$ be the canonical projection. Then the induced map $\pi^{*}: H_{\text {sheaf }}^{*}(X / G ; K) \rightarrow H_{\text {sheaf }}^{*}(X ; K)^{G}$ on the cohomology with coefficients in a constant sheaf is an isomorphism.

Theorem B. Suppose $X$ is a locally contractible paracompact space, and $R$ a ring which is a field $K$ or $\mathbb{Z}$. Then there exists a functorial isomorphism $H^{*}(X ; R) \rightarrow H_{\text {sheaf }}^{*}(X ; R)$ between the singular cohomology $R$-algebra $H^{*}(X ; R)$ of $X$ with coefficients in $R$ and the cohomology $R$-algebra $H_{\text {sheaf }}^{*}(X ; R)$ of $X$ with coefficients in a constant sheaf.

Let $l(X)$ be the rational cohomological length of $X$, i.e., the largest integer $m$ such that there are homogeneous elements $a_{1}, a_{2}, \ldots, a_{m} \in H^{* \geq 1}(X ; \mathbb{Q})$ of positive degrees such that $a_{1} a_{2} \ldots a_{m} \neq 0$. Similarly, let $l_{p}(X)$ be the $\mathbb{Z}_{p}$-cohomological length of $X$.

Now we are ready to state and prove the main result of the second section.

Theorem 2.2.3. Let $X$ and $Y$ be locally contractible paracompact spaces and $f: X \rightarrow Y$ an $n$-fold Smith-Dold branched covering. Then

$$
l(Y)+1 \geq \frac{l(X)+1}{n}, \quad l_{p}(Y)+1 \geq \frac{l_{p}(X)+1}{n} \quad \forall p>n .
$$

If $n=2$, these bounds are sharp.

Proof. We shall now show that there are $n$-transfers $\tau: H^{*}(X ; \mathbb{Q}) \rightarrow H^{*}(Y ; \mathbb{Q})$ and $\tau: H^{*}\left(X ; \mathbb{Z}_{p}\right) \rightarrow H^{*}\left(Y ; \mathbb{Z}_{p}\right), p>n$, in singular cohomology, corresponding to the homomorphisms $f^{*}: H^{*}(Y ; \mathbb{Q}) \rightarrow H^{*}(X ; \mathbb{Q})$ and $f^{*}: H^{*}\left(Y ; \mathbb{Z}_{p}\right) \rightarrow H^{*}\left(X ; \mathbb{Z}_{p}\right), p>n$. 
In the theorem of $\mathrm{A}$. Dold mentioned above, the space $W$ is the pullback

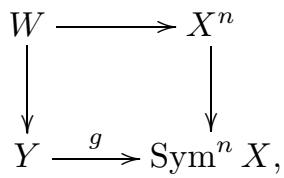

$W=\left\{\left(y, x_{1}, \ldots, x_{n}\right) \in Y \times X^{n} \mid g(y)=\left[x_{1}, \ldots, x_{n}\right]\right\}$. The group $S_{n}$ acts on $W$ as follows: $\sigma\left(y, x_{1}, \ldots, x_{n}\right)=\left(y, x_{\sigma(1)}, \ldots, x_{\sigma(n)}\right) \forall\left(y, x_{1}, \ldots, x_{n}\right) \in W, \forall \sigma \in S_{n}$. The space $W$ is obviously Hausdorff. Recall ([3, Ch. 1, Th. 3.1]) that the projection to the quotient by an action of a compact group is a closed proper (i.e., perfect) map. In particular, the map $\pi_{S_{n}}: W \rightarrow Y$ is perfect. The preimage of a paracompact space under a perfect map is always paracompact [21, p. 461, Th. 5.1.35]. It now follows that $W$ is paracompact.

By the theorem of A. Dold, we have the equality of continuous maps $(f: X \rightarrow Y)=$ $\left(\pi_{S_{n}, S_{n-1}}: W / S_{n-1} \rightarrow W / S_{n}\right)$. Since $X$ and $Y$ are locally contractible paracompact spaces, it follows from Theorem $B$ that their singular cohomology algebras with coefficients in the ring $R$ are canonically isomorphic to their cohomology algebras with coefficients in the constant sheaf $R$, where $R$ is a field or $\mathbb{Z}$.

Suppose $K$ is one of the fields $\mathbb{Q}$ or $\mathbb{Z}_{p}, p>n$. By the above arguments, the homomorphism $f^{*}: H^{*}(Y ; K) \rightarrow H^{*}(X ; K)$ of singular cohomology algebras can be identified with the homomorphism of the cohomology algebras with coefficients in a constant sheaf $\pi_{S_{n}, S_{n-1} \text { (sheaf) }}^{*}: H_{\text {sheaf }}^{*}\left(W / S_{n} ; K\right) \rightarrow H_{\text {sheaf }}^{*}\left(W / S_{n-1} ; K\right)$.

By Theorem $A$, we have isomorphisms

$$
\begin{gathered}
\pi_{\left.S_{n} \text { (sheaf) }\right)}^{*}: H_{\text {sheaf }}^{*}\left(W / S_{n} ; K\right) \cong H_{\text {sheaf }}^{*}(W ; K)^{S_{n}} ; \\
\pi_{S_{n-1}(\text { sheaf })}^{*}: H_{\text {sheaf }}^{*}\left(W / S_{n-1} ; K\right) \cong H_{\text {sheaf }}^{*}(W ; K)^{S_{n-1}} .
\end{gathered}
$$

Since $\pi_{S_{n}, S_{n-1}} \circ \pi_{S_{n-1}}=\pi_{S_{n}}$, respectively, $\pi_{S_{n-1} \text { (sheaf) }}^{*} \circ \pi_{S_{n}, S_{n-1} \text { (sheaf) }}^{*}=\pi_{S_{n} \text { (sheaf) }}^{*}$, the algebra homomorphism

$$
\pi_{S_{n}, S_{n-1} \text { (sheaf) }}^{*}: H_{\text {sheaf }}^{*}\left(W / S_{n} ; K\right) \rightarrow H_{\text {sheaf }}^{*}\left(W / S_{n-1} ; K\right)
$$

is isomorphic to the inclusion $i: H_{\text {sheaf }}^{*}(W ; K)^{S_{n}} \rightarrow H_{\text {sheaf }}^{*}(W ; K)^{S_{n-1}}$. Therefore, we have a group-theoretic transfer $i: A^{S_{n}} \rightarrow A^{S_{n-1}}, \tau: A^{S_{n-1}} \rightarrow A^{S_{n}}$, where $A^{*}$ is the algebra $H_{\text {sheaf }}^{*}(W ; K)$, which is an $n$-transfer in the sense of our definition. By Theorem 2.1.6, we have an estimate on the multiplicative lengths of the algebras $A^{S_{n}}$ and $A^{S_{n-1}}$ : $l\left(A^{S_{n}}\right)+1 \geq \frac{l\left(A^{S_{n-1}}\right)+1}{n}$.

But we also have the isomorphisms

$$
\begin{gathered}
A^{S_{n}}=H_{\text {sheaf }}^{*}(W ; K)^{S_{n}} \cong H_{\text {sheaf }}^{*}\left(W / S_{n} ; K\right) \cong H_{\text {sheaf }}^{*}(Y ; K) \cong H^{*}(Y ; K), \\
A^{S_{n-1}}=H_{\text {sheaf }}^{*}(W ; K)^{S_{n-1}} \cong H_{\text {sheaf }}^{*}\left(W / S_{n-1} ; K\right) \cong H_{\text {sheaf }}^{*}(X ; K) \cong H^{*}(X ; K) .
\end{gathered}
$$

Thus, we have constructed $n$-transfers $f^{*}: H^{*}(Y ; \mathbb{Q}) \rightarrow H^{*}(X ; \mathbb{Q}), \tau: H^{*}(X ; \mathbb{Q}) \rightarrow$ $H^{*}(Y ; \mathbb{Q})$ and $f^{*}: H^{*}\left(Y ; \mathbb{Z}_{p}\right) \rightarrow H^{*}\left(X ; \mathbb{Z}_{p}\right), \tau: H^{*}\left(X ; \mathbb{Z}_{p}\right) \rightarrow H^{*}\left(Y ; \mathbb{Z}_{p}\right), p>n$, which yield, in particular, the estimates on the rational and $\mathbb{Z}_{p}, p>n$, cohomological lengths of $X$ and $Y$ :

$$
l(Y)+1 \geq \frac{l(X)+1}{n}, \quad l_{p}(Y)+1 \geq \frac{l_{p}(X)+1}{n}, \quad p>n .
$$

Now we consider the case $n=2$ and show that the obtained estimates $l(Y)+1 \geq \frac{l(X)+1}{2}$ and $l_{p}(Y)+1 \geq \frac{l_{p}(X)+1}{2}, p>2$, are sharp. It is not difficult to check that any 2fold Smith-Dold branched covering is the quotient map by the action of a continuous involution. Suppose $X=T^{m}$ is an $m$-dimensional torus, $m \geq 1$, and $\sigma: T^{m} \rightarrow T^{m}$, 
$\sigma(x)=-x \forall x \in T^{m}$, is an involution. We have a 2-fold Smith-Dold branched covering $\pi: T^{m} \rightarrow T^{m} / \sigma=Y$.

Suppose $K$ is a field of characteristic different from 2. The Künneth formula implies that $H^{*}\left(T^{m} ; K\right)=\Lambda_{K}\left(u_{1}, \ldots, u_{m}\right)$. Moreover, we can choose a basis $u_{1}, \ldots, u_{m}$ of $H^{1}\left(T^{m} ; K\right)=H_{1}\left(T^{m} ; K\right)^{*}$ dual to the basis $\alpha_{1}, \ldots, \alpha_{m} \in H_{1}\left(T^{m} ; K\right)$, whose elements are realized by the elementary one-cycles: the circles

$$
\left\{(1, \ldots, 1, \underbrace{e^{i \varphi}}_{j \text {-th place }}, 1, \ldots, 1) \mid 0 \leq \varphi \leq 2 \pi\right\} \subset T^{m}=S^{1} \times \ldots \times S^{1}, 1 \leq j \leq m .
$$

The operator $\sigma_{*}: H_{1}\left(T^{m} ; K\right) \rightarrow H_{1}\left(T^{m} ; K\right)$, corresponding to our involution $\sigma$, obviously sends $\alpha_{i}$ to $\sigma_{*}\left(\alpha_{i}\right)=-\alpha_{i}, 1 \leq i \leq m$.

The operator $\sigma^{*}: H^{1}\left(T^{m} ; K\right) \rightarrow H^{1}\left(T^{m} ; K\right)$ is adjoint to $\left.\sigma_{*}\right|_{H_{1}\left(T^{m} ; K\right)}=-E$, and therefore, $\left.\sigma^{*}\right|_{H^{1}\left(T^{m} ; K\right)}=-E$. Thus, the automorphism $\sigma^{*}: \Lambda_{K}\left(u_{1}, \ldots, u_{m}\right) \rightarrow \Lambda_{K}\left(u_{1}\right.$, $\left.\ldots, u_{m}\right)$ acts on the generators $u_{i}, 1 \leq i \leq m$, of the exterior algebra as follows: $\sigma^{*}\left(u_{i}\right)=$ $-u_{i}, 1 \leq i \leq m$. It is clear that $\sigma^{*}(u)=(-1)^{|u|} u$ for any homogeneous element of $\Lambda_{K}\left(u_{1}, \ldots, u_{m}\right)$. Therefore, the invariant subalgebra $\Lambda_{K}\left(u_{1}, \ldots, u_{m}\right)^{\mathbb{Z}_{2}}$ is the subalgebra $\Lambda_{K}^{2 *}\left(u_{1}, \ldots, u_{m}\right)$ of even-degree cohomology.

According to the above theorem on the cohomology of the quotient by an action of a finite group, we have an isomorphism

$$
H^{*}(Y ; K)=H^{*}\left(T^{m} / \sigma ; K\right) \cong H^{*}(X ; K)^{\mathbb{Z}_{2}} \cong \Lambda_{K}^{2 *}\left(u_{1}, \ldots, u_{m}\right) .
$$

It is clear that $l\left(H^{*}(X ; K)\right)=l\left(\Lambda_{K}\left(u_{1}, \ldots, u_{m}\right)\right)=m$. We determine $l\left(H^{*}(Y ; K)\right)=$ $l\left(\Lambda_{K}^{2 *}\left(u_{1}, \ldots, u_{m}\right)\right)$. If $m$ is even, $m=2 s, s \geq 1$, then $l\left(\Lambda_{K}^{2 *}\left(u_{1}, \ldots, u_{2 s}\right)\right)=s$, and in this case, our estimate $l(Y)+1=l_{p}(Y)+1=s+1 \geq \frac{2 s+1}{2}=\frac{l(X)+1}{2}=\frac{l_{p}(X)+1}{2} \forall p>2$, $\forall s \geq 1$ is sharp.

If $m$ is odd, $m=2 s-1, s \geq 1$, then $l\left(\Lambda_{K}^{2 *}\left(u_{1}, \ldots, u_{2 s-1}\right)\right)=s-1$, and we see that, in this case as well, our estimate $l(Y)+1=l_{p}(Y)+1=(s-1)+1 \geq \frac{(2 s-1)+1}{2}=\frac{l(X)+1}{2}=$ $\frac{l_{p}(X)+1}{2} \forall p>2, \forall s \geq 1$, is sharp.

Now suppose that $l(X)=0$ or $l_{p}(X)=0, p>2$, and $f: X \rightarrow Y$ is an arbitrary 2-fold Smith-Dold branched covering. Since we always have $0 \leq l(Y) \leq l(X)$, respectively $0 \leq l_{p}(Y) \leq l_{p}(X)$, we have a sharp estimate $l(Y)+1=0+1 \geq \frac{0+1}{2}=\frac{l(X)+1}{2}$, respectively $l_{p}(Y)+1=0+1 \geq \frac{0+1}{2}=\frac{l_{p}(X)+1}{2}, p>2$, in this trivial case as well.

Thus, we have constructed examples of 2-fold Smith-Dold branched coverings $f: X \rightarrow$ $Y$ of any given lengths $l(X)$ and $l_{p}(X)$, for which our estimates $l(Y)+1 \geq \frac{l(X)+1}{2}$, $l_{p}(Y)+1 \geq \frac{l_{p}(X)+1}{2} \forall p>2$, are sharp.

2.3. The case of manifolds. The results of the previous section imply that the following two well-known in topology classes of maps belong to the class of $n$-fold Smith-Dold branched coverings:

(1) regular finite coverings $f: X \rightarrow Y$;

(2) the quotient map by an action of a finite group $\pi: X \rightarrow X / G$.

In particular, Theorem 2.2.3 applies in both cases, which yields the estimates $l(Y)+1 \geq$ $\frac{l(X)+1}{n}$ and $l_{p}(Y)+1 \geq \frac{l_{p}(X)+1}{n}, p>n$.

In this section, we shall construct another important class of $n$-fold Smith-Dold branched coverings. First, we need to recall the definition of a finite-to-one map.

Definition 2.3.1. Suppose $X, Y$ are topological spaces. A continuous map $f: X \rightarrow Y$ is said to be finite-to-one if for any $y \in Y$ its preimage $f^{-1}(y)$ consists of finitely many points (this number may be unbounded over $y$ ). 
Consider an arbitrary continuous open-closed finite-to-one map $f: M^{m} \rightarrow N^{m}$ between connected topological $m$-dimensional manifolds without boundary. The structure of such maps was described in a well-known paper by A. V. Chernavsky [16] in 1964.

Theorem (A. V. Chernavsky). Suppose $f: M^{m} \rightarrow N^{m}, m \geq 2$, is a map of the above type with branching set

$$
B_{f}=\left\{x \in M^{m} \mid f \text { is not a local homeomorphism at } x\right\} .
$$

Then $f$ is of bounded multiplicity, the points in $N^{m}$ of maximal multiplicity form an everywhere dense open subset, and $\operatorname{dim} B_{f} \leq m-2$.

In 1978, I. Berstein and A. L. Edmonds [2] proved, using this theorem, the following result.

Proposition (I. Berstein and A. L. Edmonds). Suppose $M^{m}$ and $N^{m}$ are connected closed oriented $m$-dimensional manifolds, and $f: M^{m} \rightarrow N^{m}$ a continuous open finiteto-one map of degree $\pm n$. Then there are a locally compact Hausdorff space $X$ with an action of a finite group $G \subset S_{n}$ and a subgroup $H \subset G$ of index $n$ such that $M^{m}=X / H$, $N^{m}=X / G$, and the map $f: M^{m} \rightarrow N^{m}$ coincides with the map $\pi_{G, H}: X / H \rightarrow X / G$.

In fact, in the proof of this result, I. Berstein and A. L. Edmonds never used the fact that $M^{m}$ and $N^{m}$ are closed and oriented (it suffices to require that $f$ be closed), and only used the inequality $\operatorname{dim} B_{f} \leq m-2$ and an equivalent inequality $\operatorname{dim} f\left(B_{f}\right) \leq$ $m-2$. Based on this and on the theorem of A. Dold, we can say that I. Berstein and A. L. Edmonds implicitly proved the following proposition, for which we give here a complete and independent proof.

Proposition 2.3.2. Any continuous open-closed finite-to-one map $f: M^{m} \rightarrow N^{m}, m \geq$ 2 , between connected topological m-dimensional manifolds without boundary is an $n$-fold Smith-Dold branched covering, where $n$ is the maximum multiplicity of $f$.

Proof. We first remark that the map $f: M^{m} \rightarrow N^{m}$ is surjective. Indeed, $f\left(M^{m}\right) \subset$ $N^{m}$ is an open-closed nonempty subset of the connected manifold $N^{m}$ and, therefore, $f\left(M^{m}\right)=N^{m}$. By the theorem of A. V. Chernavsky, the map $f: M^{m} \rightarrow N^{m}$ is of bounded multiplicity. Let $n$ be the maximum multiplicity of $f$.

By ([25, p. 173, Th. 2]), if $f: X \rightarrow Y$ is a continuous open-closed surjective map of topological spaces, then the full preimage map $f^{-1}: Y \rightarrow \exp (X)$ is continuous, where $\exp (X)$ is the set of all nonempty closed subsets of $X$ endowed with the Vietoris topology. We now recall the definition of this topology.

For any subset $A \subset X$ let $\exp (A, X)$ be the subset $\{F \in \exp (X) \mid F \subset A\} \subset \exp (X)$. The Vietoris topology on $\exp (X)$ is the smallest of the topologies for which $\exp (A, X)$ is closed for any closed subset and open for any open subset $A \subset X$. Standard arguments show that the sets $O\left\langle U_{1}, \ldots, U_{n}\right\rangle=\left\{F \in \exp (X) \mid F \subset \bigcup_{i=1}^{n} U_{i}, F \cap U_{i} \neq \varnothing \forall 1 \leq i \leq\right.$ $n\} \subset \exp (X)$, where $n \geq 1$, and $U_{1}, \ldots, U_{n} \subset X$ are open, form a base of the Vietoris topology.

We can apply these facts to our map $f: M^{m} \rightarrow N^{m}$. Fix a point $y \in N^{m}$. Its full preimage $f^{-1}(y)$ consists of $k$ points $f^{-1}(y)=\left\{x_{1}, \ldots, x_{k}\right\}, 1 \leq k \leq n$. Now we shall describe how to assign a natural multiplicity $n_{i}$ to each point $x_{i}, 1 \leq i \leq k$, such that $n_{1}+\ldots+n_{k}=n$, and then show that the corresponding map $g: N^{m} \rightarrow \operatorname{Sym}^{n} M^{m}$, $g(y)=\left[n_{1} x_{1}, \ldots, n_{k} x_{k}\right]$ is continuous.

Consider open disjoint subsets $U_{1} \ni x_{1}, \ldots, U_{k} \ni x_{k}$. Since $f^{-1}(y) \in O\left\langle U_{1}, \ldots, U_{k}\right\rangle$ and the map $f^{-1}: N^{m} \rightarrow \exp \left(M^{m}\right)$ is continuous, there exists a small connected neighborhood $V \ni y$ such that $f^{-1}: V \rightarrow O\left\langle U_{1}, \ldots, U_{k}\right\rangle$. This means that for any $y^{\prime} \in V$, the finite set $f^{-1}\left(y^{\prime}\right)$ lies in $U_{1} \sqcup \ldots \sqcup U_{k}$ and $f^{-1}\left(y^{\prime}\right) \cap U_{1} \neq \varnothing, \ldots, f^{-1}\left(y^{\prime}\right) \cap U_{k} \neq \varnothing$. 
Consider the open set $f^{-1}(V) \subset U_{1} \sqcup \ldots \sqcup U_{k}$. Since $f^{-1}(V)$ is an open subset of the manifold $M^{m}$, it is itself a manifold, consisting of a disjoint union of at most countably many connected components $f^{-1}(V)=\bigsqcup_{i=1}^{N} V_{i}, 1 \leq N \leq \infty$. We now prove an auxiliary result.

Suppose $f: X \rightarrow Y$ is a surjective continuous open-closed map of topological spaces and $B \subset Y$ is an arbitrary subspace. Then the restriction $f: f^{-1}(B)=A \rightarrow B$ is also an open-closed map. Indeed, suppose $U \subset A$ is open in $A$. Then there exists an open subset $U_{1} \subset X$ such that $U=A \cap U_{1}$. This implies that $f(U)=B \cap f\left(U_{1}\right)$ is open in $B$, since $f\left(U_{1}\right)$ is open in $Y$. Similarly, for a closed subset $F \subset A$ there is a subset $F_{1} \subset X$ such that $F=A \cap F_{1}$, and, therefore, $f(F)=B \cap f\left(F_{1}\right)$ is a closed subset of $B$ because $f\left(F_{1}\right)$ is closed in $Y$. This proves the desired assertion.

By the above argument, the map $f: f^{-1}(V)=\bigsqcup_{i=1}^{N} V_{i} \rightarrow V$ is open-closed. Since each connected component $V_{i}, 1 \leq i \leq N$, of $f^{-1}(V)$ is open-closed in $f^{-1}(V)$, the maps $f: V_{i} \rightarrow V, 1 \leq i \leq N$, are also open-closed. Since we chose $V$ to be connected, we have $f\left(V_{i}\right)=V \forall 1 \leq i \leq N$. As $f^{-1}(V) \subset U_{1} \sqcup \ldots \sqcup U_{k}$, all connected components $V_{i}$, $1 \leq i \leq N$, break into groups lying in $U_{1}$, in $U_{2}, \ldots$, in $U_{k}$. We shall show that in each $U_{i}, 1 \leq i \leq k$, there is exactly one connected component $V_{j}, 1 \leq j \leq N$.

For any fixed set $U_{i}, 1 \leq i \leq k$, we can find one component $V_{j}$ containing a point $x_{i} \in U_{i}, x_{i} \in f^{-1}(y)$. Suppose there exists another component $V_{j^{\prime}} \neq V_{j}, V_{j^{\prime}} \cap V_{j}=\varnothing$, $V_{j^{\prime}} \subset U_{i}$. As we saw before, the map $f: V_{j^{\prime}} \rightarrow V$ is surjective. Therefore, there is a point $x_{0} \in V_{j^{\prime}} \subset U_{i}, f\left(x_{0}\right)=y, x_{0} \neq x_{i}$. But then $f^{-1}(y) \cap U_{i}$ contains at least two points: $x_{i}$ and $x_{0}, x_{0} \neq x_{i}$, which cannot happen since $U_{i} \cap f^{-1}(y)=\left\{x_{i}\right\}, 1 \leq i \leq k$. The obtained contradiction proves the claim.

Thus, we have showed that $f^{-1}(V)$ breaks into a union of $k$ connected components $f^{-1}(V)=V_{1} \sqcup \ldots \sqcup V_{k}, x_{i} \in V_{i} \subset U_{i} \forall 1 \leq i \leq k$. As we saw, the map $f: V_{i} \rightarrow V$ $\forall 1 \leq i \leq k$ is surjective and open-closed. It is clear that it is finite-to-one. Let $n_{i}$ be the maximum multiplicity of $f: V_{i} \rightarrow V, 1 \leq i \leq k$. By the theorem of A. V. Chernavsky, the subsets $W_{i} \subset V, 1 \leq i \leq k$, on which the maximum multiplicity $n_{i}$ of $f: V_{i} \rightarrow V$ is attained are everywhere dense in $V$.

Now, for any point $y^{\prime} \in W_{1} \cap \ldots \cap W_{k} \subset V$ its full preimage $f^{-1}\left(y^{\prime}\right) \subset M^{m}$ consists of $n_{1}+\ldots+n_{k}$ points (exactly $n_{i}$ points in each $V_{i}, 1 \leq i \leq k$ ). But the set $W_{1} \cap \ldots \cap W_{k}$ is open and everywhere dense in $V$, and, therefore, it intersects the open everywhere dense set $W_{0} \subset N^{m}$ consisting of the points $y^{\prime}$ whose full preimage $f^{-1}\left(y^{\prime}\right)$ in $M^{m}$ has the maximum number, $n$, of points. Thus, taking any point $y^{\prime} \in W_{0} \cap W_{1} \cap \ldots \cap W_{k}$, we see that $n_{1}+\ldots+n_{k}=n$.

Now we want to show that for any connected neighborhood $V^{\prime}, y \in V^{\prime} \subset V$, its full preimage $f^{-1}\left(V^{\prime}\right)$ breaks into connected components $f^{-1}\left(V^{\prime}\right)=V_{1}^{\prime} \sqcup \ldots \sqcup V_{k}^{\prime}$ such that $x_{i} \in V_{i}^{\prime} \subset V_{i}, 1 \leq i \leq k$. Indeed, for this it suffices to repeat our arguments with substitutions $U_{i} \rightarrow V_{i}, 1 \leq i \leq k, V \rightarrow V^{\prime}$. We can do this, since $f^{-1}: V \rightarrow O\left\langle V_{1}, \ldots, V_{k}\right\rangle$ and, therefore, $f^{-1}: V^{\prime} \rightarrow O\left\langle V_{1}, \ldots, V_{k}\right\rangle$. We have, as before, that $f: V_{i}^{\prime} \rightarrow V^{\prime}, 1 \leq i \leq$ $k$, are surjective finite-to-one open-closed maps. Let $n_{i}^{\prime}, 1 \leq i \leq k$, be their maximum multiplicities. For any point $y^{\prime} \in V^{\prime} \cap W_{1} \cap \ldots \cap W_{k} \neq \varnothing$ its full preimage $f^{-1}\left(y^{\prime}\right)$ in $V_{i}^{\prime}$ coincides with its full preimage in $V_{i}$ and consists of $n_{i}$ points, $1 \leq i \leq k$. Therefore, $n_{i}^{\prime} \geq n_{i}, 1 \leq i \leq k$. But $n_{i}^{\prime} \leq n_{i}, 1 \leq i \leq k$, since the full preimage of any point $y^{\prime} \in V^{\prime} \subset V$ under the map $f: V_{i}^{\prime} \rightarrow V^{\prime}$ coincides with the full preimage of the same point under the map $f: V_{i} \rightarrow V$. It now follows that $n_{i}^{\prime}=n_{i} \forall 1 \leq i \leq k$. Thus, we have proved that for any connected neighborhood $y \in V^{\prime} \subset V$ its full preimage $f^{-1}\left(V^{\prime}\right)$ breaks into connected components $f^{-1}\left(V^{\prime}\right)=V_{1}^{\prime} \sqcup \ldots \sqcup V_{k}^{\prime}, x_{i} \in V_{i}^{\prime} \subset V_{i}, 1 \leq i \leq k$, and that the maximum multiplicity of $f: V_{i}^{\prime} \rightarrow V^{\prime}$ equals $n_{i}, 1 \leq i \leq k$, and does not depend 
on $V^{\prime}$. Moreover, $n_{1}+\ldots+n_{k}=n$. It is that invariant multiplicity $n_{i}$ that we assign to the point $x_{i}$ in the preimage of $y \in N^{m}, 1 \leq i \leq k$.

We remark that when the diameter of the connected neighborhood $V^{\prime} \ni y, V^{\prime} \subset$ $V$, tends to zero (i.e., when $V^{\prime} \ni y$ becomes arbitrarily small) the diameters of all connected components $V_{i}^{\prime}, 1 \leq i \leq k$, of its preimage $f^{-1}\left(V^{\prime}\right)=V_{1}^{\prime} \sqcup \ldots \sqcup V_{k}^{\prime}$ also tend to zero (i.e., the $V_{i}^{\prime}, 1 \leq i \leq k$ become arbitrarily small). Indeed, fix arbitrarily small disjoint neighborhoods $x_{1} \in U_{1}^{\prime} \subset V_{1}, \ldots, x_{k} \in U_{k}^{\prime} \subset V_{k}$. Since $f^{-1}: N^{m} \rightarrow \exp \left(M^{m}\right)$ is continuous, there is a small connected neighborhood $V^{\prime} \ni y, V^{\prime} \subset V$, such that $f^{-1}: V^{\prime} \rightarrow O\left\langle U_{1}^{\prime}, \ldots, U_{k}^{\prime}\right\rangle$. But for $V^{\prime} \ni y, V^{\prime} \subset V$, we already know that its full preimage breaks into connected components $f^{-1}\left(V^{\prime}\right)=V_{1}^{\prime} \sqcup \ldots \sqcup V_{k}^{\prime}$ with $x_{i} \in V_{i}^{\prime}$, $1 \leq i \leq k$. As $f^{-1}: V^{\prime} \rightarrow O\left\langle U_{1}^{\prime}, \ldots, U_{k}^{\prime}\right\rangle$, we have $V_{1}^{\prime} \sqcup \ldots \sqcup V_{k}^{\prime} \subset U_{1}^{\prime} \sqcup \ldots \sqcup U_{k}^{\prime}$. Since $V_{i}^{\prime}, 1 \leq i \leq k$, it follows that $V_{i}^{\prime} \subset U_{i}^{\prime} \forall 1 \leq i \leq k$. Thus, the connected components $x_{1} \in V_{1}^{\prime}, \ldots, x_{k} \in V_{k}^{\prime}$ of the preimage $f^{-1}\left(V^{\prime}\right)$ become arbitrarily small when $V^{\prime} \ni y$ is sufficiently small, which proves the claim.

But then the multiplicity $n_{i}$ assigned to $x_{i}$, which coincides with the maximum multiplicity of $f: V_{i}^{\prime} \rightarrow V^{\prime}$ for all sufficiently small connected neighborhoods $V^{\prime} \ni y$, depends only on the local behavior of $f: M^{m} \rightarrow N^{m}$ at $x_{i}, 1 \leq i \leq k$. Using standard arguments, it is not difficult to show that the so-defined multiplicity $n_{x}$ at an arbitrary point $x \in M^{m}$ coincides with the local degree of $f: M^{m} \rightarrow N^{m}$ at $x$, taken with the positive sign.

Thus, we have constructed a map $g: N^{m} \rightarrow \operatorname{Sym}^{n} M^{m}$ such that $\langle g(y)\rangle=f^{-1}(y)$ $\forall y \in N^{m}$. We want to show that this map is continuous. First, we recall one fact.

Let $X$ be a Hausdorff space, and $\operatorname{Sym}^{n} X=X^{n} / S_{n}$ its $n$-th symmetric power. Fix a point $\left[n_{1} x_{1}, \ldots, n_{k} x_{k}\right] \in \operatorname{Sym}^{n} X, n_{1}+\ldots+n_{k}=n, x_{i} \neq x_{j}, i \neq j$. It is easy to check that the sets

$$
\begin{aligned}
& W_{U_{1}, n_{1} ; \ldots ; U_{k}, n_{k}}=\left\{\left[x_{1}^{\prime}, \ldots, x_{n}^{\prime}\right] \in \operatorname{Sym}^{n} X \mid\right. \\
& \quad \text { exactly } n_{1} \text { points from } x_{i}^{\prime}, 1 \leq i \leq n, \text { are in } U_{1}, \ldots, \\
& \text { exactly } \left.n_{k} \text { points from } x_{i}^{\prime}, 1 \leq i \leq n, \text { are in } U_{k}\right\},
\end{aligned}
$$

where $x_{1} \in U_{1} \subset X, \ldots, x_{k} \in U_{k} \subset X$ are open disjoint sets, form an open base of the topology of $\operatorname{Sym}^{n} X$ at the point $\left[n_{1} x_{1}, \ldots, n_{k} x_{k}\right]$.

Take an arbitrary point $y \in N^{m}$ and its image $g(y)=\left[n_{1} x_{1}, \ldots, n_{k} x_{k}\right] \in \operatorname{Sym}^{n} M^{m}$, $f^{-1}(y)=\left\{x_{1}, \ldots, x_{k}\right\}$. What we mentioned about the topology of a symmetric power implies that to prove the proposition, we need, for any collection of open disjoint sets $x_{1} \in U_{1} \subset M^{m}, \ldots, x_{k} \in U_{k} \subset M^{m}$, to find a small open neighborhood $y \in V \subset N^{m}$ such that $g: V \rightarrow W_{U_{1}, n_{1} ; \ldots ; U_{k}, n_{k}}$.

For $y \in N^{m}$, we already constructed a connected neighborhood $V_{y} \ni y$ such that for any connected neighborhood $y \in V \subset V_{y}$ its full preimage $f^{-1}(V)$ breaks into connected components $f^{-1}(V)=V_{1} \sqcup \ldots \sqcup V_{k}, x_{i} \in V_{i}, 1 \leq i \leq k$, and the restriction $f: V_{i} \rightarrow V$ is a surjective open-closed map of maximum multiplicity $n_{i}, 1 \leq i \leq k$. Therefore, since for any sufficiently small neighborhood $V \ni y$ we have $f^{-1}: V \rightarrow O\left\langle U_{1}, \ldots, U_{k}\right\rangle \subset \exp \left(M^{m}\right)$, we can find a sufficiently small connected open neighborhood $y \in V \subset V_{y}$ such that $f^{-1}(V)=V_{1} \sqcup \ldots \sqcup V_{k} \subset U_{1} \sqcup \ldots \sqcup U_{k}, x_{i} \in V_{i} \subset U_{i}, 1 \leq i \leq k$.

Consider an arbitrary point $y^{\prime} \in V$. Its full preimage $f^{-1}\left(y^{\prime}\right)$ breaks into a disjoint union

$$
f^{-1}\left(y^{\prime}\right)=\left\{x_{1,1}^{\prime}, \ldots, x_{1, l_{1}}^{\prime}\right\} \sqcup \ldots \sqcup\left\{x_{k, 1}^{\prime}, \ldots, x_{k, l_{k}}^{\prime}\right\},
$$

where $\left\{x_{i, 1}^{\prime}, \ldots, x_{i, l_{i}}^{\prime}\right\} \subset V_{i} \subset U_{i}$ is the full preimage of $y^{\prime}$ under the map $f: V_{i} \rightarrow V$, $1 \leq i \leq k$.

As we have already shown, the multiplicity $n_{i, j}$ assigned to $x_{i, j}, 1 \leq i \leq k, 1 \leq j \leq l_{i}$, is defined locally. Therefore, for a fixed $i, 1 \leq i \leq k$, the multiplicities $n_{i, 1}, \ldots, n_{i, l_{i}}$ 
corresponding to the points $x_{i, 1}, \ldots, x_{i, l_{i}} \in V_{i}$ can be defined via the map $f: V_{i} \rightarrow V$. But we have already proved that the total multiplicity over all points of the preimage of any $y \in Y^{m}$ under any map of connected $m$-dimensional manifolds without boundary $f: X^{m} \rightarrow Y^{m}$ of our type equals the maximum multiplicity of $f$. Therefore, in our situation, we have $n_{i, 1}+\ldots+n_{i, l_{i}}=n_{i} \forall 1 \leq i \leq k$, which implies the desired inclusion

$$
\begin{aligned}
g\left(y^{\prime}\right)=\left[n_{1,1} x_{1,1}^{\prime}, \ldots, n_{1, l_{1}} x_{1, l_{1}}^{\prime}, \ldots, n_{k, 1} x_{k, 1}^{\prime}, \ldots, n_{k, l_{k}} x_{k, l_{k}}^{\prime}\right] & \\
\in W_{U_{1}, n_{1} ; \ldots ; U_{k}, n_{k}} \forall y^{\prime} & \in V .
\end{aligned}
$$

I. Berstein and A. L. Edmonds [2] were interested, in particular, in the following problem. Suppose $M^{m}$ and $N^{m}$ are closed connected oriented $m$-dimensional manifolds and $f: M^{m} \rightarrow N^{m}$ a continuous open finite-to-one map of degree $\pm n$ (it is clear that $n$ equals the maximum multiplicity of $f$ ). It makes sense to call such maps $n$-fold branched coverings. How can we estimate, in terms of the rational cohomology $H^{*}\left(M^{m} ; \mathbb{Q}\right)$ and $H^{*}\left(N^{m} ; \mathbb{Q}\right)$, the number $n$ ? This problem goes back to Alexander's well-known paper [1], in which he proved the following theorem.

Theorem (Alexander). For any closed oriented $m$-dimensional $\mathrm{PL}$ manifold $M^{m}$ there exists an open piecewise linear finite-to-one map from $M^{m}$ to the sphere $S^{m}$.

I. Berstein and A. L. Edmonds, using their proposition (mentioned above) and their own algebraic technique, proved the following theorem. (As usual, $l(X)$ denotes the rational cohomological length of $X$.)

Theorem (I. Berstein and A. L. Edmonds). Suppose $M^{m}$ and $N^{m}$ are connected closed oriented $m$-dimensional manifolds and $f: M^{m} \rightarrow N^{m}$ a continuous open finite-to-one map of degree $\pm n$. Then $l\left(N^{m}\right) \geq \frac{l\left(M^{m}\right)}{n}$.

The proof of this estimate relies on the rational Poincaré duality and, because of that, only applies to the oriented case. It is easy to give an example when this estimate fails if at least $N^{m}$ is nonorientable. Consider a two-fold covering $f: S^{2 m} \rightarrow \mathbb{R} P^{2 m}$. The space $\mathbb{R} P^{2 m}$ has rational cohomology only in dimension zero; hence $l\left(\mathbb{R} P^{2 m}\right)=0$.

In this case, the Berstein-Edmonds estimate becomes $0=l\left(\mathbb{R} P^{2 m}\right) \geq \frac{l\left(S^{2 m}\right)}{2}=\frac{1}{2}$, and is, therefore, false. Note that our general estimate from Theorem 2.2.3, $l\left(N^{m}\right)+1$ $\geq \frac{l\left(M^{m}\right)+1}{n}$, is a little weaker than the Berstein-Edmonds estimate, but it works also in the case of nonorientable $M^{m}$ and $N^{m}$. In the just considered example, we have $1=l\left(\mathbb{R P}^{2 m}\right)+1 \geq \frac{l\left(S^{2 m}\right)+1}{2}=1$. We also remark that using the rational Poincaré duality and our technique of $n$-transfer, we can deduce the Berstein-Edmonds estimate. In fact, I. Berstein and A. L. Edmonds obtained an estimate from below on the degree $n$ of a branched covering $f: M^{m} \rightarrow N^{m}$ for fixed oriented $m$-dimensional manifolds $M^{m}$ and $N^{m}$. In particular, this allowed us to deduce that for the torus $T^{m}$, the minimum degree $n$ of its branched covering over the sphere $S^{m}$ (which exists by the theorem of Alexander) would be at least $m$.

We remark that the usual branched coverings in the theory of smooth manifolds (with branching along codimension-two submanifolds) are a particular case of the considered general situation. As for holomorphic maps $f: M^{m} \rightarrow N^{m}$ between complex manifolds of the same dimension, the following result is known and can be found, for example, in [26. p. 132] in a stronger form.

Proposition. A holomorphic map $f: M^{m} \rightarrow N^{m}$ between complex manifolds of the same dimension is open if and only if it is discrete, i.e., $f^{-1}(y)$ is a discrete subset of $M^{m}$ for any $y \in N^{m}$.

The following result now follows immediately from this and Proposition 2.3.2. 
Proposition 2.3.3. Suppose $f: M^{m} \rightarrow N^{m}$ is a closed holomorphic finite-to-one map between connected complex manifolds of the same dimension. Then $f$ is an $n$-fold SmithDold branched covering, where $n$ is the maximum multiplicity of $f$.

\section{Applications to $n$-VAlued topological groups}

3.1. $n$-valued topological groups and $n$-Hopf algebras. The first example of a 2 -valued topological group (2-valued algebraic group on $\mathbb{C} P^{1}$ ) was given by V. M. Buchstaber [6] in 1990. Right after that, he introduced a notion of an $n$-valued topological group. In the particular case of a discrete topology, this yields a notion of an (abstract) $n$-valued group. In this section, we shall consider $n$-valued multiplications defined on path-connected spaces and satisfying some, but not necessarily all, axioms of an $n$-valued topological group. We begin with the main definition.

Definition 3.1.1. Suppose $X$ is a path-connected Hausdorff space. An $n$-valued multiplication is just a continuous map $\mu: X \times X \rightarrow \operatorname{Sym}^{n} X$. We set $\mu(x, y)=x * y$ $\forall x, y \in X$.

(The unit axiom) We say that an $n$-valued multiplication $\mu: X \times X \rightarrow \operatorname{Sym}^{n} X$ satisfies the unit axiom if there exists an element $e \in X$ such that $e * x=x * e=[n x] \forall x \in X$.

(Associativity) An $n$-valued multiplication $\mu: X \times X \rightarrow \operatorname{Sym}^{n} X$ is said to be associative if $(x * y) * z=x *(y * z) \in \operatorname{Sym}^{n^{2}} X \forall x, y, z \in X$.

(Inversion) An $n$-valued multiplication $\mu: X \times X \rightarrow \operatorname{Sym}^{n} X$ is said to have an inverse if there exists a continuous map inv: $X \rightarrow X$ such that $\operatorname{inv}(x) * x \ni e$ and $x * \operatorname{inv}(x) \ni e$ $\forall x \in X$.

If the three axioms above are satisfied, then the quadruple $(X, \mu, e$, inv $)$ is called a structure of an $n$-valued topological group on $X$.

One of the main examples of an $n$-valued topological group is the following construction of a coset $n$-valued group.

Definition 3.1.2. Suppose $G$ is a Lie group and $A$ a finite group (of $n$ elements) of continuous automorphisms of $G$. Let $X$ be the quotient space $G / A$ and $\pi: G \rightarrow X=$ $G / A$ the canonical projection. Define an $n$-valued multiplication $\mu: X \times X \rightarrow \operatorname{Sym}^{n} X$ by setting

$$
\mu(x, y)=\left[\pi\left(\mu_{G}\left(u, a_{i}(v)\right)\right), 1 \leq i \leq n, a_{i} \in A\right] \in \operatorname{Sym}^{n} X \quad \forall x, y \in X,
$$

where $u \in \pi^{-1}(x), v \in \pi^{-1}(y), A=\left\{a_{1}, \ldots, a_{n}\right\}$, and $\mu_{G}: G \times G \rightarrow G$ is the multiplication on $G$. The element $e_{X}=\pi\left(e_{G}\right)$ is then the unit of $X$, and the inversion $\operatorname{inv}_{X}: X \rightarrow X$ is defined by setting $\operatorname{inv}_{X}(x)=\pi\left(u^{-1}\right), u \in \pi^{-1}(x) \forall x \in X$. A direct calculation shows that $\mu$ defines a structure of an $n$-valued group on $X$ with unit $e_{X}$ and inverse $\operatorname{inv}_{X}$. This $n$-valued group $X$ is called a coset $n$-valued group.

An important example of a coset group is the $n$ !-valued coset group $\operatorname{Sym}^{n} G=G \times$ $\ldots \times G / S_{n}$, where $G$ is an arbitrary Lie group. Another example is the 2-valued coset group $T^{k} / \sigma, k \geq 1$, where the involution $\sigma: T^{k} \rightarrow T^{k}$ acts by the rule $\sigma(x)=-x$ $\forall x \in T^{k}$.

Especially important is not the case of real tori, but of abelian varieties, and, in particular, the Jacobians of algebraic curves of genus $g$. When $g=1$, we have the structure of an algebraic 2-valued group on $E_{\tau} /(x \mapsto-x)=\mathbb{C} P^{1}$, discovered by V. M. Buchstaber in 1990. When $g=2$, we have a structure of an algebraic 2-valued group on Kummer surfaces.

It is easy to show that the product $X \times Y$ of an $n$-valued topological group $X$ and an $m$-valued topological group $Y$, endowed with the multiplication

$$
\mu_{X \times Y}\left(\left(x_{1}, y_{1}\right),\left(x_{2}, y_{2}\right)\right)=\left[\left(\mu_{X}\left(x_{1}, x_{2}\right)_{i}, \mu_{Y}\left(y_{1}, y_{2}\right)_{j}\right), 1 \leq i \leq n, 1 \leq j \leq m\right],
$$


is an $n m$-valued topological group with unit $\left(e_{X}, e_{Y}\right)$ and inversion $\operatorname{inv}_{X \times Y}=\operatorname{inv}_{X} \times$ $\operatorname{inv}_{Y}$. It is also easy to see that any $n$-valued topological group $X$ can be viewed as an $n m$-valued group, if each of the $n$ points of the image of the product of two elements is assigned multiplicity $m$.

In this section, we shall also consider a natural homotopical generalization of the notion of an $n$-valued topological group. Examples of a homotopical generalization of a topological group are the famous $H$-groups (in particular, loop spaces). In the case of an $n$-valued multiplication, we shall introduce $n H$-groups, $n H$-monoids, and $n H$-spaces. The main tool for studying $n$-valued topological groups ( $n H$-groups) in this paper is the notion of a graded $n$-Hopf algebra for connected graded commutative algebras $A^{*}$ over a field of characteristic zero. In the case of even-graded (commutative) algebras $A^{2 *}$ this notion was introduced by V. M. Buchstaber and E. G. Rees in 1996. In [13], they proved that for any $n$-valued topological group $X$ its even rational cohomology algebra $H^{\text {even }}(X ; \mathbb{Q})$ carries a structure of a graded $n$-Hopf algebra whenever the odd rational cohomology groups $H^{\text {odd }}(X ; \mathbb{Q})$ vanish. This implies, for example, that there is no structure of a 2 -valued group on $\mathbb{C} P^{m}$ when $m \geq 2$.

We now give a definition of a graded $n$-Hopf algebra (as well as of related notions) in the general case.

Definition 3.1.3. Suppose $K$ is a field of characteristic zero and $A^{*}$ a connected commutative graded $K$-algebra. An algebra $A^{*}$ is called a graded $n$-Hopf prealgebra if there exists a degree zero $K$-linear map $\Delta: A^{*} \rightarrow A^{*} \otimes A^{*}$, called a diagonal such that:

(1) The map $\Delta$ is a $K$-algebra $n$-homomorphism.

(2) $\forall \gamma \in A^{*},|\gamma| \geq 1$, we have $\Delta(\gamma)=n \gamma \otimes 1+1 \otimes n \gamma+\sum_{i=1}^{N} a_{i} \otimes b_{i},\left|a_{i}\right|,\left|b_{i}\right| \geq 1$.

If, in addition, the diagonal $\Delta$ is coassociative, then $A^{*}$ is called a graded $n$-bialgebra.

A graded $n$-bialgebra $A^{*}$ is called a graded $n$-Hopf algebra if there exists an algebra homomorphism $\chi: A^{*} \rightarrow A^{*}$ and $(n-1)$-homomorphisms $\chi_{L}^{\frac{1}{L}}, \chi_{R}^{\frac{1}{R}}: A^{*} \rightarrow A^{*}$ such that the composition $m \circ(\chi \otimes \mathrm{id}) \circ \Delta: A^{*} \rightarrow A^{*}$ coincides with the map $\eta \varepsilon+\chi_{L}^{\frac{1}{L}}$, and the composition $m \circ(\mathrm{id} \otimes \chi) \circ \Delta: A^{*} \rightarrow A^{*}$ coincides with the map $\eta \varepsilon+\chi_{R}^{\perp}$, where $\varepsilon: A^{*} \rightarrow K=A^{0}$ is the augmentation, and $\eta: K \rightarrow A^{*}$ is the algebra homomorphism defining the unit of $A^{*}$.

Lemma 3.1.4. Suppose $X$ is a locally contractible Hausdorff space. Then $\operatorname{Sym}^{n} X$ is also locally contractible for any $n \geq 1$.

Proof. Let $\left[n_{1} x_{1}, \ldots, n_{k} x_{k}\right]$ be an arbitrary point of $\operatorname{Sym}^{n} X, n_{1}+\ldots+n_{k}=n, x_{i} \neq x_{j}$. We need to show that for an arbitrarily small neighborhood $\left[n_{1} x_{1}, \ldots, n_{k} x_{k}\right] \in U \subset$ $\operatorname{Sym}^{n} X$ there is a smaller neighborhood $\left[n_{1} x_{1}, \ldots, n_{k} x_{k}\right] \in V \subset U$, which contracts in $U$ to a point. It is clear that $U$ and $V$ can be chosen from the standard neighborhood basis

$$
\begin{array}{r}
W_{n_{1}, U_{1} ; \ldots ; n_{k}, U_{k}}=\left\{\left[x_{1}^{\prime}, \ldots, x_{n}^{\prime}\right] \in \operatorname{Sym}^{n} X \mid \text { exactly } n_{1} \text { points from } x_{i}^{\prime} \text { lie in } U_{1}, \ldots,\right. \\
\text { exactly } \left.n_{k} \text { points from } x_{i}^{\prime} \text { lie in } U_{k}\right\},
\end{array}
$$

where $x_{1} \in U_{1}, \ldots, x_{k} \in U_{k}$ are disjoint open sets.

Thus, we fix arbitrary disjoint open sets $x_{1} \in U_{1}, \ldots, x_{k} \in U_{k}$ and the corresponding neighborhood $W_{n_{1}, U_{1} ; \ldots ; n_{k}, U_{k}}$. Since $X$ is locally contractible, there are small subneighborhoods $x_{1} \in V_{1} \subset U_{1}, \ldots, x_{k} \in V_{k} \subset U_{k}$ such that $V_{i}$ contracts in $U_{i}, 1 \leq i \leq k$, to a point. Simultaneously applying these contractions to the $n_{i}$ points from $V_{i}, 1 \leq i \leq k$, we have a contraction of $W_{n_{1}, V_{1} ; \ldots ; n_{k}, V_{k}}$ in $W_{n_{1}, U_{1} ; \ldots ; n_{k}, U_{k}}$.

For any commutative graded algebra $A^{*}$ over the field $K$, let $S^{n} A^{*}$ be the subalgebra of all $S_{n}$-invariants in $A^{\otimes n}$, i.e., $S^{n} A^{*}=\left(A^{\otimes n}\right)^{S_{n}}$. There is a natural $n$-homomorphism 


$$
\begin{aligned}
& \chi_{n}\left(A^{*}\right): A^{*} \rightarrow S^{n} A^{*}, \\
& \quad \chi_{n}\left(A^{*}\right)(a)=a \otimes 1 \otimes \ldots \otimes 1+1 \otimes a \otimes \ldots \otimes 1+\ldots+1 \otimes 1 \otimes \ldots \otimes a \quad \forall a \in A^{*} .
\end{aligned}
$$

We want to verify that $\chi_{n}\left(A^{*}\right)$ is indeed an $n$-homomorphism. As the map $\chi_{n}\left(A^{*}\right)$ is obviously linear, we have that $\chi_{n}\left(A^{*}\right)(a b)=(-1)^{|a||b|} \chi_{n}\left(A^{*}\right)(b a) \forall a, b \in A^{*}$. Let $f_{i}: A^{*} \rightarrow A^{\otimes n}, 1 \leq i \leq n$, be the embedding homomorphism into the $i$-th component, $f_{i}(a)=1 \otimes \ldots \otimes \underbrace{a}_{i \text { th place }} \otimes \ldots \otimes 1 \forall a \in A^{*}$. We now have a decomposition $j \circ \chi_{n}\left(A^{*}\right)=f_{1}+\ldots+$ $f_{n}: A^{*} \rightarrow A^{\otimes n}$, where $j: S^{n} A^{*} \rightarrow A^{\otimes n}$ is the canonical inclusion. Therefore, $j \circ \chi_{n}\left(A^{*}\right)$ is an $n$-homomorphism. By Proposition 1.2.4, we have that $\chi_{n}\left(A^{*}\right): A^{*} \rightarrow S^{n} A^{*}$ is an $n$-homomorphism.

The following theorem (as well as its homotopical analog) is the main result of this section.

Theorem 3.1.5. Suppose $X$ is a connected locally contractible paracompact space such that $X^{n^{2}}$ is also paracompact, and $\operatorname{dim} H^{q}(X ; \mathbb{Q})<\infty \forall q \geq 0$. If $X$ carries an $n$-valued multiplication with unit, then its rational singular cohomology algebra $H^{*}(X ; \mathbb{Q})$ carries a structure of a graded $n$-Hopf prealgebra. If the multiplication on $X$ is associative (and has an inverse), then the diagonal defining the structure of the graded $n$-Hopf prealgebra on $H^{*}(X ; \mathbb{Q})$ is coassociative (and has an inverse). In particular, if $X$ is an $n$-valued topological group, then $H^{*}(X ; \mathbb{Q})$ is a graded $n$-Hopf algebra.

Proof. A direct calculation shows that if a finite group $G$ acts on a paracompact space $Z$, then the quotient space $Z / G$ is also paracompact. This also follows from the observation that the canonical projection $\pi: Z \rightarrow Z / G$ is a closed map ([3, Ch. 1, Th. 3.1]), and from the well-known theorem of E. Michael ([21, p. 459, Th. 5.1.33]) asserting that the image of a paracompact space under a closed map is paracompact. It now follows from this fact, Lemma 3.1.4, and Theorem B of Section 2.2, that the singular cohomology of $\operatorname{Sym}^{n} X$ and $X^{n}$ can be canonically identified with their cohomology with coefficients in a constant sheaf. Here we restrict our attention to the constant sheaf of the field $\mathbb{Q}$.

Since $\operatorname{Sym}^{n} X$ is the quotient space of $X^{n}$ by the action of $S_{n}$, we have, by Theorem A of section 2.2 , the following isomorphism $\pi_{n}^{*}: H_{\text {sheaf }}^{*}\left(\operatorname{Sym}^{n} X ; \mathbb{Q}\right) \rightarrow H_{\text {sheaf }}^{*}\left(X^{n} ; \mathbb{Q}\right)^{S_{n}}$, where $\pi_{n}: X^{n} \rightarrow \operatorname{Sym}^{n} X$ is the quotient map. Since singular cohomology in this case can be identified with sheaf cohomology, we have an isomorphism $\pi_{n}^{*}: H^{*}\left(\operatorname{Sym}^{n} X ; \mathbb{Q}\right) \rightarrow$ $H^{*}\left(X^{n} ; \mathbb{Q}\right)^{S_{n}}$. By the Künneth formula, $H^{*}\left(X^{n} ; \mathbb{Q}\right)=H^{*}(X ; \mathbb{Q})^{\otimes n}$, whence an isomorphism $\pi_{n}^{*}: H^{*}\left(\operatorname{Sym}^{n} X ; \mathbb{Q}\right) \rightarrow S^{n} H^{*}(X ; \mathbb{Q})$. Similarly, we have an isomorphism $\pi_{n^{2}}^{*}: H^{*}\left(\operatorname{Sym}^{n^{2}} X ; \mathbb{Q}\right) \rightarrow S^{n^{2}} H^{*}(X ; \mathbb{Q})$.

Suppose now that $X$ admits an $n$-valued multiplication $\mu: X \times X \rightarrow \operatorname{Sym}^{n} X$ with unit $e \in X, \mu(e, x)=\mu(x, e)=[n x] \forall x \in X$. Then $\mu$ induces an algebra homomorphism

$$
\mu^{*}: H^{*}\left(\operatorname{Sym}^{n} X ; \mathbb{Q}\right)=S^{n} H^{*}(X ; \mathbb{Q}) \rightarrow H^{*}(X \times X ; \mathbb{Q})=H^{*}(X ; \mathbb{Q}) \otimes H^{*}(X ; \mathbb{Q}),
$$

which in turn gives rise to an $n$-homomorphism $\Delta:=\mu^{*} \circ \chi_{n}: H^{*}(X ; \mathbb{Q}) \rightarrow H^{*}(X ; \mathbb{Q}) \otimes$ $H^{*}(X ; \mathbb{Q})$, where $\chi_{n}: H^{*}(X ; \mathbb{Q}) \rightarrow S^{n} H^{*}(X ; \mathbb{Q})$ is a natural $n$-algebra homomorphism (for the definition of $\chi_{n}\left(A^{*}\right): A^{*} \rightarrow S^{n} A^{*}$, see p. 128). We claim that the map $\Delta$ : $H^{*}(X ; \mathbb{Q}) \rightarrow H^{*}(X ; \mathbb{Q}) \otimes H^{*}(X ; \mathbb{Q})$ is the desired diagonal, making $H^{*}(X ; \mathbb{Q})$ an $n$ Hopf prealgebra. The fact that the diagonal $\Delta$ is an $n$-homomorphism follows from the definition of this map. It remains to verify the counit axiom.

Suppose $\gamma \in H^{q}(X ; \mathbb{Q}), q \geq 1$. Then

$$
\begin{aligned}
\Delta(\gamma)=\mu^{*}(\gamma \otimes \ldots \otimes 1+\ldots+1 \otimes \ldots \otimes \gamma) & =\alpha \otimes 1+1 \otimes \beta+\sum_{i=1}^{N} \delta_{i}^{\prime} \otimes \delta_{i}^{\prime \prime} \\
& \in H^{*}(X ; \mathbb{Q}) \otimes H^{*}(X ; \mathbb{Q}), \quad\left|\delta_{i}^{\prime}\right|,\left|\delta_{i}^{\prime \prime}\right| \geq 1,1 \leq i \leq N .
\end{aligned}
$$


We shall show that $\alpha=n \gamma$ and $\beta=n \gamma$. We have the following diagram:

$$
X \stackrel{i}{\rightarrow} X \times X \stackrel{\mu}{\longrightarrow} \operatorname{Sym}^{n} X \leftarrow X^{n},
$$

where $i: X \hookrightarrow X \times X, i(x)=(x, e) \forall x \in X$.

By the unit axiom for $\mu$, we have $\mu \circ i=\bar{F}_{n}$, where $\bar{F}_{n}: X \rightarrow \operatorname{Sym}^{n} X, \bar{F}_{n}(x)=[n x]$ $\forall x \in X$, is the diagonal. It is not difficult to check that $i^{*}(\alpha \otimes 1)=\alpha, i^{*}(1 \otimes \beta)=0$ and $i^{*}\left(\delta^{\prime} \otimes \delta^{\prime \prime}\right)=0$ for any $\alpha, \beta, \delta^{\prime}, \delta^{\prime \prime} \in H^{*} \geq 1(X ; \mathbb{Q})$. Therefore, $\alpha=i^{*}\left(\alpha \otimes 1+1 \otimes \beta+\sum_{i=1}^{N} \delta_{i}^{\prime} \otimes\right.$ $\left.\delta_{i}^{\prime \prime}\right)$. On the other hand, we have $\alpha=i^{*} \circ \Delta(\gamma)=i^{*} \circ \mu^{*}(\gamma \otimes \ldots \otimes 1+\ldots+1 \otimes \ldots \otimes \gamma)=$ $\bar{F}_{n}^{*}(\gamma \otimes \ldots \otimes 1+\ldots+1 \otimes \ldots \otimes \gamma)$.

Let $F_{n}: X \rightarrow X^{n}, F_{n}(x)=(x, \ldots, x)$, be the standard diagonal. Since $\bar{F}_{n}=\pi_{n} \circ F_{n}$, we have $\alpha=\bar{F}_{n}^{*}(\gamma \otimes \ldots \otimes 1+\ldots+1 \otimes \ldots \otimes \gamma)=F_{n}^{*}(\gamma \otimes \ldots \otimes 1+\ldots+1 \otimes \ldots \otimes \gamma)$. But $F_{n}^{*}\left(\gamma_{1} \otimes \gamma_{2} \otimes \ldots \otimes \gamma_{n}\right)=\gamma_{1} \gamma_{2} \ldots \gamma_{n} \forall \gamma_{1}, \gamma_{2}, \ldots, \gamma_{n} \in H^{*}(X ; \mathbb{Q})$. It now follows that $\alpha=F_{n}^{*}(\gamma \otimes \ldots \otimes 1+\ldots+1 \otimes \ldots \otimes \gamma)=n \gamma$. Replacing, in diagram (1), the first arrow $i: X \rightarrow X \times X$ by the map $j: X \rightarrow X \times X, j(x)=(e, x) \forall x \in X$, and using the same type of argument, it is not difficult to show that $\beta=n \gamma$. The counit axiom is thus verified. Thus, for any $n$-valued multiplication $\mu: X \times X \rightarrow \operatorname{Sym}^{n} X$ with unit, we have constructed a diagonal $\Delta: H^{*}(X ; \mathbb{Q}) \rightarrow H^{*}(X ; \mathbb{Q}) \otimes H^{*}(X ; \mathbb{Q})$ making $H^{*}(X ; \mathbb{Q})$ a graded $n$-Hopf prealgebra.

We shall now show that if $\mu$ is associative (and has an inverse), then the constructed diagonal $\Delta$ is coassociative (and has an inverse). Set $x * y=\mu(x, y) \in \operatorname{Sym}^{n} X \forall x, y \in X$.

Coassociativity. Suppose the associativity axiom is satisfied for $\mu$, i.e., $(x * y) * z=$ $x *(y * z) \in \operatorname{Sym}^{n^{2}} X \forall x, y, z \in X$. From a formal point of view, this means the equality between the following two compositions:

$$
\begin{aligned}
\operatorname{Sym}^{n^{2}} X \stackrel{\pi_{(2)}}{\longleftarrow} \operatorname{Sym}^{n}\left(\operatorname{Sym}^{n} X\right) \stackrel{\operatorname{Sym}^{n} \mu}{\longleftarrow} \operatorname{Sym}^{n}(X \times X) \\
\stackrel{\pi_{(1 L)}}{\longleftarrow} \operatorname{Sym}^{n} X \times X \stackrel{\mu \times \text { id }}{\longleftarrow} X \times X \times X,
\end{aligned}
$$

(3) $\operatorname{Sym}^{n^{2}} X \stackrel{\pi_{(2)}}{\longleftarrow} \operatorname{Sym}^{n}\left(\operatorname{Sym}^{n} X\right) \stackrel{\operatorname{Sym}^{n} \mu}{\longleftarrow} \operatorname{Sym}^{n}(X \times X)$

$$
\stackrel{\pi_{(1 R)}}{\longleftarrow} X \times \operatorname{Sym}^{n} X \stackrel{\text { id } \times \mu}{\longleftarrow} X \times X \times X .
$$

At the beginning of the proof, we in fact established an isomorphism between the singular cohomology algebras

$$
\pi_{N}^{*}: H^{*}\left(\operatorname{Sym}^{N} Y ; \mathbb{Q}\right) \rightarrow S^{N} H^{*}(Y ; \mathbb{Q})=\left(H^{*}(Y ; \mathbb{Q})^{\otimes N}\right)^{S_{N}}, \quad N \geq 2,
$$

under the following assumptions: (a1) $Y$ is a locally contractible paracompact space; (a2) $Y^{N}$ is a paracompact space; (a3) $\operatorname{dim} H^{q}(Y ; \mathbb{Q})<\infty \forall q \geq 0$.

To apply this isomorphism to diagrams (2) and (3), we need to verify conditions (a1)(a3) for the two new cases: $Y=\operatorname{Sym}^{n} X, N=n$, and $Y=X \times X, N=n$. It is clear that in both cases, (a1) and (a3) hold. It remains to check (a2). In the former case, we need to show that the space $\left(\operatorname{Sym}^{n} X\right)^{n}$ is paracompact. This follows from the fact that $\left(\operatorname{Sym}^{n} X\right)^{n}$ is the quotient

$$
(\underbrace{X^{n} \times \ldots \times X^{n}}_{n \text { times }}) / \underbrace{S_{n} \times \ldots \times S_{n}}_{n \text { times }}
$$

of the paracompact space $X^{n^{2}}$ by the action of the finite group $\left(S_{n}\right)^{n}$. In the latter case, $(X \times X)^{n}=X^{2 n}$ is paracompact as a closed subset of the paracompact space $X^{n^{2}}$. Thus, we can use the structure of the rational singular cohomology of the symmetric powers for all spaces from diagrams (2) and (3). 
Let $A^{*}$ denote the algebra $H^{*}(X ; \mathbb{Q})$. Passing to rational cohomology algebras in diagram (2), we have the following diagram:

$$
\begin{aligned}
S^{n^{2}} A^{*} \stackrel{\pi_{(2)}^{*}=f_{1}}{\longrightarrow} S^{n}\left(S^{n} A^{*}\right) \stackrel{\left(\mathrm{Sym}^{n} \mu\right)^{*}=f_{2}}{\longrightarrow} S^{n}\left(A^{*} \otimes A^{*}\right) \\
\stackrel{\pi_{(1 L)}^{*}=f_{3}}{\longrightarrow} S^{n} A^{*} \otimes A^{*} \stackrel{\mu^{*} \otimes \mathrm{id}=f_{4}}{\longrightarrow} A^{*} \otimes A^{*} \otimes A^{*} .
\end{aligned}
$$

Similarly to diagram (3), we have another diagram in cohomology:

$$
\begin{aligned}
& S^{n^{2}} A^{*} \stackrel{\pi_{(2)}^{*}=g_{1}}{\longrightarrow} S^{n}\left(S^{n} A^{*}\right) \stackrel{\left(\mathrm{Sym}^{n} \mu\right)^{*}=g_{2}}{\longrightarrow} S^{n}\left(A^{*} \otimes A^{*}\right) \\
& \stackrel{\pi_{(1 R)}^{*}=g_{3}}{\longrightarrow} A^{*} \otimes S^{n} A^{*} \stackrel{\mathrm{id} \otimes \mu^{*}=g_{4}}{\longrightarrow} A^{*} \otimes A^{*} \otimes A^{*} .
\end{aligned}
$$

We want to examine diagram $\left(2^{\prime}\right)$.

The maps in that diagram are numbered from right to left by the symbols $f_{1}-f_{4}$. For any natural number $m$, let $\chi_{m}: A^{*} \rightarrow S^{m} A^{*}$ be the natural $m$-homomorphism $\chi_{m}(\gamma)=\gamma \otimes \ldots \otimes 1+\ldots+1 \otimes \ldots \otimes \gamma \forall \gamma \in A^{*}$ of the algebra $A^{*}$. We shall now explicitly determine, one by one, all maps $f_{1}, \ldots, f_{4}$ and compute the image of $\chi_{n^{2}}(\gamma) \in S^{n^{2}} A^{*}$ $\forall \gamma \in A^{q}, \forall q \geq 1$, under the composition $f_{4} \circ f_{3} \circ f_{2} \circ f_{1}$. Fix an arbitrary element $\gamma \in A^{q}$, $q \geq 1$.

Step $f_{1}$. The map $f_{1}: S^{n^{2}} A^{*} \rightarrow S^{n}\left(S^{n} A^{*}\right)$ is induced by the canonical projection $\pi_{(2)}: \operatorname{Sym}^{n}\left(\operatorname{Sym}^{n} X\right) \rightarrow \operatorname{Sym}^{n^{2}} X$. The map $\pi_{(2)}$ can be defined by the following commutative diagram:

$\left(f_{1.1}\right)$

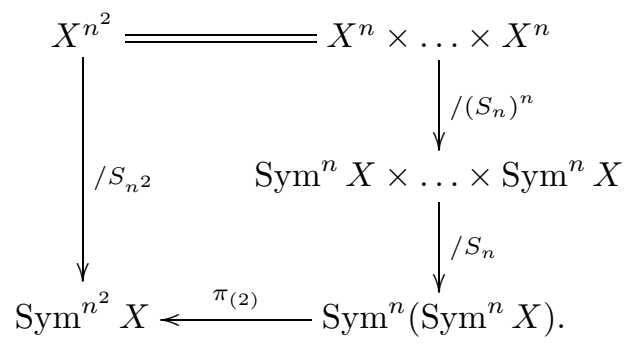

Passing to cohomology, we have a commutative diagram:

$$
\begin{gathered}
A^{\otimes n^{2}}=A^{\otimes n} \otimes \ldots \otimes A^{\otimes n} \\
\cup \\
\cup \quad S^{n} A^{*} \otimes \ldots \otimes S^{n} A^{*} \\
\cup \\
S^{n^{2}} A^{*} \stackrel{f_{1}}{\longrightarrow} S^{n}\left(S^{n} A^{*}\right) .
\end{gathered}
$$

Since all its vertical arrows are identity inclusions, we have that $f_{1}: S^{n^{2}} A^{*} \rightarrow S^{n}\left(S^{n} A^{*}\right)$ is also the identity inclusion. It is not difficult to determine the element $f_{1}\left(\chi_{n^{2}}(\gamma)\right) \in$ $S^{n}\left(S^{n} A^{*}\right)$ :

$$
\begin{gathered}
\chi_{n^{2}}(\gamma)=\gamma \otimes \ldots \otimes 1 \\
f_{1}\left(\chi_{n^{2}}(\gamma)\right)=\chi_{n^{2}}(\gamma)=\chi_{n}(\gamma) \otimes \underbrace{1 \otimes \ldots \otimes 1}_{1 \in S^{n} A^{*}} \otimes \ldots \otimes \underbrace{1 \otimes \ldots \otimes 1}_{1 \in S^{n} A^{*}}+\ldots \\
+\underbrace{1 \otimes \ldots \otimes 1}_{1 \in S^{n} A^{*}} \otimes \ldots \otimes \underbrace{1 \otimes \ldots \otimes 1}_{1 \in S^{n} A^{*}} \otimes \chi_{n}(\gamma) .
\end{gathered}
$$


Step $f_{2}$. The map $f_{2}: S^{n}\left(S^{n} A^{*}\right) \rightarrow S^{n}\left(A^{*} \otimes A^{*}\right)$ is induced by the map $\operatorname{Sym}^{n} \mu$ : $\operatorname{Sym}^{n}(X \times X) \rightarrow \operatorname{Sym}^{n}\left(\operatorname{Sym}^{n} X\right)$, which can be obtained from the commutative diagram

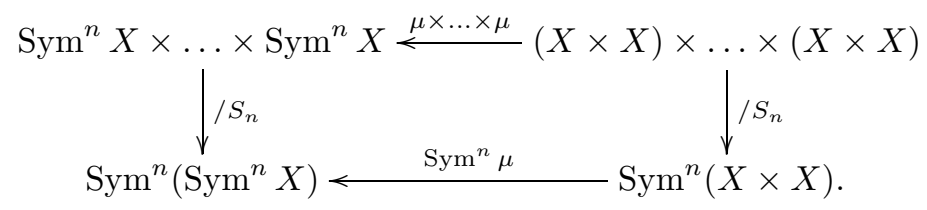

Passing to cohomology, we have a commutative diagram

$$
S^{n} A^{*} \otimes \ldots \otimes S^{n} A^{*} \stackrel{\mu^{*} \otimes \ldots \otimes \mu^{*}}{\longrightarrow}\left(A^{*} \otimes A^{*}\right) \otimes \ldots \otimes\left(A^{*} \otimes A^{*}\right)
$$

$\left(f_{2.2}\right)$

$$
S^{n}\left(S^{n} A^{*}\right) \longrightarrow S^{n}\left(A^{*} \otimes A^{*}\right) .
$$

Since both its vertical arrows are identity inclusions, the map $f_{2}$ coincides with the restriction of $\mu^{*} \otimes \ldots \otimes \mu^{*}$ to the pair $\left(S^{n}\left(S^{n} A^{*}\right), S^{n}\left(A^{*} \otimes A^{*}\right)\right)$. Now we determine $f_{2} \circ f_{1}\left(\chi_{n^{2}}(\gamma)\right) \in S^{n}\left(A^{*} \otimes A^{*}\right):$

$$
\begin{aligned}
& f_{2} \circ f_{1}\left(\chi_{n^{2}}(\gamma)\right)=\mu^{*} \otimes \ldots \otimes \mu^{*}(\chi_{n}(\gamma) \otimes \ldots \otimes \underbrace{1 \otimes \ldots \otimes 1}_{1 \in S^{n} A^{*}}+\ldots \\
& +\underbrace{1 \otimes \ldots \otimes 1}_{1 \in S^{n} A^{*}} \otimes \ldots \otimes \chi_{n}(\gamma)) \\
& =\Delta(\gamma) \otimes \underbrace{1 \otimes 1}_{1 \in A^{*} \otimes A^{*}} \otimes \ldots \otimes \underbrace{1 \otimes 1}_{1 \in A^{*} \otimes A^{*}}+\ldots+\underbrace{1 \otimes 1}_{1 \in A^{*} \otimes A^{*}} \otimes \ldots \otimes \underbrace{1 \otimes 1}_{1 \in A^{*} \otimes A^{*}} \otimes \Delta(\gamma) .
\end{aligned}
$$

This computation makes sense since $\mu^{*}: S^{n} A^{*} \rightarrow A^{*} \otimes A^{*}$ is an algebra homomorphism, and, by definition, $\mu^{*}\left(\chi_{n}(\gamma)\right)=\Delta(\gamma)$.

Step $f_{3}$. The map $f_{3}: S^{n}\left(A^{*} \otimes A^{*}\right) \rightarrow S^{n} A^{*} \otimes A^{*}$ is induced by the map $\pi_{(1 L)}: \operatorname{Sym}^{n} X$ $\times X \rightarrow \operatorname{Sym}^{n}(X \times X)$, which is determined by the commutative diagram

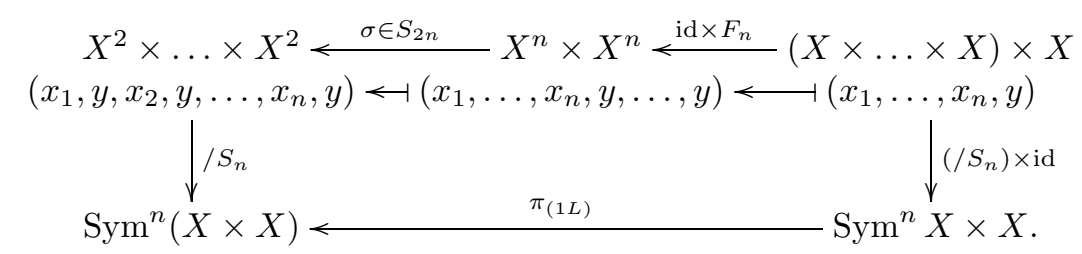

Passing to cohomology, we have:

$\left(f_{3.2}\right)$

$$
\left(A^{*} \otimes A^{*}\right) \otimes \ldots \otimes\left(A^{*} \otimes A^{*}\right) \stackrel{\sigma^{*}}{\longrightarrow} A^{\otimes n} \otimes A^{\otimes n} \stackrel{\mathrm{id} \otimes m}{\longrightarrow} A^{\otimes n} \otimes A^{*}
$$

$$
S^{n}\left(A^{*} \otimes A^{*}\right) \longrightarrow f_{3} A^{*} \otimes A^{*}
$$

Here, $m: A^{\otimes n} \rightarrow A^{*}$ denotes the algebra homomorphism corresponding to the multiplication on $A^{*}$. Since the vertical arrows of this diagram are identity inclusions, $f_{3}$ is the restriction of (id $\otimes m) \circ \sigma^{*}$ to the pair $\left(S^{n}\left(A^{*} \otimes A^{*}\right), S^{n} A^{*} \otimes A^{*}\right)$. Now we determine the values of $(\mathrm{id} \otimes m) \circ \sigma^{*}$ on the tensor

$$
\overline{\varepsilon \delta}_{(k)}=(1 \otimes 1) \otimes \ldots \otimes \underbrace{(\varepsilon \otimes \delta)}_{k \text {-th place }} \otimes \ldots \otimes(1 \otimes 1) \in\left(A^{*} \otimes A^{*}\right) \otimes \ldots \otimes\left(A^{*} \otimes A^{*}\right),
$$


$1 \leq k \leq n$. We have

$$
\begin{aligned}
& \sigma^{*}\left(\overline{\varepsilon \delta}_{(k)}\right)=(1 \otimes \ldots \otimes \underbrace{\varepsilon}_{k \text {-th place }} \otimes \ldots \otimes 1) \otimes(1 \otimes \ldots \otimes \underbrace{\delta}_{k \text {-th place }} \otimes \ldots \otimes 1) ; \\
& (\mathrm{id} \otimes m) \circ \sigma^{*}\left(\overline{\varepsilon \delta}_{(k)}\right)=(1 \otimes \ldots \otimes \underbrace{\varepsilon}_{k \text {-th place }} \otimes \ldots \otimes 1) \otimes \delta, \quad 1 \leq k \leq n .
\end{aligned}
$$

Using the decomposition $\Delta(\gamma)=\sum_{i=1}^{N} \alpha_{i} \otimes \beta_{i} \in A^{*} \otimes A^{*}$ and the explicit expression for $($ id $\otimes m) \circ \sigma^{*}\left(\overline{\varepsilon \delta}_{(k)}\right), 1 \leq k \leq n$, we have

$$
\begin{aligned}
f_{3} \circ f_{2} \circ f_{1}\left(\chi_{n^{2}}(\gamma)\right) & =f_{3}(\Delta(\gamma) \otimes \ldots \otimes(1 \otimes 1)+\ldots+(1 \otimes 1) \otimes \ldots \otimes \Delta(\gamma)) \\
& =\sum_{i=1}^{N} \chi_{n}\left(\alpha_{i}\right) \otimes \beta_{i} \in S^{n} A^{*} \otimes A^{*} .
\end{aligned}
$$

Step $f_{4}$. The last in the composition map $f_{4}: S^{n} A^{*} \otimes A^{*} \rightarrow A^{*} \otimes A^{*} \otimes A^{*}$ equals $\mu^{*} \otimes$ id. We now compute $f_{4} \circ f_{3} \circ f_{2} \circ f_{1}\left(\chi_{n^{2}}(\gamma)\right)$ :

$$
\begin{aligned}
f_{4} \circ f_{3} \circ f_{2} \circ f_{1}\left(\chi_{n^{2}}(\gamma)\right) & =\mu^{*} \otimes \mathrm{id}\left(\sum_{i=1}^{N} \chi_{n}\left(\alpha_{i}\right) \otimes \beta_{i}\right) \\
& =\sum_{i=1}^{N} \Delta\left(\alpha_{i}\right) \otimes \beta_{i} \in A^{*} \otimes A^{*} \otimes A^{*} .
\end{aligned}
$$

In summary, we have done the following. We fixed an arbitrary homogeneous element $\gamma \in A^{q}, q \geq 1$. Suppose $\Delta(\gamma) \in A^{*} \otimes A^{*}$ has a decomposition $\Delta(\gamma)=\sum_{i=1}^{N} \alpha_{i} \otimes \beta_{i}$. Then the image of $\chi_{n^{2}}(\gamma) \in S^{n^{2}} A^{*}$ under $f_{4} \circ f_{3} \circ f_{2} \circ f_{1}: S^{n^{2}} A^{*} \rightarrow A^{*} \otimes A^{*} \otimes A^{*}$ is $\sum_{i=1}^{N} \Delta\left(\alpha_{i}\right) \otimes \beta_{i}$, which is the image of $\gamma \in A^{*}$ under the composition $A^{*} \stackrel{\Delta}{\longrightarrow} A^{*} \otimes$ $A^{*} \stackrel{\Delta \otimes \mathrm{id}}{\longrightarrow} A^{*} \otimes A^{*} \otimes A^{*}$. Applying the same arguments (steps 1- 4), with obvious modifications, to diagram $\left(3^{\prime}\right)$, it is not difficult to see that $g_{4} \circ g_{3} \circ g_{2} \circ g_{1}\left(\chi_{n^{2}}(\gamma)\right)=$ $\sum_{i=1}^{N} \alpha_{i} \otimes \Delta\left(\beta_{i}\right) \in A^{*} \otimes A^{*} \otimes A^{*}$. Moreover, $\sum_{i=1}^{N} \alpha_{i} \otimes \Delta\left(\beta_{i}\right)$ is the image of $\gamma \in A^{*}$ under the composition $A^{*} \stackrel{\Delta}{\longrightarrow} A^{*} \otimes A^{*} \stackrel{\mathrm{id} \otimes \Delta}{\longrightarrow} A^{*} \otimes A^{*} \otimes A^{*}$.

By the assumption, the $n$-valued multiplication $\mu: X \times X \rightarrow \operatorname{Sym}^{n} X$ is associative, and, therefore, the compositions in diagrams (2) and (3) are both equal to $\mu_{(2)}: X \times X \times$ $X \rightarrow \operatorname{Sym}^{n^{2}} X$. Then, passing to cohomology, we have $f_{4} \circ f_{3} \circ f_{2} \circ f_{1}=g_{4} \circ g_{3} \circ g_{2} \circ g_{1}=$ $\mu_{(2)}^{*}: S^{n^{2}} A^{*} \rightarrow A^{*} \otimes A^{*} \otimes A^{*}$. In view of the previous paragraph, the maps

$$
A^{*} \stackrel{\Delta}{\longrightarrow} A^{*} \otimes A^{*} \stackrel{\Delta \otimes \mathrm{id}}{\longrightarrow} A^{*} \otimes A^{*} \otimes A^{*} \quad \text { and } \quad A^{*} \stackrel{\Delta}{\longrightarrow} A^{*} \otimes A^{*} \stackrel{\operatorname{id} \otimes \Delta}{\longrightarrow} A^{*} \otimes A^{*} \otimes A^{*}
$$

agree at least on the elements $\gamma \in A^{*}$ of positive degrees. We also see that $(\Delta \otimes \mathrm{id}) \circ \Delta(1)=$ $($ id $\otimes \Delta) \circ \Delta(1)=n^{2} 1 \otimes 1 \otimes 1$. Since $A^{*}$ is connected, $A^{0}=\mathbb{Q}$, and we have equality everywhere $(\Delta \otimes \mathrm{id}) \circ \Delta=(\mathrm{id} \otimes \Delta) \circ \Delta: A^{*} \rightarrow A^{*} \otimes A^{*} \otimes A^{*}$. This proves the coassociativity of the diagonal $\Delta: A^{*} \rightarrow A^{*} \otimes A^{*}$.

Inversion. Suppose our $n$-valued multiplication $\mu: X \times X \rightarrow \operatorname{Sym}^{n} X$ admits an inverse; i.e., there exists a continuous map inv: $X \rightarrow X$ such that $\operatorname{inv}(x) * x \ni e \forall x \in X$ and $x * \operatorname{inv}(x) \ni e \forall x \in X$. Then it is easy to see that there are continuous maps $F_{(n-1, L)}, F_{(n-1, R)}: X \rightarrow \operatorname{Sym}^{n-1} X$ such that $\operatorname{inv}(x) * x=\left[e, F_{(n-1, L)}(x)\right] \in \operatorname{Sym}^{n} X$ $\forall x \in X$ and $x * \operatorname{inv}(x)=\left[e, F_{(n-1, R)}(x)\right] \in \operatorname{Sym}^{n} X \forall x \in X$. This can be rewritten in 
terms of the commutative diagrams
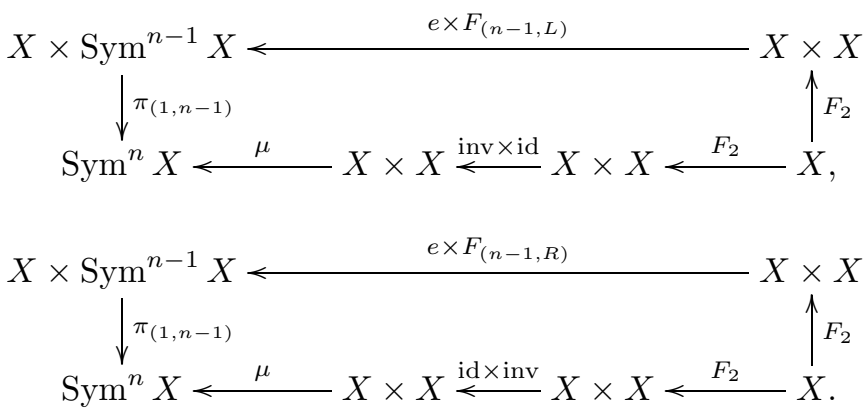

Here, $F_{2}: X \rightarrow X \times X, F_{2}(x)=(x, x) \forall x \in X$, denotes the standard diagonal, and $e: X \rightarrow X$ is the constant map, sending the entire $X$ to $e \in X$. Denote the algebra homomorphism inv ${ }^{*}: A^{*} \rightarrow A^{*}$ by $\chi$, and the $n$-homomorphisms $F_{(n-1, L)}^{*} \circ \chi_{n-1}: A^{*} \rightarrow$ $A^{*}$ and $F_{(n-1, R)}^{*} \circ \chi_{n-1}: A^{*} \rightarrow A^{*}$ by $\chi_{L}^{\perp}$ and $\chi_{R}^{\perp}$, respectively. Passing to cohomology in diagram (4), we have a commutative diagram:

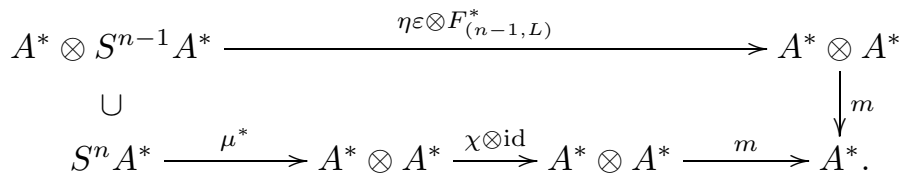

Here $e^{*}=\eta \circ \varepsilon:=\eta \varepsilon: A^{*} \rightarrow A^{*}$, where $\varepsilon: A^{*} \rightarrow \mathbb{Q}=A^{0}$ is the augmentation, and $\eta: \mathbb{Q} \rightarrow A^{*}$ is the algebra homomorphism defining the unit of $A^{*}$. The left vertical arrow in this diagram is the identity inclusion by virtue of the following commutative diagrams:

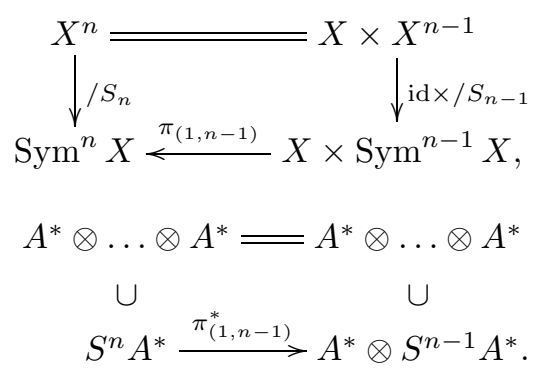

We now fix an arbitrary homogenous element $\gamma \in A^{*},|\gamma| \geq 1$. We want to determine where the element $\chi_{n}(\gamma)=\gamma \otimes \ldots \otimes 1+\ldots+1 \otimes \ldots \otimes \gamma \in S^{n} A^{*}$ is sent to by the arrows in diagram $\left(4^{\prime}\right)$.

Since $\mu^{*} \circ \chi_{n}=\Delta: A^{*} \rightarrow A^{*} \otimes A^{*}$, the element $\chi_{n}(\gamma)$, after passing the bottom row, becomes $m \circ(\chi \otimes \mathrm{id}) \circ \Delta(\gamma) \in A^{*}$. Under the inclusion $S^{n} A^{*} \subset A^{*} \otimes S^{n-1} A^{*}$, the element $\chi_{n}(\gamma)$ goes to $\gamma \otimes(1 \otimes \ldots \otimes 1)+1 \otimes \chi_{n-1}(\gamma)$. Since $\eta \varepsilon(\gamma)=0$ and $F_{(n-1, L)}^{*} \circ \chi_{n-1}(\gamma)=$ $\chi_{L}^{\frac{1}{L}}(\gamma)$, the element $\gamma \otimes(1 \otimes \ldots \otimes 1)+1 \otimes \chi_{n-1}(\gamma)$, after passing the top row and the right vertical arrow in diagram $\left(4^{\prime}\right)$, becomes $\chi_{L}^{\frac{1}{L}}(\gamma) \in A^{*}$. By the commutativity of the diagram, the $n$-homomorphisms $m \circ(\chi \otimes \mathrm{id}) \circ \Delta: A^{*} \rightarrow A^{*}$ and $\eta \varepsilon+\chi_{L}^{\perp}: A^{*} \rightarrow A^{*}$ agree at least on the elements $\gamma \in A^{*}$ of positive degree.

It is clear that $m \circ(\chi \otimes \mathrm{id}) \circ \Delta(1)=n=\left(\eta \varepsilon+\chi_{L}^{\perp}\right)(1)$. Therefore, we have the desired equality $m \circ(\chi \otimes \mathrm{id}) \circ \Delta=\eta \varepsilon+\chi_{L}^{\perp}$ of $n$-homomorphisms. Applying similar arguments to diagram (5), we have $m \circ($ id $\otimes \chi) \circ \Delta=\eta \varepsilon+\chi_{R}^{\perp}$. Thus, we have proved that the diagonal $\Delta: A^{*} \rightarrow A^{*} \otimes A^{*}$ has the inversion property.

The theorem has been proved. 
Now, we want to introduce a homotopical analog of $n$-valued topological groups. Henceforth, all spaces, maps and homotopies will be relative to a base point. For a path-connected Hausdorff space $X$ with base point $e \in X$, we define an $n$-valued multiplication on $X$ as a continuous map $\mu:(X \times X,(e, e)) \rightarrow\left(\operatorname{Sym}^{n} X,[n e]\right)$. As before, we set $\mu(x, y)=x * y \forall x, y \in X$.

Definition 3.1.6. The pair $(X, \mu)$ is called an $n H$-space if the following homotopy unit axiom holds:

(1) The maps $\mu(x, e): X \rightarrow \operatorname{Sym}^{n} X$ and $\mu(e, x): X \rightarrow \operatorname{Sym}^{n} X$ are homotopic to the diagonal map $\Delta: X \rightarrow \operatorname{Sym}^{n} X, \Delta(x)=[n x] \forall x \in X$.

The pair $(X, \mu)$ is called an $n H$-monoid if, in addition to axiom (1), a homotopy associativity axiom also holds:

(2) The following maps are homotopic:

$$
\begin{aligned}
\operatorname{Sym}^{n^{2}} X \stackrel{\pi_{(2)}}{\longleftarrow} \operatorname{Sym}^{n}\left(\operatorname{Sym}^{n} X\right) \stackrel{\operatorname{Sym}^{n} \mu}{\longleftarrow} \operatorname{Sym}^{n}(X \times X) \\
\stackrel{\pi_{(1 L)}}{\longleftarrow} \operatorname{Sym}^{n} X \times X \stackrel{\mu \times \mathrm{id}}{\longleftarrow} X \times X \times X, \\
\operatorname{Sym}^{n^{2}} X \stackrel{\pi_{(2)}}{\longleftarrow} \operatorname{Sym}^{n}\left(\operatorname{Sym}^{n} X\right) \stackrel{\operatorname{Sym}^{n} \mu}{\longleftarrow} \operatorname{Sym}^{n}(X \times X) \\
\stackrel{\pi_{(1 R)}}{\longleftarrow} \times \operatorname{Sym}^{n} X \stackrel{\text { id } \times \mu}{\longleftarrow} X \times X \times X,
\end{aligned}
$$

where $\pi_{(1 L)}, \pi_{(1 R)}, \pi_{(2)}$ are the canonical projections given by

$$
\begin{gathered}
\pi_{(1 L)}\left(\left[x_{1}, \ldots, x_{n}\right], y\right)=\left[\left(x_{1}, y\right), \ldots,\left(x_{n}, y\right)\right], \\
\pi_{(1 R)}\left(y,\left[x_{1}, \ldots, x_{n}\right]\right)=\left[\left(y, x_{1}\right), \ldots,\left(y, x_{n}\right)\right], \\
\pi_{(2)}\left[\left[x_{11}, \ldots, x_{1 n}\right], \ldots,\left[x_{n 1}, \ldots, x_{n n}\right]\right]=\left[x_{11}, \ldots, x_{n n}\right] .
\end{gathered}
$$

For a given continuous map inv: $X \rightarrow X$, the triple $(X, \mu$, inv) is called an $n H$-group if, in addition to axioms (1) and (2), a homotopy inversion axiom holds:

(3) the maps $\mu(\operatorname{inv}(x), x): X \rightarrow \operatorname{Sym}^{n} X$ and $\mu(x, \operatorname{inv}(x)): X \rightarrow \operatorname{Sym}^{n} X$ are homotopic to the maps $X \rightarrow \operatorname{Sym}^{n} X$ of the form $(x \mapsto[e, F(x)])$, where $F: X \rightarrow \operatorname{Sym}^{n-1} X$ is some map.

Notice that the given definition differs from Definition 3.1.1 only in one aspect: the equality of the maps involved is replaced by their homotopy. But, as is easy to see, in the proof of Theorem 3.1.5 we only used the fact that equal (by Definition 3.1.1) maps induce the same homomorphism in cohomology. But homotopic maps also induce the same homomorphism in cohomology. It follows that the proof of Theorem 3.1.5 works equally well in the case when $X$ is an $n H$-space ( $n H$ monoid, $n H$-group).

Thus we have

Theorem 3.1.7. Suppose $X$ is a connected locally contractible paracompact space such that $X^{n^{2}}$ is also paracompact and $\operatorname{dim} H^{q}(X ; \mathbb{Q})<\infty \forall q \geq 0$. If $X$ has a structure of an $n H$-space ( $n H$-monoid, $n H$-group), then $H^{*}(X ; \mathbb{Q})$ has a structure of a graded $n$-Hopf prealgebra (graded n-bialgebra, graded $n$-Hopf algebra).

3.2. $2 H$-spaces and the fundamental group. As is well known, the fundamental group of any $H$-space is abelian. Moreover, for any abelian group $A$ there exists an $H$ group $K(A, 1)=\Omega K(A, 2)$ with fundamental group $A$. Thus, the question which groups can be fundamental groups of $H$-spaces has a simple answer: abelian groups and only abelian groups. As we shall see below, there are $2 H$-spaces (or even $2 H$-groups) with nonabelian fundamental groups. However, and this is the main result of Section 3, not any nonabelian group can be the fundamental group of a $2 \mathrm{H}$-space: there is a cohomological restriction. 
Recall that the cohomology of a discrete group $G$ is, by definition, the cohomology of its classifying space $B G=K(G, 1)$. We will only work with finitely presented groups $G$, i.e., with groups given by a finite number of generators and relations. It is well known that for any finitely presented group $G$ one can choose a cellular realization of $B G$ such that the 2 -skeleton $\mathrm{Sk}^{2} B G$ is finite. It now follows that $H_{1}(G ; \mathbb{Z})$ and $H_{2}(G ; \mathbb{Z})$ are finitely generated abelian groups, and, in particular, $H^{1}(G ; \mathbb{Q})$ and $H^{2}(G ; \mathbb{Q})$ are finite-dimensional.

Definition 3.2.1. A finitely presented group $G$ belongs to the class $\mathcal{C}$ if there exist a basis $\alpha_{1}, \alpha_{2}, \ldots, \alpha_{2 g-1}, \alpha_{2 g}, \beta_{1}, \ldots, \beta_{s}$ of $H^{1}(G ; \mathbb{Q}), g \geq 2, s \geq 0$, and a nonzero element $\gamma \in H^{2}(G ; \mathbb{Q})$ such that:

(1) $\alpha_{2 k-1} \alpha_{2 k}=-\alpha_{2 k} \alpha_{2 k-1}=\gamma \forall 1 \leq k \leq g$, and the remaining products $\alpha_{i} \alpha_{j}$ are zero;

(2) the linear span $L:=\left\langle\alpha_{i} \beta_{j}, 1 \leq i \leq 2 g, 1 \leq j \leq s ; \beta_{k} \beta_{l}, 1 \leq k, l \leq s\right\rangle$ does not contain the vector $\gamma$.

The next two propositions show that the class $\mathcal{C}$ is rather big.

Proposition 3.2.2. Fundamental groups of compact Riemann surfaces of genus $g \geq 2$ belong to $\mathcal{C}$.

Proof. Suppose $\Gamma_{g}$ is a compact Riemann surface of genus $g \geq 2$. This is an aspherical manifold; hence $\Gamma_{g}=B\left(\pi_{1} \Gamma_{g}\right)$. It is known that one can choose a basis $\alpha_{1}, \ldots, \alpha_{2 g}$ in $H^{1}\left(\Gamma_{g} ; \mathbb{Q}\right)$ such that condition (1) of Definition 3.2.1 is satisfied, where $\gamma$ is the fundamental cohomological class of $\Gamma_{g}$. Condition (2) of Definition 3.2.1 is satisfied automatically, since there are no elements $\beta_{i}$ (as $\left.s=0\right)$. Thus $\pi_{1} \Gamma_{g} \in \mathcal{C}$.

Proposition 3.2.3. For any group $G \in \mathcal{C}$ and any finitely presented group $K$, we have:

(1) $G \times K \in \mathcal{C}$;

(2) $G * K \in \mathcal{C}$.

Proof. (1) The Künneth formula for homology with coefficients in a field says the following ([18, Ch. VII, Prop. 2.6]). For any topological spaces $X$ and $Y$ and any field $K$, the $\times$-product in homology $\nu: H_{*}(X ; K) \otimes H_{*}(Y ; K) \rightarrow H_{*}(X \times Y ; K)$ is an isomorphism. A weaker version of this statement holds for cohomology ([18, p. 267, Ex. 7.15]): the $\times$-product in cohomology $\mu: H^{*}(X ; K) \otimes H^{*}(Y ; K) \rightarrow H^{*}(X \times Y ; K)$ is an algebra monomorphism (it is an epimorphism if and only if either $\operatorname{dim} H^{q}(X ; K)<\infty$ $\forall q \geq 0$, or $\left.\operatorname{dim} H^{q}(Y ; K)<\infty \forall q \geq 0\right)$. Assume now that $X$ and $Y$ are pathconnected and the homology groups of both spaces are finite-dimensional up to dimension $n, \operatorname{dim} H_{m}(X ; K)<\infty, \operatorname{dim} H_{m}(Y ; K)<\infty, 0 \leq m \leq n$. Since the pairing between homology and cohomology makes the $m$-th cohomology group with coefficients in a field into the dual space of the $m$-th homology group, for any $0 \leq m \leq n$ we have

$$
\begin{aligned}
\operatorname{dim}\left(H^{*}(X ; K) \otimes H^{*}(Y ; K)\right)^{m} & =\operatorname{dim}\left(H_{*}(X ; K) \otimes H_{*}(Y ; K)\right)_{m} \\
& =\operatorname{dim} H_{m}(X \times Y ; K)=\operatorname{dim} H^{m}(X \times Y ; K)<\infty .
\end{aligned}
$$

It now follows that the monomorphism $\mu: H^{*}(X ; K) \otimes H^{*}(Y ; K) \rightarrow H^{*}(X \times Y ; K)$ is an isomorphism up to dimension $n$.

Consider now the rational cohomology of the classifying spaces $B G, B K$, and $B(G \times K)$. Since $B G \times B K=B(G \times K)$, we have an algebra monomorphism $\mu$ : $H^{*}(G ; \mathbb{Q}) \otimes H^{*}(K ; \mathbb{Q}) \rightarrow H^{*}(G \times K ; \mathbb{Q})$. By the just used arguments, $\mu$ is an isomorphism at least in degrees 1 and 2 . By the assumption, $G$ belongs to $\mathcal{C}$. Thus, there are a basis $\alpha_{1}, \alpha_{2}, \ldots, \alpha_{2 g-1}, \alpha_{2 g}, \beta_{1}, \ldots, \beta_{s}, g \geq 2$, in $H^{1}(G ; \mathbb{Q})$ and a nonzero element $\gamma \in H^{2}(G ; \mathbb{Q})$ such that both conditions of Definition 3.2.1 are satisfied. Let $\delta_{1}, \ldots, \delta_{t}$ be an arbitrary basis in $H^{1}(K ; \mathbb{Q})$. By the above, the vectors $\alpha_{1} \otimes 1, \ldots, \alpha_{2 g} \otimes 1, \beta_{1} \otimes 1$, 
$\ldots, \beta_{s} \otimes 1,1 \otimes \delta_{1}, \ldots, 1 \otimes \delta_{t}$ form a basis in $H^{1}(G \times K ; \mathbb{Q})$. We shall show that this basis and the element $\gamma \otimes 1 \in H^{2}(G \times K ; \mathbb{Q})$ satisfy the conditions of Definition 3.2.1.

Indeed, $\alpha_{2 k-1} \otimes 1 \cdot \alpha_{2 k} \otimes 1=-\alpha_{2 k} \otimes 1 \cdot \alpha_{2 k-1} \otimes 1=\left(\alpha_{2 k-1} \alpha_{2 k}\right) \otimes 1=\gamma \otimes 1 \forall 1 \leq k \leq g$. The remaining products $\alpha_{i} \otimes 1 \cdot \alpha_{j} \otimes 1$ are all zero. We also have

$$
\begin{gathered}
\alpha_{i} \otimes 1 \cdot \beta_{j} \otimes 1=\alpha_{i} \beta_{j} \otimes 1, \quad 1 \leq i \leq 2 g, 1 \leq j \leq s \\
\alpha_{i} \otimes 1 \cdot 1 \otimes \delta_{j}=\alpha_{i} \otimes \delta_{j}, \quad 1 \leq i \leq 2 g, 1 \leq j \leq t ; \\
\beta_{k} \otimes 1 \cdot \beta_{l} \otimes 1=\beta_{k} \beta_{l} \otimes 1, \quad 1 \leq k, l \leq s \\
\beta_{k} \otimes 1 \cdot 1 \otimes \delta_{l}=\beta_{k} \otimes \delta_{l}, \quad 1 \leq k \leq s, 1 \leq l \leq t \\
1 \otimes \delta_{k} \cdot 1 \otimes \delta_{l}=1 \otimes \delta_{k} \delta_{l}, \quad 1 \leq k, l \leq t .
\end{gathered}
$$

Let $L$ be the linear span $L=\left\langle\alpha_{i} \beta_{j}, \beta_{k} \beta_{l}\right\rangle \subset H^{2}(G ; \mathbb{Q})$. Suppose also $L^{\prime}=\left\langle\alpha_{i} \beta_{j} \otimes\right.$ $\left.1, \alpha_{i} \otimes \delta_{j}, \beta_{k} \beta_{l} \otimes 1, \beta_{k} \otimes \delta_{l}, 1 \otimes \delta_{k} \delta_{l}\right\rangle$. By the Künneth formula,

$$
H^{2}(G \times K ; \mathbb{Q})=H^{2}(G ; \mathbb{Q}) \otimes\langle 1\rangle \oplus H^{1}(G ; \mathbb{Q}) \otimes H^{1}(K ; \mathbb{Q}) \oplus\langle 1\rangle \otimes H^{2}(K ; \mathbb{Q}) .
$$

It is easy to see that

$$
L^{\prime} \subset(L \otimes\langle 1\rangle) \oplus H^{1}(G ; \mathbb{Q}) \otimes H^{1}(K ; \mathbb{Q}) \oplus\langle 1\rangle \otimes H^{2}(K ; \mathbb{Q}) .
$$

Since $\gamma \otimes 1$ is not in $L \otimes\langle 1\rangle$, we have that $\gamma \otimes 1$ is not $L^{\prime}$. Thus, all conditions of Definition 3.2.1 are satisfied. Claim (1) is proved.

(2) As is well known, $B(G * K)=B G \vee B K$. The cohomology ring of the wedge product of two connected spaces has the following structure:

$$
H^{*}(X \vee Y ; \mathbb{Q})=\mathbb{Q}\langle 1\rangle \oplus\left(H^{1}(X ; \mathbb{Q}) \oplus H^{2}(X ; \mathbb{Q}) \oplus \ldots\right) \oplus\left(H^{1}(Y ; \mathbb{Q}) \oplus H^{2}(Y ; \mathbb{Q}) \oplus \ldots\right)
$$

Moreover, the multiplication in $\widetilde{H}^{*}(X \vee Y ; \mathbb{Q})=\widetilde{H}^{*}(X ; \mathbb{Q}) \oplus \widetilde{H}^{*}(Y ; \mathbb{Q})$ coincides with the multiplication in the direct sum of the rings.

Thus, $H^{*}(G * K ; \mathbb{Q})=\mathbb{Q}\langle 1\rangle \oplus\left(\widetilde{H}^{*}(G ; \mathbb{Q}) \oplus \widetilde{H}^{*}(K ; \mathbb{Q})\right)$. Consider the basis $\alpha_{1}, \ldots, \alpha_{2 g}$, $\beta_{1}, \ldots, \beta_{s} \in H^{1}(G ; \mathbb{Q})$ and the element $\gamma \in H^{2}(G ; \mathbb{Q})$ from Definition 3.2.1. Let $\delta_{1}, \ldots, \delta_{t}$ be an arbitrary basis in $H^{1}(K ; \mathbb{Q})$.

Thus, we have a basis $\alpha_{1}, \ldots, \alpha_{2 g}, \beta_{1}, \ldots, \beta_{s}, \delta_{1}, \ldots, \delta_{t}$ in $H^{1}(G * K ; \mathbb{Q})$ and a nonzero element $\gamma \in H^{2}(G * K ; \mathbb{Q})$. We want to check that these elements satisfy the conditions in the definition of $\mathcal{C}$. Condition (1) holds automatically. For the second condition, we have five sets of elements: $\alpha_{i} \beta_{j}, \alpha_{i} \delta_{j}, \beta_{i} \beta_{j}, \beta_{i} \delta_{j}, \delta_{i} \delta_{j}$. It is clear that $\alpha_{i} \delta_{j}=0$, $1 \leq i \leq 2 g, 1 \leq j \leq t$, and $\beta_{i} \delta_{j}=0,1 \leq i \leq s, 1 \leq j \leq t$. We set $L=\left\langle\alpha_{i} \beta_{j}, \beta_{i} \beta_{j}\right\rangle$ and $L^{\prime}=\left\langle\alpha_{i} \beta_{j}, \alpha_{i} \delta_{j}, \beta_{i} \beta_{j}, \beta_{i} \delta_{j}, \delta_{i} \delta_{j}\right\rangle$. Then $H^{2}(G * K ; \mathbb{Q})=H^{2}(G ; \mathbb{Q}) \oplus H^{2}(K ; \mathbb{Q})$ and $L^{\prime} \subset L \oplus H^{2}(K ; \mathbb{Q})$. Since $\gamma$ is not in $L$, we have that $\gamma$ is not in $L^{\prime}$. Thus, $G * K \in \mathcal{C}$, and the proposition is proved.

The following theorem is the main result of Section 3 .

Theorem 3.2.4. Let $X$ be a connected countable $C W$-complex such that $\operatorname{dim} H^{q}(X ; \mathbb{Q})$ $<\infty \forall q \geq 0$. If $X$ admits a structure of a $2 H$-space, then its fundamental group does not belong to the class $\mathcal{C}$.

Proof. Let $\pi_{1} X=G$. Consider a cellular realization of $B G$. The homotopy theory of cellular complexes tells us that for any isomorphism $f_{*}: \pi_{1}\left(X, x_{0}\right) \rightarrow G=\pi_{1}\left(B G, b_{0}\right)$ there exists a unique, up to a homotopy of based spaces, map $f:\left(X, x_{0}\right) \rightarrow\left(B G, b_{0}\right)$ realizing this isomorphism. Such maps are said to be characteristic. Fix an isomorphism $f_{*}: \pi_{1}\left(X, x_{0}\right) \rightarrow G=\pi_{1}\left(B G, b_{0}\right)$ and a characteristic map $f:\left(X, x_{0}\right) \rightarrow\left(B G, b_{0}\right)$. Since $f_{*}: \pi_{1} X \rightarrow G$ is an isomorphism, the map $f_{*}: H_{1}(X ; \mathbb{Z}) \rightarrow H_{1}(B G ; \mathbb{Z})$ is also an isomorphism. It now follows that both $f_{*}: H_{1}(X ; \mathbb{Q}) \rightarrow H_{1}(B G ; \mathbb{Q})$ and $f^{*}: H^{1}(B G ; \mathbb{Q}) \rightarrow$ $H^{1}(X ; \mathbb{Q})$ are isomorphisms. 
A famous theorem of Hopf says that the map $f_{*}: H_{2}(X ; \mathbb{Z}) \rightarrow H_{2}(B G ; \mathbb{Z})$ is an epimorphism (whose kernel is the image of the Hurewicz homomorphism $h: \pi_{2} X \rightarrow H_{2}(X ; \mathbb{Z})$ ). Therefore, $f_{*}: H_{2}(X ; \mathbb{Q}) \rightarrow H_{2}(B G ; \mathbb{Q})$ is also an epimorphism, and $f^{*}: H^{2}(B G ; \mathbb{Q}) \rightarrow$ $H^{2}(X ; \mathbb{Q})$ is, respectively, a monomorphism.

Suppose now that $G \in \mathcal{C}$. Then there are a basis $\alpha_{1}, \ldots, \alpha_{2 g}, \beta_{1}, \ldots, \beta_{s} \in H^{1}(B G ; \mathbb{Q})$, $g \geq 2$, and a nonzero element $\gamma \in H^{2}(B G ; \mathbb{Q})$ such that:

(1) $\alpha_{2 k-1} \alpha_{2 k}=-\alpha_{2 k} \alpha_{2 k-1}=\gamma, 1 \leq k \leq g$, and the remaining products $\alpha_{i} \alpha_{j}$ are zero;

(2) the linear span $\left\langle\alpha_{i} \beta_{j}, \beta_{k} \beta_{l}\right\rangle$ does not contain the vector $\gamma$.

Since the algebra homomorphism $f^{*}: H^{*}(B G ; \mathbb{Q}) \rightarrow H^{*}(X ; \mathbb{Q})$ is an isomorphism in dimension 1 and a monomorphism in dimension 2 , we can identify $\alpha_{1}, \ldots, \alpha_{2 g}, \beta_{1}, \ldots, \beta_{s}$ and $\gamma$ with their images in $H^{1}(X ; \mathbb{Q})$ and $H^{2}(X ; \mathbb{Q})$. Moreover, the new elements $\alpha_{1}, \ldots, \alpha_{2 g}, \beta_{1}, \ldots, \beta_{s} \in H^{1}(X ; \mathbb{Q})$ and $\gamma \in H^{2}(X ; \mathbb{Q})$ satisfy the same conditions $(1)$ and (2). Now we can forget about the fundamental group $\pi_{1} X$ and the space $B G$, and concentrate only on these distinguished elements $\alpha_{1}, \ldots, \alpha_{2 g}, \beta_{1}, \ldots, \beta_{s} \in H^{1}(X ; \mathbb{Q})$ and $\gamma \in H^{2}(X ; \mathbb{Q})$.

To prove the theorem we need to show that $X$ does not admit a structure of a $2 \mathrm{H}$ space. Assume the opposite. Since a finite product of countable $C W$-complexes is a $C W$-complex, and since $C W$-complexes are locally contractible and paracompact, we can apply Theorem 3.1.7, which implies that there exists a diagonal $\Delta: H^{*}(X ; \mathbb{Q}) \rightarrow$ $H^{*}(X ; \mathbb{Q}) \otimes H^{*}(X ; \mathbb{Q})$, giving rise to a structure of a graded 2-Hopf prealgebra on $H^{*}(X ; \mathbb{Q})$. Let $A^{*}$ denote the algebra $H^{*}(X ; \mathbb{Q})$. Since the diagonal $\Delta: A^{*} \rightarrow A^{*} \otimes A^{*}$ satisfies the counit axiom, we have:

$$
\begin{gathered}
\Delta\left(\alpha_{i}\right)=2\left(\alpha_{i} \otimes 1+1 \otimes \alpha_{i}\right), \quad 1 \leq i \leq 2 g \\
\Delta\left(\beta_{j}\right)=2\left(\beta_{j} \otimes 1+1 \otimes \beta_{j}\right), \quad 1 \leq j \leq s \\
\Delta(\gamma)=2 \gamma \otimes 1+1 \otimes 2 \gamma+2 d^{s t} \alpha_{s} \otimes \alpha_{t}+2 \nu^{s t} \alpha_{s} \otimes \beta_{t}+2 \mu^{s t} \beta_{s} \otimes \alpha_{t}+2 \varepsilon^{s t} \beta_{s} \otimes \beta_{t} .
\end{gathered}
$$

Since $\Delta$ is a 2-homomorphism, we should have $\Phi_{3}(\Delta)\left(a_{1}, a_{2}, a_{3}\right)=0$ for any $a_{1}, a_{2}, a_{3} \in$ $A^{*}$. We examine this equality in two cases:

(A) $a_{1}=\alpha_{i}, a_{2}=\alpha_{j}, a_{3}=\alpha_{k}, 1 \leq i, j, k \leq 2 g$;

(B) $a_{1}=\alpha_{1}, a_{2}=\alpha_{2}, a_{3}=\gamma$.

In both cases $\left|a_{1}\right|=\left|a_{2}\right|=1$. Expanding $\Phi_{3}(\Delta)\left(a_{1}, a_{2}, a_{3}\right)$ via the Frobenius recursion, we have

$$
\begin{aligned}
\Phi_{3}(\Delta)\left(a_{1}, a_{2}, a_{3}\right)= & \Delta\left(a_{1}\right) \Phi_{2}(\Delta)\left(a_{2}, a_{3}\right)-\Phi_{2}(\Delta)\left(a_{1} a_{2}, a_{3}\right) \\
& -(-1)^{\left|a_{1} \| a_{2}\right|=1} \Phi_{2}(\Delta)\left(a_{2}, a_{1} a_{3}\right) \\
= & \Delta\left(a_{1}\right)\left(\Delta\left(a_{2}\right) \Delta\left(a_{3}\right)-\Delta\left(a_{2} a_{3}\right)\right) \\
& -\left(\Delta\left(a_{1} a_{2}\right) \Delta\left(a_{3}\right)-\Delta\left(a_{1} a_{2} a_{3}\right)\right)+\left(\Delta\left(a_{2}\right) \Delta\left(a_{1} a_{3}\right)-\Delta\left(a_{2} a_{1} a_{3}\right)\right) \\
= & \Delta\left(a_{1}\right) \Delta\left(a_{2}\right) \Delta\left(a_{3}\right)-\Delta\left(a_{1}\right) \Delta\left(a_{2} a_{3}\right)-\Delta\left(a_{1} a_{2}\right) \Delta\left(a_{3}\right) \\
& +\Delta\left(a_{2}\right) \Delta\left(a_{1} a_{3}\right)+\Delta\left(a_{1} a_{2} a_{3}\right)-\Delta\left(a_{2} a_{1} a_{3}\right) .
\end{aligned}
$$

Notice that $\alpha_{i} \gamma=0 \forall 1 \leq i \leq 2 g$. Indeed, for any $i, 1 \leq i \leq 2 g$, there exists $j$ (equal to $i \pm 1$ ), such that $\alpha_{i} \gamma= \pm \alpha_{i} \alpha_{i} \alpha_{j}=0$, because $\alpha \alpha=0 \forall \alpha \in A^{1}$. This implies that $\alpha_{i} \alpha_{j} \alpha_{k}=0 \forall 1 \leq i, j, k \leq 2 g$ and $\gamma^{2}=0$. Therefore, in both cases we have $a_{1} a_{2} a_{3}=a_{2} a_{1} a_{3}=0$. Moreover, in the first case, $a_{1} a_{3}=(-1)^{\left|a_{1}\right|\left|a_{3}\right|=1} a_{3} a_{1}=-a_{3} a_{1}$. In the second case, we have $a_{1} a_{3}=0=-a_{3} a_{1}$. In both cases we also have $\left|a_{1} a_{2}\right|=2$, and therefore $\Delta\left(a_{1} a_{2}\right) \Delta\left(a_{3}\right)=\Delta\left(a_{3}\right) \Delta\left(a_{1} a_{2}\right)$. 
In summary, in both cases we have the following expression for $\Phi_{3}(\Delta)\left(a_{1}, a_{2}, a_{3}\right)$ :

$$
\begin{aligned}
\Phi_{3}(\Delta)\left(a_{1}, a_{2}, a_{3}\right)=\Delta\left(a_{1}\right) \Delta & \left(a_{2}\right) \Delta\left(a_{3}\right) \\
& -\Delta\left(a_{1}\right) \Delta\left(a_{2} a_{3}\right)-\Delta\left(a_{2}\right) \Delta\left(a_{3} a_{1}\right)-\Delta\left(a_{3}\right) \Delta\left(a_{1} a_{2}\right) .
\end{aligned}
$$

Setting $f=\frac{1}{2} \Delta: A^{*} \rightarrow A^{*} \otimes A^{*}$, we have

$$
\begin{gathered}
f\left(\alpha_{i}\right)=\alpha_{i} \otimes 1+1 \otimes \alpha_{i}, \quad 1 \leq i \leq 2 g \\
f\left(\beta_{j}\right)=\beta_{j} \otimes 1+1 \otimes \beta_{j}, \quad 1 \leq j \leq s ; \\
f(\gamma)=\gamma \otimes 1+1 \otimes \gamma+d^{s t} \alpha_{s} \otimes \alpha_{t}+\nu^{s t} \alpha_{s} \otimes \beta_{t}+\mu^{s t} \beta_{s} \otimes \alpha_{t}+\varepsilon^{s t} \beta_{s} \otimes \beta_{t} .
\end{gathered}
$$

By formula (3), in both cases our identity $\Phi_{3}(\Delta)\left(a_{1}, a_{2}, a_{3}\right)=0$ is equivalent to the following identity:

$$
2 f\left(a_{1}\right) f\left(a_{2}\right) f\left(a_{3}\right)-f\left(a_{1}\right) f\left(a_{2} a_{3}\right)-f\left(a_{2}\right) f\left(a_{3} a_{1}\right)-f\left(a_{3}\right) f\left(a_{1} a_{2}\right)=0 .
$$

Now define a matrix $C=\left(c_{i j}\right)$ by setting $\alpha_{i} \alpha_{j}=c_{i j} \gamma, 1 \leq i, j \leq 2 g$, and consider our two cases.

CASE A. Here we want to show that (5) implies $D:=\left(d^{s t}\right)=C^{-1}$. Suppose $a_{1}=\alpha_{i}$, $a_{2}=\alpha_{j}, a_{3}=\alpha_{k}, i, j, k=1, \ldots, 2 g$. A direct calculation shows that

$$
\begin{aligned}
& f\left(a_{1}\right) f\left(a_{2}\right) f\left(a_{3}\right)=\left(\alpha_{i} \otimes 1+1 \otimes \alpha_{i}\right)\left(\alpha_{j} \otimes 1+1 \otimes \alpha_{j}\right)\left(\alpha_{k} \otimes 1+1 \otimes \alpha_{k}\right) \\
& =c_{i j}\left(\gamma \otimes \alpha_{k}+\alpha_{k} \otimes \gamma\right)+c_{j k}\left(\gamma \otimes \alpha_{i}+\alpha_{i} \otimes \gamma\right)+c_{k i}\left(\gamma \otimes \alpha_{j}+\alpha_{j} \otimes \gamma\right),
\end{aligned}
$$

whence

$$
2 f\left(a_{1}\right) f\left(a_{2}\right) f\left(a_{3}\right)=2\left(c_{i j} \delta_{k}^{n}+c_{j k} \delta_{i}^{n}+c_{k i} \delta_{j}^{n}\right)\left(\gamma \otimes \alpha_{n}+\alpha_{n} \otimes \gamma\right) .
$$

Now examine the value $f\left(a_{1}\right) f\left(a_{2} a_{3}\right)=f\left(\alpha_{i}\right) f\left(\alpha_{j} \alpha_{k}\right)$. A direct calculation shows that

$$
\begin{aligned}
f\left(\alpha_{i}\right) f\left(\alpha_{j} \alpha_{k}\right)= & f\left(\alpha_{i}\right) f\left(c_{j k} \gamma\right)=c_{j k}\left(\alpha_{i} \otimes 1+1 \otimes \alpha_{i}\right) f(\gamma) \\
= & c_{j k}\left(\delta_{i}^{n}+c_{i m} d^{m n}\right) \gamma \otimes \alpha_{n}+c_{j k}\left(\delta_{i}^{n}+d^{n m} c_{m i}\right) \alpha_{n} \otimes \gamma \\
& \quad+r_{(\gamma \beta)}^{s} \gamma \otimes \beta_{s}+r_{(\beta \gamma)}^{s} \beta_{s} \otimes \gamma+r_{(\alpha \beta, \alpha)}^{s t} \alpha_{i} \beta_{s} \otimes \alpha_{t} \\
& \quad+r_{(\alpha, \alpha \beta)}^{s t} \alpha_{s} \otimes \alpha_{i} \beta_{t}+r_{(\alpha \beta, \beta)}^{s t} \alpha_{i} \beta_{s} \otimes \beta_{t}+r_{(\beta, \alpha \beta)}^{s t} \beta_{s} \otimes \alpha_{i} \beta_{t} .
\end{aligned}
$$

In this formula, the last 6 groups of summands are the remainder term, with $r_{*}^{*}$ some constants whose exact form is not important.

Set $L=\left\langle\alpha_{s} \beta_{t}, \beta_{p} \beta_{q}\right\rangle \subset A^{2}$, and let $w_{1}, \ldots, w_{N}$ be an arbitrary basis in $L$. Now extend the linearly independent vectors $\gamma, w_{1}, \ldots, w_{N}$ to a basis $\gamma, w_{1}, \ldots, w_{N}, u_{1}, \ldots, u_{N^{\prime}}$ of $A^{2}$. Thus, formula (7) can be rewritten as

$$
\begin{array}{r}
f\left(\alpha_{i}\right) f\left(\alpha_{j} \alpha_{k}\right)=c_{j k}\left(\delta_{i}^{n}+c_{i m} d^{m n}\right) \gamma \otimes \alpha_{n}+c_{j k}\left(\delta_{i}^{n}+d^{n m} c_{m i}\right) \alpha_{n} \otimes \gamma+r_{(\gamma \beta)}^{s} \gamma \otimes \beta_{s} \\
\quad+r_{(\beta \gamma)}^{s} \beta_{s} \otimes \gamma+r_{(w \alpha)}^{s t} w_{s} \otimes \alpha_{t}+r_{(\alpha w)}^{t s} \alpha_{t} \otimes w_{s}+r_{(w \beta)}^{s t} w_{s} \otimes \beta_{t}+r_{(\beta w)}^{t s} \beta_{t} \otimes w_{s} .
\end{array}
$$

Performing cyclic permutations of the indices $(i j k)$, we have in the end that $(5)$ is equivalent to the following equality:

$$
\begin{gathered}
0=\left(c_{i j}\left(\delta_{k}^{n}-c_{k m} d^{m n}\right)+c_{j k}\left(\delta_{i}^{n}-c_{i m} d^{m n}\right)+c_{k i}\left(\delta_{j}^{n}-c_{j m} d^{m n}\right)\right) \gamma \otimes \alpha_{n} \\
+\left(c_{i j}\left(\delta_{k}^{n}-d^{n m} c_{m k}\right)+c_{j k}\left(\delta_{i}^{n}-d^{n m} c_{m i}\right)+c_{k i}\left(\delta_{j}^{n}-d^{n m} c_{m j}\right)\right) \alpha_{n} \otimes \gamma \\
+r_{(\gamma \beta)}^{s} \gamma \otimes \beta_{s}+r_{(\beta \gamma)}^{s} \beta_{s} \otimes \gamma+r_{(w \alpha)}^{s t} w_{s} \otimes \alpha_{t} \\
\quad+r_{(\alpha w)}^{t s} \alpha_{t} \otimes w_{s}+r_{(w \beta)}^{s t} w_{s} \otimes \beta_{t}+r_{(\beta w)}^{t s} \beta_{t} \otimes w_{s} .
\end{gathered}
$$


Since (8) expresses the vanishing of an element from $A^{2} \otimes A^{1} \oplus A^{1} \otimes A^{2}$, and since $\gamma, w_{1}, \ldots, w_{N}, u_{1}, \ldots, u_{N^{\prime}}$ is a basis in $A^{2}$, we have

$$
\left\{\begin{array}{l}
c_{i j}\left(\delta_{k}^{n}-c_{k m} d^{m n}\right)+c_{j k}\left(\delta_{i}^{n}-c_{i m} d^{m n}\right)+c_{k i}\left(\delta_{j}^{n}-c_{j m} d^{m n}\right)=0, \\
c_{i j}\left(\delta_{k}^{n}-d^{n m} c_{m k}\right)+c_{j k}\left(\delta_{i}^{n}-d^{n m} c_{m i}\right)+c_{k i}\left(\delta_{j}^{n}-d^{n m} c_{m j}\right)=0 .
\end{array}\right.
$$

We shall now show that the first equation of (9) alone implies that $D=C^{-1}$. Fix some arbitrary $n$ and $k, 1 \leq n, k \leq 2 g$, and choose $1 \leq i, j \leq 2 g$ such that $c_{i j}=1, c_{j k}=0$ and $c_{k i}=0$. It is clear that for pair $(i, j)$ we can take $(2 q-1,2 q), 1 \leq q \leq g$, provided $k \neq 2 q-1,2 q$ (such a $q$ always exists since $g \geq 2$ ).

Substituting the chosen $(i, j)$ in the first equation of $(9)$, we have $c_{k m} d^{m n}=\delta_{k}^{n}$, i.e., $D=C^{-1}$.

CASE B. Suppose $a_{1}=\alpha_{1}, a_{2}=\alpha_{2}, a_{3}=\gamma$. In this case, $a_{1} a_{2}=a_{3}, a_{1} a_{3}=a_{2} a_{3}=0$, and (5) can be rewritten as

$$
2 f\left(\alpha_{1}\right) f\left(\alpha_{2}\right) f(\gamma)=f(\gamma) f(\gamma)
$$

Using a direct calculation, we shall now show that (10) does not hold:

$$
\begin{gathered}
f\left(\alpha_{1}\right) f\left(\alpha_{2}\right) f(\gamma)=\left(\alpha_{1} \otimes 1+1 \otimes \alpha_{1}\right)\left(\alpha_{2} \otimes 1+1 \otimes \alpha_{2}\right)(\gamma \otimes 1+1 \otimes \gamma \\
\left.-\sum_{q=1}^{g} \alpha_{2 q-1} \otimes \alpha_{2 q}+\sum_{q=1}^{g} \alpha_{2 q} \otimes \alpha_{2 q-1}+\nu^{s t} \alpha_{s} \otimes \beta_{t}+\mu^{s t} \beta_{s} \otimes \alpha_{t}+\varepsilon^{s t} \beta_{s} \otimes \beta_{t}\right) \\
\quad=\left(\gamma \otimes 1+1 \otimes \gamma+\alpha_{1} \otimes \alpha_{2}-\alpha_{2} \otimes \alpha_{1}\right)(\ldots) \\
\quad=4 \gamma \otimes \gamma+r_{(\gamma w)}^{k} \gamma \otimes w_{k}+r_{(w \gamma)}^{k} w_{k} \otimes \gamma+r_{(w w)}^{s t} w_{s} \otimes w_{t}+R_{1},
\end{gathered}
$$

where $r_{*}^{*} \in \mathbb{Q}$ and $R_{1} \in A^{1} \otimes A^{3} \oplus A^{3} \otimes A^{1}$. Similarly,

$$
\begin{aligned}
f(\gamma) f(\gamma)= & \left(\gamma \otimes 1+1 \otimes \gamma+\sum_{q=1}^{g}\left(-\alpha_{2 q-1} \otimes \alpha_{2 q}+\alpha_{2 q} \otimes \alpha_{2 q-1}\right)\right. \\
& \left.\quad \nu^{s t} \alpha_{s} \otimes \beta_{t}+\mu^{s t} \beta_{s} \otimes \alpha_{t}+\varepsilon^{s t} \beta_{s} \otimes \beta_{t}\right)^{2} \\
= & (\gamma \otimes 1+1 \otimes \gamma)^{2}+\sum_{q=1}^{g}\left(-\alpha_{2 q-1} \otimes \alpha_{2 q}+\alpha_{2 q} \otimes \alpha_{2 q-1}\right)^{2} \\
& +\bar{r}_{(\gamma w)}^{k} \gamma \otimes w_{k}+\bar{r}_{(w \gamma)}^{k} w_{k} \otimes \gamma+\bar{r}_{(w w)}^{s t} w_{s} \otimes w_{t}+R_{2} \\
= & 2 \gamma \otimes \gamma-2 \sum_{q=1}^{g}\left(-\alpha_{2 q-1} \alpha_{2 q} \otimes \alpha_{2 q} \alpha_{2 q-1}\right)+\ldots \\
= & (2-2 g) \gamma \otimes \gamma+\bar{r}_{(\gamma w)}^{k} \gamma \otimes w_{k}+\bar{r}_{(w \gamma)}^{k} w_{k} \otimes \gamma+\bar{r}_{(w w)}^{s t} w_{s} \otimes w_{t}+R_{2},
\end{aligned}
$$

where $\bar{r}_{*}^{*} \in \mathbb{Q}$ and $R_{2} \in A^{1} \otimes A^{3} \oplus A^{3} \otimes A^{1}$.

If identity (10) were true, then, according to our calculations, we would have $8 \gamma \otimes \gamma$ $=(2-2 g) \gamma \otimes \gamma$, whence $g=-3$, which is impossible.

Thus, combining cases $A$ and $B$, we obtain a contradiction under the assumption that there exists a 2-homomorphism $\Delta: A^{*} \rightarrow A^{*} \otimes A^{*}$ satisfying the counit axiom. Hence we have a contradiction under the assumption that $X$ admits a structure of a $2 H$-space. The theorem is proved.

The next result provides a large class of $2 H$-spaces (even $2 H$-groups) with nonabelian fundamental groups. 
Proposition 3.2.5. For any compact acyclic polyhedron $X$, fixed base point $e \in X$, and arbitrary continuous map inv $(X, e) \rightarrow(X, e)$ there exists a 2-valued multiplication $\mu: X \times X \rightarrow \operatorname{Sym}^{2} X$, making $X$ into a $2 H$-group with inverse inv.

For the proof of this result, we need a lemma.

Lemma 3.2.6. For any compact acyclic polyhedron $X$ and any $n \geq 2$, the space $\operatorname{Sym}^{n} X$ is contractible.

Proof. As was shown by A. Dold [19], integral homology $H_{*}\left(\operatorname{Sym}^{n} X ; \mathbb{Z}\right), n \geq 2$, of the $n$-th symmetric power of a cellular space $X$ of finite homological type is uniquely determined by integral homology $H_{*}(X ; \mathbb{Z})$ of $X$ itself (and the number $n$ ). This means that if two spaces $X$ and $Y$ have isomorphic homology $H_{*}(X ; \mathbb{Z}) \cong H_{*}(Y ; \mathbb{Z})$, then $H_{*}\left(\operatorname{Sym}^{n} X ; \mathbb{Z}\right) \cong H_{*}\left(\operatorname{Sym}^{n} Y ; \mathbb{Z}\right) \forall n \geq 2$. Our space $X$ is acyclic, i.e., $H_{*}(X ; \mathbb{Z}) \cong$ $H_{*}(\mathrm{pt} ; \mathbb{Z})$. Therefore, $H_{*}\left(\operatorname{Sym}^{n} X ; \mathbb{Z}\right) \cong H_{*}\left(\operatorname{Sym}^{n} \mathrm{pt} ; \mathbb{Z}\right)=H_{*}(\mathrm{pt} ; \mathbb{Z})$.

Thus, the spaces $\operatorname{Sym}^{n} X, n \geq 2$, are acyclic.

It is not difficult to show that there exists a triangulation of the polyhedron $X^{n}$ such that the canonical action of $S_{n}$ is simplicial, and, therefore, $\operatorname{Sym}^{n} X$ is a polyhedron. Passing to the barycentric subdivision of the chosen triangulation of $X^{n}$, we have that the projection map $f: X^{n} \rightarrow X^{n} / S_{n}=\operatorname{Sym}^{n} X$ is simplicial. Our immediate goal is to show that the map $f_{*}: \pi_{1} X^{n}=\pi_{1} X \times \ldots \times \pi_{1} X \rightarrow \pi_{1}$ Sym $^{n} X$ is an epimorphism.

Fix a vertex $e \in X$. The vertex $[n e] \in \operatorname{Sym}^{n} X$ has a unique preimage $f^{-1}([n e])=$ $(e, \ldots, e) \in X^{n}$. Any loop in $\operatorname{Sym}^{n} X$ starting (and ending) at $[n e]$ is homotopic to a loop $\gamma$, lying in the 1-skeleton of $\operatorname{Sym}^{n} X$. Moreover, $\gamma$ can be chosen to be piecewise linear. It is well known that the projection to the quotient space by an action of a compact group is an open map. Thus, the map $f: X^{n} \rightarrow \operatorname{Sym}^{n} X$ is open, and so is

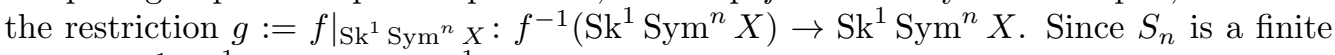
group, $f^{-1}\left(\operatorname{Sk}^{1} \operatorname{Sym}^{n} X\right)=\operatorname{Sk}^{1} X^{n}$.

We now have that $g: \mathrm{Sk}^{1} X^{n} \rightarrow \mathrm{Sk}^{1} \operatorname{Sym}^{n} X$ is an open simplicial map between connected graphs. For such maps, it is not difficult to show that any piecewise linear path in the base lifts (nonuniquely) to a path in the total space. It now follows that any piecewise linear loop in $\mathrm{Sk}^{1} \mathrm{Sym}^{n} X$ starting at $[n e]$ lifts to a path in $\mathrm{Sk}^{1} X^{n}$ which starts and ends at the unique point of the preimage $f^{-1}([n e])=(e, \ldots, e) \in \mathrm{Sk}^{1} X^{n}$. Therefore, for any such loop $\gamma:[0,1] \rightarrow \operatorname{Sk}^{1} \operatorname{Sym}^{n} X, \gamma(0)=\gamma(1)=[n e]$, there is a loop $\widehat{\gamma}:[0,1] \rightarrow \mathrm{Sk}^{1} X^{n}, \widehat{\gamma}(0)=\widehat{\gamma}(1)=(e, \ldots, e)$, such that $\gamma=f \circ \widehat{\gamma}$. But since any element from $\pi_{1} \operatorname{Sym}^{n} X$ can be represented by such a $\gamma$, we have that $f_{*}: \pi_{1} X^{n} \rightarrow \pi_{1} \operatorname{Sym}^{n} X$ is indeed an epimorphism.

Now we want to show that the just proved surjectivity implies that $\pi_{1} \operatorname{Sym}^{n} X$ is trivial. Set $\pi_{1} X=G$. Since $H_{1}(X ; \mathbb{Z})=0$, we have $G^{a b}=G /[G, G]=0$. Since $f\left(x_{\sigma(1)}, \ldots, x_{\sigma(n)}\right)=f\left(x_{1}, \ldots, x_{n}\right) \forall x_{1}, \ldots, x_{n} \in X, \forall \sigma \in S_{n}$, we have $f_{*}\left(g_{\sigma(1)}, \ldots, g_{\sigma(n)}\right)$ $=f_{*}\left(g_{1}, \ldots, g_{n}\right) \forall g_{1}, \ldots, g_{n} \in G, \forall \sigma \in S_{n}$, where $f_{*}: G \times \ldots \times G \rightarrow \pi_{1} \operatorname{Sym}^{n} X$ is the above epimorphism of fundamental groups. We also have

$$
\begin{aligned}
f_{*}\left(g_{1}, \ldots, g_{n}\right) & =f_{*}\left(\left(g_{1}, e, \ldots, e\right) \cdot\left(e, g_{2}, \ldots, e\right) \ldots\left(e, e, \ldots, g_{n}\right)\right) \\
& =f_{*}\left(g_{1}, e, \ldots, e\right) f_{*}\left(e, g_{2}, \ldots, e\right) \ldots f_{*}\left(e, e, \ldots, g_{n}\right) \\
& =f_{*}\left(g_{1}, e, \ldots, e\right) f_{*}\left(g_{2}, e, \ldots, e\right) \ldots f_{*}\left(g_{n}, e, \ldots, e\right) \\
& =f_{*}\left(g_{1} g_{2} \ldots g_{n}, e, \ldots, e\right) \quad \forall g_{1}, \ldots, g_{n} \in G .
\end{aligned}
$$

Thus, already the restriction

$$
\bar{f}_{*}=\left.f_{*}\right|_{G \times\{e\} \times \ldots \times\{e\}}: G \times\{e\} \times \ldots \times\{e\} \rightarrow \pi_{1} \operatorname{Sym}^{n} X
$$

is an epimorphism. Moreover,

$$
f_{*}\left(g_{1} g_{2}, e, \ldots, e\right)=f_{*}\left(g_{1}, g_{2}, e, \ldots, e\right)=f_{*}\left(g_{2}, g_{1}, e, \ldots, e\right)=f_{*}\left(g_{2} g_{1}, e, \ldots, e\right) .
$$


Therefore, the epimorphism $\bar{f}_{*}: G \rightarrow \pi_{1} \operatorname{Sym}^{n} X$ has the property that $\bar{f}_{*}\left(g_{1} g_{2}\right)=$ $\bar{f}_{*}\left(g_{2} g_{1}\right)$, i.e., $\bar{f}_{*}\left(g_{1} g_{2} g_{1}^{-1} g_{2}^{-1}\right)=e \forall g_{1}, g_{2} \in G$. This means that the epimorphism $\bar{f}_{*}: G \rightarrow \pi_{1} \operatorname{Sym}^{n} X$ maps the entire commutator subgroup $[G, G]$ to the identity. But $[G, G]=G$, and therefore $\pi_{1} \operatorname{Sym}^{n} X$ is trivial.

We already know that the polyhedron $\operatorname{Sym}^{n} X$ is simply connected and acyclic. But that, as is known from the homotopy theory of cellular spaces, implies contractibility. The lemma is proved.

Proof of Proposition 3.2.5. We are given a compact acyclic polyhedron. By the just proved lemma, $\operatorname{Sym}^{2} X$ is a contractible space. Consider the constant map $\mu_{0}: X \times X \rightarrow$ $\operatorname{Sym}^{2} X, \mu_{0}(x, y)=[2 e]$ and its restriction to the wedge product $\bar{\mu}_{0}: X \vee X \rightarrow$ Sym $^{2} X$. Since $\operatorname{Sym}^{2} X$ is contractible, $\bar{\mu}_{0}$ can be connected by a homotopy $\bar{\mu}_{t}$ with any other map from $X \vee X$ to $\operatorname{Sym}^{2} X$, in particular, with $\bar{\mu}_{1}: X \vee X \rightarrow \operatorname{Sym}^{2} X, \bar{\mu}_{1}(x, e)=$ $\bar{\mu}_{1}(e, x)=[2 x] \forall x \in X$. The pair $(X \times X, X \vee X)$, being cellular, is a Borsuk pair, and therefore the homotopy $\bar{\mu}_{t}:(X \vee X) \times I \rightarrow \operatorname{Sym}^{2} X$ can be extended to a homotopy $\mu_{t}:(X \times X) \times I \rightarrow \operatorname{Sym}^{2} X$. In particular, we obtain a map $\mu:=\mu_{1}: X \times X \rightarrow \operatorname{Sym}^{2} X$ such that $\mu(x, e)=\mu(e, x)=[2 x] \forall x \in X$. Thus, we have a 2-valued multiplication $\mu$ with unit on $X$, making $X$ a $2 H$-space. The homotopy associativity of $\mu$ follows trivially from the fact that the space $\operatorname{Sym}^{4} X$ is also contractible.

Finally, we want to show that an arbitrary map inv: $X \rightarrow X$ is a homotopy inverse. Indeed, the map

$$
X \stackrel{\Delta}{\longrightarrow} X \times X \stackrel{\mathrm{id} \times \mathrm{inv}}{\longrightarrow} X \times X \stackrel{\mu}{\longrightarrow} \operatorname{Sym}^{2} X, \quad x \mapsto \mu(x, \operatorname{inv}(x)),
$$

as a map into a contractible space, is homotopic to any other map $X \rightarrow \operatorname{Sym}^{2} X$, and, in particular, to any map of the form $x \mapsto[e, h(x)]$, where $h: X \rightarrow X$ is an arbitrary map. The same is true for the map

$$
X \stackrel{\Delta}{\longrightarrow} X \times X \stackrel{\text { inv } \times \mathrm{id}}{\longrightarrow} X \times X \stackrel{\mu}{\longrightarrow} \mathrm{Sym}^{2} X .
$$

Therefore, inv is a homotopy inverse. Thus, all axioms of a $2 H$-group on $X$ are satisfied, and the proposition is proved.

Proposition 3.2.5 gives us a class of $2 H$-spaces ( $2 H$-groups) with nonabelian (even perfect) fundamental groups. In particular, this class includes all punctured homology spheres (for example, the punctured Poincaré sphere). This class is also closed under wedge products and direct products.

\section{ACKNOWLEDGMENTS}

The author is deeply grateful to Professor V. M. Buchstaber for posing the problems, and for his constant attention to and interest in this work. The author thanks Professors A. V. Zarelua, T. E. Panov, V. A. Smirnov, A. V. Chernavsky, E. V. Shchepin, Associated Professor I. V. Arzhantsev, and Senior Researcher S. A. Melikhov for useful discussions. The author is also grateful to the entire faculty of Geometry and Topology at the Mechanics and Mathematics Department of Moscow University for their support and attention.

\section{REFERENCES}

[1] J. W. Alexander, Note on Riemann spaces, Bull. Amer. Math. Soc. 26 (1920), no. 8, $370-372$. MR. 1560318

[2] I. Berstein and A. L. Edmonds, The degree and branch set of a branched covering, Invent. Math. 45 (1978), 213-220. MR0474261 (57:13908)

[3] G. E. Bredon, Introduction to compact transformation groups, Academic Press, New York-London, 1972. MR 0413144 (54:1265) 
[4] G. E. Bredon, Sheaf theory, McGraw-Hill Book Co., New York-Toronto-London, 1967. MR.0221500 $(36: 4552)$

[5] V. M. Buchstaber, n-valued groups: theory and applications, Mosc. Math. J. 6 (2006), no. 1, 57-84. MR2265947 (2007i:20102)

[6] V. M. Buchstaber, Functional equations that are associated with addition theorems for elliptic functions, and two-valued algebraic groups, Uspekhi Mat. Nauk 45 (1990), no. 3(273), 185-186; English transl., Russian Math. Surveys 45 (1990), no. 3, 213-215. MR1071939 (91j:39002)

[7] V. M. Buchstaber and S. P. Novikov, Formal groups, power systems and Adams operators, Mat. Sb. (N.S.) 84(126) (1971), 81-118. (Russian) MR0291159 (45:253)

[8] V. M. Buchstaber and E. G. Rees, Frobenius n-homomorphisms, transfers and branched coverings, Math. Proc. Cambridge Philos. Soc. 144 (2008), no. 1, 1-12. MR2388228(2009b:55017)

[9] V. M. Buchstaber and E. G. Rees, Rings of continuous functions, symmetric products, and Frobenius algebras, Uspekhi Mat. Nauk 59 (2004), no. 1(355), 125-144; English transl., Russian Math. Surveys 59 (2004), no. 1, 125-145. MR2069166 (2005f:54031)

[10] V. M. Buchstaber and E. G. Rees, The Gelfand map and symmetric products, Selecta Math. (N.S.) 8 (2002), no. 4, 523-535. MR.1951205 (2003k:13009)

[11] V. M. Buchstaber and E. G. Rees, A constructive proof of the generalized Gelfand isomorphism, Funktsional. Anal. i Prilozhen. 35 (2001), no. 4, 20-25; English transl., Funct. Anal. Appl. 35 (2001), no. 4, 257-260. MR.1879115 (2002h:46081)

[12] V. M. Buchstaber and E. G. Rees, Multivalued groups, n-Hopf algebras and n-ring homomorphisms, Lie groups and Lie algebras, Math. Appl., 433. Kluwer Acad. Publ., Dordrecht, 1998, 85-107. MR.1628811 (99f:20111)

[13] V. M. Buchstaber and E. G. Rees, Multivalued groups, their representations and Hopf algebras, Transform. Groups 2 (1997), no. 4, 325-349. MR1486035 (99b:20071)

[14] V. M. Buchstaber and E. G. Rees, Multivalued groups and Hopf n-algebras, Uspekhi Mat. Nauk 51 (1996), no. 4(310), 149-150; English transl., Russian Math. Surveys 51 (1996), no. 4, 727-729. MR.1422232

[15] V. M. Buchstaber and A. P. Veselov, Integrable correspondences and algebraic representations of multivalued groups, Internat. Math. Res. Notices 8 (1996), 381-400. MR1393330 (98f:58098)

[16] A. V. Chernavsky, Finite-to-one open mappings of manifolds. Mat. Sb. (N.S.) 65(107) (1964), 357-369. (Russian) MR0172256 (30:2476)

[17] A. Dold, Ramified coverings, orbit projections and symmetric powers, Math. Proc. Cambridge Philos. Soc. 99 (1986), no. 1, 65-72. MR809499 (88h:55018)

[18] A. Dold, Lectures on algebraic topology, Die Grundlehren der mathematischen Wissenschaften, Band 200, Springer-Verlag, New York-Berlin, 1976. (German) MR0415602 (54:3685)

[19] A. Dold, Homology of symmetric products and other functors of complexes, Ann. of Math. (2) 68 (1958), 54-80. MR0097057 (20:3537)

[20] V. Dragovic, Geometrization and generalization of the Kowalevski top, Comm. Math. Phys. 298 (2010), no. 1, 37-64. MR2657814 (2011g:37155)

[21] R. Engelking, General topology, Mathematical Monographs, Vol. 60, PWN-Polish Scientific Publishers, Warsaw, 1977. MR0500780(58:18316b)

[22] G. Frobenius, Über Gruppencharaktere, Sitzungsber. Preuß. Akad. Wiss. Berlin 1896, 985-1021.

[23] G. Frobenius, Über die Primfaktoren der Gruppendeterminante, Sitzungsber. Preuß. Akad. Wiss. Berlin 1896, 1343-1382.

[24] D. V. Gugnin, Polynomially dependent homomorphisms and Frobenius n-homomorphisms, Tr. Mat. Inst. Steklova 266 (2009), 64-96; English transl., Proc. Steklov Inst. Math. 266 (2009), no. 1, 59-90. MR2603261 (2011e:16077)

[25] K. Kuratowski, Topology, Vol. I. Academic Press, New York-London, 966. MR0217751 (36:840)

[26] R. Narasimhan, Introduction to the theory of analytic spaces, Lecture Notes in Mathematics, No. 25, Springer-Verlag, Berlin-New York, 1966. MR0217337 (36:428)

[27] T. E. Panov, On the structure of the 2-Hopf algebra in the cohomology of four-dimensional manifolds. Uspekhi Mat. Nauk 51 (1996), no. 1(307), 161-162; English transl., Russian Math. Surveys 51 (1996), no. 1, 155-157. MR1392685 (97f:57043)

[28] L. Smith, Transfer and ramified coverings, Math. Proc. Cambridge Philos. Soc. 93 (1983), $485-493$. MR698352(85f:57002)

Mechanics and Mathematics Department, Moscow State University, Moscow 11991, Russia E-mail address: dmitry-gugnin@yandex.ru 